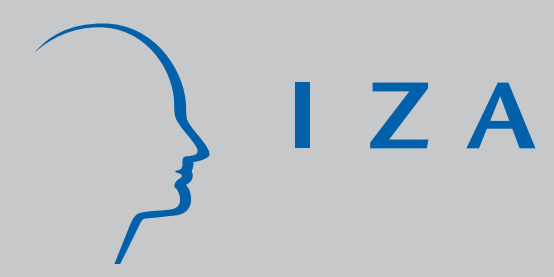

IZA DP No. 843

Simulation and Estimation of Hedonic Models

J ames J. Heckman

Rosa Matzkin

Lars Nesheim

August 2003 


\title{
Simulation and Estimation of Hedonic Models
}

\author{
James J. Heckman \\ University of Chicago, American Bar Foundation \\ and IZA Bonn \\ Rosa Matzkin \\ Northwestern University \\ Lars Nesheim \\ Institute for Fiscal Studies, University College London
}

Discussion Paper No. 843
August 2003

IZA

P.O. Box 7240

D-53072 Bonn

Germany

Tel.: +49-228-3894-0

Fax: +49-228-3894-210

Email: iza@iza.org

This Discussion Paper is issued within the framework of IZA's research area Evaluation of Labor Market Policies and Projects. Any opinions expressed here are those of the author(s) and not those of the institute. Research disseminated by IZA may include views on policy, but the institute itself takes no institutional policy positions.

The Institute for the Study of Labor (IZA) in Bonn is a local and virtual international research center and a place of communication between science, politics and business. IZA is an independent, nonprofit limited liability company (Gesellschaft mit beschränkter Haftung) supported by Deutsche Post World Net. The center is associated with the University of Bonn and offers a stimulating research environment through its research networks, research support, and visitors and doctoral programs. IZA engages in (i) original and internationally competitive research in all fields of labor economics, (ii) development of policy concepts, and (iii) dissemination of research results and concepts to the interested public. The current research program deals with (1) mobility and flexibility of labor, (2) internationalization of labor markets, (3) welfare state and labor market, (4) labor markets in transition countries, (5) the future of labor, (6) evaluation of labor market policies and projects and (7) general labor economics.

IZA Discussion Papers often represent preliminary work and are circulated to encourage discussion. Citation of such a paper should account for its provisional character. A revised version may be available on the IZA website (www.iza.org) or directly from the author. 
IZA Discussion Paper No. 843

August 2003

\section{ABSTRACT}

\section{Simulation and Estimation of Hedonic Models*}

Making use of restrictions imposed by equilibrium, theoretical progress has been made on the nonparametric and semiparametric estimation and identification of scalar additive hedonic models (Ekeland, Heckman, and Nesheim, 2002) and scalar nonadditive hedonic models (Heckman, Matzkin, and Nesheim, 2002). However, little is known about the practical aspects of estimating such models or of the characteristics of equilibrium in such models. This paper presents computational and analytical results that fill some of these gaps. We simulate and estimate examples of equilibrium in the additive hedonic models and provide evidence on the performance of several estimation techniques. We also simulate examples of equilibria in nonadditive models and provide evidence on the performance of the nonadditive estimation techniques developed in Heckman, Matzkin, and Nesheim (2002).

JEL Classification: C31

Keywords: hedonic models, nonparametric methods, simulation, Monte Carlo, additive models, nonadditive models

Corresponding author:

James J. Heckman

Department of Economics

University of Chicago

1126 East $59^{\text {th }}$ Street

Chicago, IL 60637

USA

Tel.: +1 7737020634

Fax: +1 7737028490

Email: jjh@uchicago.edu

* This research was supported by NSF-00-99195 to James J. Heckman. We would like to thank Andrew Chesher, Hidehiko Ichimura and Simon Lee for helpful comments. 


\section{Introduction}

Hedonic models are general equilibrium frameworks that characterize the pricing of differentiated goods, viewed as bundles of attributes, and the demand and supply of those goods (attributes) under different assumptions about preferences and technology. They allow for a systematic economic analysis of the demand and supply of quality. Quality includes enhancement of the attributes of a good embodied in a unit of the good (such as the attributes of a house or a car as in Rosen, 1974), characteristics of a job (risk or unpleasantness as in Tinbergen, 1956, Sattinger 1975, 1980, 1993, and in Thaler and Rosen, 1975) or the amenities offered by an environmental or recreational improvement (as in Smith and Huang, 1995 and Banzhaf, Sieg, Smith, and Walsh, 2000). Understanding the structure of demand in markets for differentiated products is a crucial ingredient of models of monopoly pricing (Wilson, 1993; Rochet and Stole, 2001; and Armstrong, 1996). The hedonic model underlies general equilibrium analyses of local public goods (Epple, 1987; Epple and Sieg, 1999; and Bayer, 2000) and models in which social interactions are priced (Nesheim, 2001). The promise of the hedonic approach is great. It offers insight into the economics of variety and hetero-

geneity in product quality and worker skill which are hallmark features of 
modern economies. It offers a consistent approach to adjusting price indices for quality and allowing for valuation of new goods (or environmental offerings) that can be viewed as new packages of old attributes (Lancaster, 1966, 1975; Triplett, 2000; or the essays in Bresnahan and Gordon, 1997). With hedonic models, it is possible to interpret wage data on heterogeneous labor, to evaluate alternative policy proposals for workplace safety (as in Kniesner and Leeth, 1988, 1995), to evaluate proposals to subsidize education and job training (Teulings and van Rens, 2002; and Tinbergen, 1956), and to examine their consequences for worker and firm welfare and for wage inequality.

The potential applications of hedonic models are myriad but their application and development, except in certain special cases, have been hindered by computational difficulties, approximations that ignore the implications of equilibrium in the hedonic model, and the widely held belief that identification of the structural parameters in a hedonic model is not possible using data from a single market. Recent theoretical progress has been made in understanding these issues making use of restrictions imposed by equilibrium. Ekeland, Heckman, and Nesheim (2002) and Heckman, Matzkin, and Nesheim (2002) have shown that contrary to the widely held belief that identification is impossible in a single market, nonparametric and semipara- 
metric estimation and identification of scalar additive hedonic models (Ekeland, Heckman, and Nesheim, 2002) and scalar nonadditive hedonic models (Heckman, Matzkin, and Nesheim, 2003) is possible.

However, little is known about the practical aspects of estimating such models or of the characteristics of equilibrium in these two classes of models. This paper presents computational and analytical results that fill these gaps. We simulate and estimate examples of equilibrium in these classes of hedonic models and provide evidence on the performance of several estimation techniques. The simulations show the shapes of the pricing function that result from various assumptions about the underlying structural parameters in the economy. The estimation results demonstrate that structural parameters in an additive economy can be precisely estimated. In addition, these results demonstrate that in a nonadditive economy nonparametric techniques can be used to recover estimates of the structural parameters.

In section 2 we present the general hedonic model. In section 4 we discuss identification of the model and conditions sufficient for identification in the additive and nonadditive cases. In section 5 we discuss estimation of the model based on the earlier results. In section 6 we present simulation and estimation results before concluding in section 7 . 


\section{General Hedonic Model}

We first present a general statement of the classical hedonic model. For specificity, consider a labor market setting. Our analysis applies more generally, but it is useful to have a specific example in hand. Assume a static model. Consumers (workers) match to single worker firms. Workers are heterogeneous. They have characteristics $(x, \varepsilon)$ where $x \in X \subseteq R^{n_{x}}$ and $\varepsilon \in E \subseteq R^{n_{\varepsilon}}$ are observable and unobservable (to the econometrician) characteristics that affect their utility from different job types. Firms are also heterogeneous. They have characteristics $(y, \eta)$ where $y \in Y \subseteq R^{n_{y}}$ and $\eta \in H \subseteq R^{n_{\eta}}$ are observable and unobservable characteristics that affect the output and profits they obtain from different job types. Job types have characteristics $z \in Z$ where $Z \subseteq R^{n_{z}}$. $Z$ is the set of feasible job types. For example, $z$ could be a (possibly multidimensional) measure of the riskiness of the job. Alternatively, in a housing market setting, $z$ could be a vector of characteristics of a neighborhood or a house. We focus attention on the classical case where $n_{z}=n_{\varepsilon}=n_{\eta}$ assuming a smooth equilibrium pricing function. This is the hedonic model analyzed in Tinbergen (1956), Rosen (1974), Epple (1987) and Kniesner and Leeth $(1988,1995)$. It is also the example that has dominated much of the literature on hedonic models. The theoretical analysis, 
the simulation models, and the empirical results to follow in sections 4 and 6 restrict the analysis further and focus on the scalar hedonic model in which $n_{z}=1$.

The distribution of consumer characteristics in the population is characterized by the density functions $f_{x}$ and $f_{\varepsilon}$ both strictly greater than zero in the interiors of $X$ and $E$ respectively. We assume $x$ is independent of $\varepsilon$. Similarly, the distribution of firm characteristics are characterized by the density functions $f_{y}$ and $f_{\eta}$ also strictly positive in the interiors of their respective supports. $y$ is independent of $\varepsilon$.

Workers of type $(x, \varepsilon)$ choose jobs of type $z$ to maximize utility. $P(z)$ is the earnings of workers supplying attribute vector $z$, which is a disamenity. To focus on the main ideas, we study the quasilinear utility model (also known as the transferable utility model in the assignment literature and in the theoretical public economics literature. See Gretsky, Ostroy, and Zame, 1999 and Wooders, 1994). Define $U^{*}(c, z, x, \varepsilon)=c-U(z, x, \varepsilon)$ where $x$ and $\varepsilon$ are as defined above and $c$ is consumption. For simplicity assume $c=P(z)$, so workers consume their earnings. More generally, $c=P(z)+R$ where $R$ is non-labor income, but for ease of exposition, assume $R=0$. Workers who don't work get reservation utility $V_{0}$. We initially restrict our analysis 
to economies for which the equilibrium price function is smooth. Similar analyses can be done for economies in which the equilibrium price function is not smooth. Smoothness is not a generic property of hedonic models, even when the underlying preferences are smooth. ${ }^{1}$ Given $P(z)$, a twice continuously differentiable price function, and assuming the utility function is twice differentiable, for those who choose to work we obtain the following first order conditions for a maximum

$$
P_{z}(z)-U_{z}(z, x, \varepsilon)=0 .
$$

The second order conditions (SOC) require that $P_{z z^{\prime}}-U_{z z^{\prime}}$ be negative definite. Assuming the SOC are satisfied and using the Implicit Function Theorem, (1) determines $z=s(x, \varepsilon)$, the quality of the good supplied by each worker $(x, \varepsilon)$. Assuming $U_{z \varepsilon^{\prime}}$ is invertible it also determines the inverse mapping $\varepsilon=\widetilde{s}(z, x)$. Note that $\widetilde{s}(z, x)$ implicitly depends on the marginal price function $P_{z}(z)$. The two mappings $s(x, \varepsilon)$ and $\widetilde{s}(z, x)$ are the focus of both our theoretical and empirical study of the classical hedonic model.

Firms of type $(y, \eta)$ demand attribute $z$ and maximize profits which are

\footnotetext{
${ }^{1}$ For examples of sorting problems with non-smooth pricing functions see Wilson (1993), Nesheim (2001), and Heckman, Matzkin, and Nesheim (2002).
} 
equal to output $\Gamma(z, y, \eta)$ minus production $\operatorname{costs} P(z)$ where $y$ and $\eta$ are defined above. We assume that the production function is twice differentiable. If the firm hires no workers, reservation profits are $\Pi_{0}$. Otherwise, profits are $\Pi(z, y, \eta, P(z))=\Gamma(z, y, \eta)-P(z)$ and the first order conditions for a maximum of each firm that enters the market are

$$
\Gamma_{z}(z, y, \eta)-P_{z}(z)=0
$$

The second order conditions require that $\Gamma_{z z^{\prime}}-P_{z z^{\prime}}$ be negative definite. Assuming the SOC are satisfied and using the Implicit Function Theorem, (2) defines $z=d(y, \eta)$, the type of job demanded by each firm $(y, \eta)$. Assuming $\Gamma_{z \varepsilon^{\prime}}$ is invertible, it also determines the inverse mapping $\eta=\widetilde{d}(z, y)$. As on the supply side, $\widetilde{d}(z, y)$ implicitly depends on the marginal price function $P_{z}(z)$

In equilibrium, the density of the demanded $z$ must equal the density of the supplied $z$ for all values of $z$. To express this condition in terms of the primitive functions, consider the transformation defined by the consumer first order conditions $\varepsilon=\widetilde{s}(z, x)$ and $x=x$. The Jacobian of this transformation is $\operatorname{det}\left(\begin{array}{cc}\frac{\partial \widetilde{s}(z, x)}{\partial z} & \frac{\partial \widetilde{s}(z, x)}{\partial x} \\ 0 & 1\end{array}\right)=\operatorname{det}\left(\frac{\partial \widetilde{s}(z, x)}{\partial z}\right)$. Assuming that all potential workers 
actually work, this transformation induces a density of consumers supplying each type of job z. Thus, the Supply Density is

$$
\int_{X} f_{\varepsilon}(\widetilde{s}(z, x)) f_{x}(x) \operatorname{det}\left(\frac{\partial \widetilde{s}(z, x)}{\partial z}\right) d x
$$

Analogous arguments produce the density of $z$ demanded. Consider the transformation derived from the firms' FOC $\eta=\widetilde{d}(z, y)$ and $y=y$ with Jacobian $\operatorname{det}\left(\begin{array}{cc}\frac{\partial \widetilde{d}(z, y)}{\partial z} & \frac{\partial \widetilde{d}(z, y)}{d y} \\ 0 & 1\end{array}\right)=\operatorname{det}\left(\frac{\partial \widetilde{d}(z, y)}{\partial z}\right)$. Assuming that all firms enter the market, this transformation induces a density of demand for every job type z. The Demand Density is:

$$
\int_{Y} f_{\eta}(\widetilde{d}(z, y)) f_{y}(y) \operatorname{det}\left(\frac{\partial \widetilde{d}(z, y)}{\partial z}\right) d y
$$

Equilibrium in hedonic markets requires that demand and supply be equated at each point of the support of $z$. So, equilibrium prices must satisfy the following second order differential equation in $P(z)$

$$
\begin{aligned}
& \int_{X} f_{\varepsilon}(\widetilde{s}(z, x)) f_{x}(x) \operatorname{det}\left(\frac{\partial \widetilde{s}(z, x)}{\partial z}\right) d x= \\
& \int_{Y} f_{\eta}(\widetilde{d}(z, y)) f_{y}(y) \operatorname{det}\left(\frac{\partial \widetilde{d}(z, y)}{\partial z}\right) .
\end{aligned}
$$


Observe that $\widetilde{s}(z, x)$ and $\widetilde{d}(z, y)$ implicitly depend on $P_{z}$ and $P_{z z^{\prime}}$. In addition, the solution depends on the structural parameters of the model: technology of the firms $\Gamma$, the utility function $U$ of the workers, and the distributions of firms and workers in the population $\left(f_{x}, f_{\varepsilon}, f_{y}, f_{\eta}\right)$. Economic theory implies that marginal products and marginal utilities are nonnegative in most cases. In order for agents to participate in the market, firms and workers must receive wages and profits above reservation levels. If not, equation (3) must be suitably adjusted. These criteria generate the boundary conditions that determine the solution of the differential equation for equilibrium prices.

Equations (1), (2), and (3) and the data generated by them are the focus of our analysis. They determine all the theoretical and statistical properties of the model. In general, equations like (3) have no closed form solution, except for the special case of the Tinbergen model, which is developed below that is not identified in a single cross section. While progress has been made in understanding such equations in the mathematics and numerical analysis literature little is known about their solutions in economics. ${ }^{2}$ The inability to compute or even characterize the equilibria from this model even in the

\footnotetext{
${ }^{2}$ Notable exceptions include Kniesner and Leeth (1995), Teulings (1995), and Nesheim (2001).
} 
cases of a scalar attribute has inhibited application of the hedonic model to economic problems. It has also hindered understanding of the statistical properties of hedonic models relevant to identification and estimation. Our simulations presented in section 6 help remedy this problem. We first present the Tinbergen model which implicitly or explicitly has been the point of departure for all empirical work on hedonics.

\section{Tinbergen's Linear-Quadratic Model}

Assume preferences are quadratic in $z$ and linear in $c$, unearned income $R=$ 0 , and that individual heterogeneity $(x, \varepsilon)$ only affects utility through the single index $\theta=\mu_{\theta}(x)+\varepsilon$ where $\operatorname{dim}(\theta)=\operatorname{dim}(z)^{3}$. Consumers maximize

$$
U(c, z, \theta, A)=P(z)+\theta^{\prime} z-\frac{1}{2} z^{\prime} A z
$$

The conditions determining a consumer maximum are

$$
P_{z}+\theta-A z=0
$$

\footnotetext{
${ }^{3}$ The model in this example was first analyzed by Tinbergen (1956) and has been used by Epple (1987) and Tauchen and Witte (2001) among others.
} 
where $P_{z z^{\prime}}-A$ is negative definite. On the firm side, assume the production function is quadratic in $z$ and that firm heterogeneity only affects profits through the single index $\nu=\mu_{\nu}(y)+\eta$ where $\operatorname{dim}(\nu)=\operatorname{dim}(z)$. Profits are

$$
\Pi(z, \nu, B, P(z))=\nu^{\prime} z-\frac{1}{2} z^{\prime} B z-P(z)
$$

and the conditions determining a firm's optimum are

$$
\nu-B z-P_{z}=0
$$

where $-\left(B+P_{z z^{\prime}}\right)$ is negative definite. The distributions of $\theta$ and $\nu$ in the population are normal. The distribution of $\theta$ is $\theta \sim N\left(\mu_{\theta}, \Sigma_{\theta}\right)$, and the distribution of $\nu$ is $\nu \sim N\left(\mu_{\nu}, \Sigma_{\nu}\right)$.

An arbitrary price function induces a density of demand and a density of supply at every location $z$. The equilibrium price function can be found by equating these densities at every point $z$ and solving the differential equation (3). However, in the linear-quadratic-normal case one can correctly guess that the solution to the problem is quadratic in $z$

$$
P(z)=\pi_{0}+\pi_{1}^{\prime} z+\frac{1}{2} z^{\prime} \pi_{2} z
$$


and then find the coefficients $\left(\pi_{0}, \pi_{1}, \pi_{2}\right)$ that satisfy the equilibrium equation. Assuming the price function is quadratic, the first order condition for a consumer is

$$
\pi_{1}+\pi_{2} z+\theta-A z=0 .
$$

For a firm, it is

$$
\nu-B z-\pi_{1}-\pi_{2} z=0 .
$$

The second order conditions require that both $A-\pi_{2}$ and $B+\pi_{2}$ are positive definite. Thus we may solve for $z$ from (4) to obtain

$$
z=\left(A-\pi_{2}\right)^{-1}\left(\theta+\pi_{1}\right)
$$

and from (5) to obtain

$$
z=\left(B+\pi_{2}\right)^{-1}\left(\nu-\pi_{1}\right) .
$$

These equations define mappings from workers $\theta$ and firms $\nu$ to job types $z$. These mappings determine the density of supply and demand at every location and the types of workers and firms at every location. Equilibrium is characterized by a vector $\pi_{1}$ and a matrix $\pi_{2}$ that equate demand and 
supply at all $z$. However, since both $\theta$ and $\nu$ are normally distributed, this only requires equating the mean and variance of supply and demand.

The mean supply $E^{S}(z)$ is obtained from (4):

$$
\text { (Average Supply) } \quad E^{S}(z)=\left(A-\pi_{2}\right)^{-1} E\left(\theta+\pi_{1}\right)
$$

The mean demand is obtained from (7):

$$
\text { (Average Demand) } \quad E^{D}(z)=\left(B+\pi_{2}\right)^{-1} E\left(\nu-\pi_{1}\right)
$$

Since $\mu_{\theta}=E(\theta)$ and $\mu_{\nu}=E(\nu)$, the condition $E^{S}(z)=E^{D}(z)$ implies that

$$
\text { (Equality of means) }\left(A-\pi_{2}\right)^{-1}\left(\mu_{\theta}+\pi_{1}\right)=\left(B+\pi_{2}\right)^{-1}\left(\mu_{\nu}-\pi_{1}\right) .
$$

Rearranging terms, we obtain an explicit expression for $\pi_{1}$ in terms of $A, B, \mu_{\theta}, \mu_{\nu}$ and $\pi_{2}$ :

$$
\pi_{1}=\left[\left(A-\pi_{2}\right)^{-1}+\left(B+\pi_{2}\right)^{-1}\right]^{-1}\left[-\left(A-\pi_{2}\right)^{-1} \mu_{\theta}+\left(B+\pi_{2}\right)^{-1} \mu_{\nu}\right] .
$$

To determine $\pi_{2}$, compute the variances of supply and demand from (4) 
and (7) respectively to obtain:

$$
\begin{aligned}
& \Sigma_{z}^{S}=\left(A-\pi_{2}\right)^{-1} \Sigma_{\theta}\left(A-\pi_{2}\right)^{-1} \\
& \Sigma_{z}^{D}=\left(B+\pi_{2}\right)^{-1} \Sigma_{\nu}\left(B+\pi_{2}\right)^{-1}
\end{aligned}
$$

where $\Sigma_{z}^{S}$ is the variance of supply and $\Sigma_{z}^{D}$ is the variance of demand. From equality of variances of the demand and supply distributions we obtain an implicit equation for $\pi_{2}$ :

(Equality of variances) $\quad\left(A-\pi_{2}\right)^{-1} \Sigma_{\theta}\left(A-\pi_{2}\right)^{-1}=\left(B+\pi_{2}\right)^{-1} \Sigma_{\nu}\left(B+\pi_{2}\right)^{-1}$.

We pin down initial conditions using the restrictions that $U \geq \bar{U}$, a reservation value, and profits are positive $(\Pi \geq 0)$. Equilibrium profits as a function of location are $\frac{1}{2} z^{\prime}\left(B+\pi_{2}\right) z-\pi_{0}$. Hence nonnegativity of profits implies $-\pi_{0} \geq 0$ since $\left(B+\pi_{2}\right)$ is positive definite by the second order conditions. Setting reservation utility equal to zero, a similar argument on the worker side implies $\pi_{0} \geq 0$. Hence $\pi_{0}=0$.

Once we have solved for $\pi_{1}$ and $\pi_{2}$, (4) and (7) also define the equilibrium matching function linking the characteristics of suppliers $(\theta)$ to those 
of demanders $(\nu)$. For each $z$, this function is

$$
\left(A-\pi_{2}\right)^{-1}\left(\theta+\pi_{1}\right)=\left(B+\pi_{2}\right)^{-1}\left(\nu-\pi_{1}\right) .
$$

Thus, the equilibrium relationship between $\theta$ and $\nu$ is

$$
\theta=\left(A-\pi_{2}\right)\left(B+\pi_{2}\right)^{-1}\left(\nu-\pi_{1}\right)-\pi_{1} .
$$

This relationship has important empirical implications as noted by Epple (1987) and Kahn and Lang (1988). Conditional on location choice, worker and firm characteristics are not statistically independent in equilibrium. There is a functional relationship between them.

In the separable case where $\Sigma_{\theta}, \Sigma_{\nu}, A$, and $B$ are diagonal, $\pi_{2}$ is diagonal. Effectively, this is a scalar case. In the scalar case, equality of variances implies that $\left(A-\pi_{2}\right)^{2} \Sigma_{\nu}=\left(B+\pi_{2}\right)^{2} \Sigma_{\theta}$. Since the second order conditions imply that $A-\pi_{2}>0$ and $B+\pi_{2}>0$. Defining $\sigma_{\theta}=\left(\Sigma_{\theta}\right)^{\frac{1}{2}}$ and $\sigma_{\nu}=\left(\Sigma_{\nu}\right)^{\frac{1}{2}}$, this means that ${ }^{4}$

$$
\pi_{2}=\frac{A \sigma_{\nu}-B \sigma_{\theta}}{\sigma_{\theta}+\sigma_{\nu}}
$$

\footnotetext{
${ }^{4}$ The other root violates second order conditions.
} 


$$
\pi_{1}=\frac{-\mu_{\theta} \sigma_{\nu}+\mu_{\nu} \sigma_{\theta}}{\sigma_{\theta}+\sigma_{\nu}}
$$

$\pi_{2}$, the curvature of the price function, is a weighted average of the curvatures of workers' and firms' preference and technology functions. $\pi_{1}$ is a weighted average of the means of worker and firm distributions of heterogenity. In both cases, the weights depend on the relative variances of worker and firm heterogeneity. If workers are much more heterogeneous than firms $\sigma_{\theta}>>\sigma_{\nu}$, $\pi_{2}$ will approximately equal $B$, the curvature of firms' technology. If $\sigma_{\theta}=\sigma_{\nu}$ and $A=B, \pi_{2}=0$ is a solution and the equilibrium price function is linear in $z$. If $\sigma_{\theta}=\sigma_{\nu}$, but $A \neq B$, then $\pi_{2}=\frac{A-B}{2}$. In the polar cases when $\sigma_{\theta}=0$ or $\sigma_{\nu}=0$ then there is effectively only one type of consumer or one type of firm respectively. If $\sigma_{\theta}=0$ and $\sigma_{\nu}>0$, then $\pi_{2}=A$ and $\pi_{1}=-\mu_{\theta}$. Then prices reveal the parameters of consumer preferences. If $\sigma_{\nu}=0$ and $\sigma_{\theta}>0$, $\pi_{2}=B$ and $\pi_{1}=\mu_{\nu}$. These two polar cases are discussed in Rosen (1974). Only in these two polar cases do prices directly reveal consumer preferences or firm productivities respectively. Similar results hold when $z, \theta$, and $\nu$ are vectors. We next turn to an analysis of identification in the general hedonic model. 


\section{Identification}

The most direct approach to estimating hedonic models is to solve the second order differential equation (3) implied by equilibrium for $P(z)$ in terms of the parameters of preferences, technology and the distributions of tastes and productivity and to jointly estimate the demand function corresponding to (2), the supply function corresponding to (1) and the distributions of unobservable preference and technology heterogeneity $\left(f_{\eta}\right.$ and $\left.f_{\varepsilon}\right)$ exploiting all of the information in the equilibrium conditions including data on demand, supply and the pricing function.

Rosen (1974) suggested an intuitively plausible and computationally simpler two step estimation procedure that has been widely criticized. In step 1 of his procedure, the analyst estimates $P(z)$ from market data. In step 2 , the analyst uses first order conditions in conjunction with the marginal prices obtained from step 1 to recover preferences and technology respectively. Suppose that consumer and firm first order conditions (1) and (2) are linear and $z$ is a scalar. These are exactly the first order conditions (4) and (5) of the Tinbergen model. (The scalar assumption is made only to simplify the argument and is not essential.) In this case, for the consumers, equation 
(1) would be of the form

$$
P_{z}(z)-A z+\theta_{0}+\theta_{1}^{\prime} x+\varepsilon=0
$$

For the firms, equation (2) would be of the form

$$
-B z+\nu_{0}+\nu_{1}^{\prime} y+\eta-P_{z}(z)=0
$$

Suppose further that the pricing function is quadratic as in Tinbergen (1956). Then the first stage of Rosen's procedure would be to estimate the pricing function $P(z)=\pi_{0}+\pi_{1} z+\frac{1}{2} \pi_{2} z^{2}$ and recover estimates of $\widehat{\pi}_{1}$ and $\widehat{\pi}_{2}$ ("^»" denotes estimate) and the marginal prices $\widehat{P}_{z}(z)=\widehat{\pi}_{1}+\widehat{\pi}_{2} z$. The second stage substitutes the estimated prices into (9) and (10) and estimates the curvature parameters: Thus, Rosen proposed to estimate $B$ and $\nu_{1}$ from the least squares regression

$$
\widehat{P}_{z}(z)=\hat{\pi}_{1}+\hat{\pi}_{2} z=\nu_{0}+\nu_{1}^{\prime} y-B z+\eta
$$


A parallel proposal for preferences estimates $A$ and $\theta_{1}$ from the regression

$$
\widehat{P}_{z}(z)=\hat{\pi}_{1}+\hat{\pi}_{2} z=-\theta_{0}-\theta_{1}^{\prime} x+A z-\varepsilon .
$$

James Brown and Harvey Rosen (1982) analyze this method. They interpret (11) and (12) as linearized approximations to the general first order conditions for the model. The linear-quadratic-normal model of Tinbergen (1956) is the framework in which these approximations are exact.

In this approximation interpretation, the distributions of $\eta$ and $\varepsilon$ are kept in the background. Standard linear econometric methods are applied to identify the parameters of (11) and (12) and connections among the parameters of preferences, technology and the distributions of tastes and productivity are not made explicit. Issues of identification are confused with issues of estimation. Common to an entire genre of empirical economics, this literature focuses on finding "good instruments" and misses basic sources of identification in hedonic models.

Starting from (11) and (12), Brown and Rosen (1982) make three points that have been reiterated in the subsequent empirical literature. Point One: Identification Can Only Be Obtained Through Arbitrary Functional Form 
Assumptions. Since $z$ is on both sides of (11) and (12), by a property of least squares, a regression using the constructed price $\widehat{P}_{z}(z)=\hat{\pi}_{1}+\hat{\pi}_{2} z$ as the dependent variable in (11) or(12) only identifies $\pi_{2}$. In general, $\pi_{2}$ does not identify any technology or preference parameter. In the special cases where there is no variation in preference parameters $\varepsilon$ or where there is no dispersion in $\eta, \pi_{2}$ identifies preference $(A)$ or production parameters $(B)$ respectively (See Rosen, 1974 or Ekeland, Heckman, and Nesheim, 2002).

However, if the constructed price is a nonlinear function of $z$, this argument no longer holds. The nonlinear variation in $\widehat{P}_{z}(z)$ gives an added piece of information that can help to identify technology and preference parameters. This identification strategy works because it rules out collinearity between $z$ and $\widehat{P}_{z}(z)$, but such nonlinearity is widely viewed as an artificial source of identification that is thought to be "arbitrary." Theorem 1 in Ekeland, Heckman, and Nesheim (2002) proves that this nonlinearity is a generic property of equilibrium in the hedonic model. In a parametric framework, Nesheim (2001) shows that nonlinearity is a robust feature of a hedonic economy with social interactions. In the context of the Tinbergen economy, this nonlinearity is generic.

Point Two: Endogeneity. Even if such "arbitrary" assumptions are made, 
so that one can use the nonlinearity in $\widehat{P}_{z}(z)$ to help identify the parameters and circumvent Point One, one still faces standard endogeneity problems. $z$ is correlated with $\eta$ and $\varepsilon$ in (11) and (12) respectively. Moreover, exclusion restrictions from the other side of the market cannot be justified. The equilibrium matching condition requires that $\eta=-\varepsilon-(A+B) z-\theta_{0}-\theta_{1}^{\prime} x+\nu_{0}+\nu_{1}^{\prime} y$ so that conditional on $z$ there is a functional and statistical dependence connecting $\varepsilon, \eta, z$ and the regressors. Conditional on $z, \eta, \varepsilon, x$ and $y$ become stochastically dependent even if in the underlying population initially they are mutually independent.

With data from a single market, one is forced to hunt for "clever" instruments with a questionable economic basis. Thus, even if "arbitrary" nonlinearities are invoked, standard instruments may be lacking. In Ekeland, Heckman, and Nesheim (2002) we show that the economics of the model guarantees valid instruments even though there are no exclusion restrictions. In the particular case of equation $(10)$, when $P_{z}(z)$ is nonlinear, $E(z \mid y)$ is not a linear function of $y$ and so can be used as an instrument for $z$ in this equation. Hence, generically, $E(z \mid y)$ is a valid instrument for $z$. This is discussed at more length below and holds more generally.

Point Three: Use of Multimarket Data. Rosen (1974), Brown and Rosen 
(1982), Epple (1987), and Kahn and Lang (1988) consider estimation of the first order conditions using multimarket data either across regions or across time in the same region. In this case, if we assume that preference parameters common across agents remain constant across markets while distributions of individual heterogeneity vary across markets, we can use cross market variation in prices and location choices to estimate the common preference parameters. However, this identification strategy relies on assumptions that can be tested if hedonic models can be identified in a single market. Using the techniques we discuss later in this paper, the structure of hedonic models can be estimated and identified using data from a single market for a class of additive parametric structures that includes the linear model as a special case.

Our results invalidate the interpretation that has been given to Brown and Rosen's criticism. What has been interpreted as an identification failure is in fact the failure of an estimation procedure, coupled with an approach that disregards basic sources of identification and mainly focuses on finding exclusion restrictions. In Ekeland, Heckman, and Nesheim (2002) and in Heckman, Matzkin, and Nesheim (2002) we show that when there are no unobservable attributes and some structure is put on preferences and tech- 
nologies, everything can be identified up to normalizations using single market data. In particular, Ekeland, Heckman, and Nesheim (2002) show that putting an additive structure on preferences is sufficient for identification. We describe these results in the next section. Alternatively, if the additive structure is too restrictive or is rejected by the data, Heckman, Matzkin, and Nesheim (2002) shows that in the nonadditive case alternative assumptions on preferences can lead to identification. We describe these results in section 4.2. Both sets of results consider the case where $z$ is unidimensional.

\subsection{Identification of the additive model}

To show that the preferences and technologies generating an hedonic equilibrium price function can be identified up to normalizations, using single market data and without any exclusion restrictions, suppose that $z$ is one dimen-

sional. Assume further that for unknown functions $M_{f}$ and $n_{f}$, the production function, $\Gamma(z, y, \eta)$, of a typical firm is $\Gamma(z, y, \eta)=M_{f}(z)+z n_{f}(y)+z \eta$. The firms' first order condition for profit maximization (2) becomes

$$
P_{z}(z)=m_{f}(z)+n_{f}(y)+\eta
$$


where $m_{f}(z)=\partial M_{f}(z) / \partial z$, and the second order condition is $\partial m_{f}(z) / \partial z-$ $P_{z z}(z)<0$. This is a special case of $(2)$ and is a significant generalization of (10) ; it reduces to (10) when $m_{f}$ and $n_{f}$ are linear functions.

In a parallel manner, we may assume that the marginal utility is also of an additive form. The first order condition (1) of the worker becomes

$$
P_{z}(z)=m_{w}(z)+n_{w}(x)+\varepsilon
$$

for some unknown functions $m_{w}$ and $n_{w}$. These equations are the empirical equations we seek to estimate and the equations that generate the equilibrium of the model through equation (3).

In the empirical analysis, we focus on the firms' equation. The analysis is analogous for the workers. We have a dataset with observations on $N$ firms drawn at random from a single market. For each firm, we observe the vector $\left(P_{z}(z), z, y\right)$. These data are generated from the equilibrium of a single hedonic market. This implies that for each firm, equation (13) holds where $\eta$ is unobservable to the econometrician, and $m_{f}(z), n_{f}(y)$, and $F_{\eta}$, the distribution of $\eta$, are to be estimated. We assume $P_{z}(z)$ is known to focus on the issue of identification and estimation of the structural parameters. In all 
cases if $P_{z}(z)$ is unknown because there is measurement error in prices, then a two stage procedure can be implemented a la Rosen (1974). First, estimate $P_{z}(z)$, then proceed to estimate (13) replacing the true price slope with the estimated slope. Alternatively, (13) and the equilibrium price equation (3) can be estimated simultaneously.

Much of the identification analysis is conducted independently of the equilibrium equation (3); it studies the conditional cumulate distribution function $(\mathrm{CDF})$ of $z$ implied by (13) and in particular makes use of the transformation function ${ }^{5}$

$$
T(z)=P_{z}(z)-m_{f}(z) .
$$

In terms of this function, the CDF of $z$ is

$$
F_{z \mid y}(z, y)=F_{\eta}\left(T(z)-n_{f}(y)\right)
$$

where $F_{z \mid y}(z, y)$ is the CDF of conditional on $y$ evaluated at the point $(z, y)$ and $F_{\eta}$ is the CDF of $\eta$. Differentiating (14) with respect to $z$ and $y_{i}$, the $i^{\prime}$ th

\footnotetext{
${ }^{5}$ See Horowitz (1996).
} 
component of the vector $y$, we have

$$
\begin{gathered}
\frac{\partial F_{z \mid y}(z, y)}{\partial z}=f_{\eta}\left(T(z)-n_{f}(y)\right) \frac{\partial T(z)}{\partial z} \\
\frac{\partial F_{z \mid y}(z, y)}{\partial y_{i}}=-f_{\eta}\left(T(z)-n_{f}(y)\right) \frac{\partial n_{f}(y)}{\partial y_{i}} .
\end{gathered}
$$

Taking the ratio of the derivatives we have

$$
\frac{-\frac{\partial F_{z \mid y}(z, y)}{\partial z}}{\frac{\partial F_{z \mid y}(z, y)}{\partial y_{i}}}=\frac{\frac{\partial T(z)}{\partial z}}{\frac{\partial n_{f}(y)}{\partial y_{i}}}
$$

Defining $h_{i}(z, y)=\ln \left(\frac{-\frac{\partial F_{z \mid y}(z, y)}{\partial z}}{\frac{\partial F_{z \mid y}(z, y)}{\partial y_{i}}}\right)$, this equation can be written

$$
h_{i}(z, y)=\ln \left(\frac{\partial T(z)}{\partial z}\right)-\ln \frac{\partial n_{f}(y)}{\partial y_{i}}
$$

This implies that $h_{i}(z, y)=h_{0}+h_{1}(z)+h_{2}(y)$ as shown in Ekeland, Heckman, and Nesheim (2002) and further that

$$
T(z)=R_{1}+K_{1} \int_{0}^{z} \exp \left(h_{1}(s)\right) d s
$$




$$
n_{f}(y)=C_{0}+K_{1} \exp \left(-h_{0}\right) \int_{0}^{y} \exp \left(-h_{2}(s)\right) d s
$$

for some $R_{1}, K_{1}$, and $C_{0}$. Since $h_{0}, h_{1}$, and $h_{2}$ can be estimated by estimating the CDF $\widehat{F}_{z \mid y}$ the functions $T(z)$ and $n_{f}$ can be estimated up to the three unknown constants. This in turn determines $m_{f}(z)=P_{z}-T(z)$. Fixing the constants and fixing $y$, the CDF of $\eta$ can then be estimated as

$$
\widehat{F}_{\eta}(e)=\widehat{F}_{z \mid y}(z(e), y)
$$

where $z(e)$ satisfies

$$
e=-R_{1}-K_{1} \int_{0}^{z(e)} \exp \left(h_{1}(s)\right) d s-C_{0}-K_{1} \exp \left(-h_{0}\right) \int_{0}^{y} \exp \left(-h_{2}(s)\right) d s
$$

Thus, the parameters $m_{f}(z), n_{f}(y)$, and $F_{\eta}(\eta)$ are identified up to the constants $R_{1}, K_{1}$, and $C_{0}$. The derivation of this results suggests an estimation procedure. First estimate $F_{z \mid y}$ and calculate $h_{i}(z, y)$. Then using $P_{z}(z)$ and the definition of $T(z)$, recover $m_{f}, n_{y}$, and $F_{\eta}$ from $(16),(17)$, and (18). We develop this further in the next section.

The above procedure leaves the 3 constants undetermined. Additionally, we can recover the parameter $K_{1}$ if more information is available. If for 
example, total output of the firm is observable, this information can be used to recover an estimate of $K_{1}$. This is developed further in Ekeland, Heckman, and Nesheim (2002). Alternatively, if it is known that $m_{f}(z)$ belongs to a known finite dimensional vector space $V$ so that $m_{f}(z)=\sum_{j=1}^{M} \theta_{j} \xi_{j}(z)$ where $\xi_{j}$ are the basis functions of the vector space, then we can go one step further and recover the unknown parameter $K_{1}$.

To see this,define $\widetilde{T}(z)=\int_{0}^{z} \exp \left(h_{1}(s)\right) d s$. Then using (16) and the definition of $T(z)$

$$
\widetilde{T}(z)=\frac{P_{z}(z)-\sum_{j=1}^{M} \theta_{j} \xi_{j}(z)-R_{1}}{K_{1}} .
$$

Theorem 2 in Ekeland, Heckman, and Nesheim (2002) proves that generically $P_{z}(z)$ does not belong to $V$. That is, generically $P_{z}(z)$ is linearly independent of $m_{f}(z)$. As a result, a regression of $\widetilde{T}(z)$ on $P_{z}(z)$, a constant, and the functions $\xi_{j}$ for $j=1, \ldots, M$ will recover $K_{1}$.

This procedure suggests a two step estimator for $K_{1}$. First estimate $\widetilde{T}(z)$ and then run the regression of $\widetilde{T}(z)$ on $P_{z}(z), \xi_{j}$, and a constant. $\widehat{K}_{1}$ is the inverse of the coefficient on $P_{z}(z)$. Our experience with this estimator to date is unfavorable. We develop an alternative estimator in section 5.1 that estimates a semiparametric version of the model using semiparametric 
maximum likelihood where the Monte Carlo results are much better.

\subsection{Identification of the nonadditive model}

Heckman, Matzkin, and Nesheim (2002) have shown that to be able to identify the preferences and technologies generating an hedonic equilibrium price function, it is not necessary that the marginal utility and marginal product functions be additive functions of the types specified in Section 4.1. Under certain conditions, one can identify nonadditive marginal utilities and nonadditive marginal product functions, using single market data and no exclusion restrictions. Nonadditive specifications for either of these marginal functions are capable of generating a much richer set of equilibria, such as, for example, equilibria exhibiting bunching. Hence, it is important to be able to allow for these more flexible types of specifications when estimating preferences and technologies.

Since the arguments for the identification and estimation of the marginal product function are analogous to those used to establish the identification of the marginal utility function, we will discuss only the latter. From the analysis in Section 2, it follows that from the first and second order conditions of profit maximization by a worker, we can establish the existence of 
a supply function $z=s(x, \varepsilon)$, where $z$ denotes the quality or type of labor supplied by a worker with observable characteristic $x$ and unobservable characteristic $\varepsilon$. The function $s$ is strictly increasing in $\varepsilon$ if $U_{z \varepsilon}<0$. Assume, as in the previous sections, that $\varepsilon$ is distributed independently of $x$, then by the arguments introduced in Matzkin (1999), and further developed in Matzkin (2003), it follows that, subject to some normalizations, the function $s$ and the distribution of $\varepsilon$ can be nonparametrically identified from the conditional distribution of $z$ given $x$. Knowledge of the function $s$ and of the distribution of $\varepsilon$, together with knowledge of $P_{z}$, allow one to identify the marginal utility function, from the first order conditions of utility maximization. This last step requires a separability restriction on the marginal utility function, of the type studied in Matzkin (2002, 2003).

To present one such set of separability restrictions and normalizations, suppose that for some unknown function $m$, which is strictly increasing in its first argument and strictly decreasing in its second argument

$$
U_{z}(z, x, \varepsilon)=m(q(z, x), \varepsilon)
$$

where $q$ is a known function, which is strictly increasing in each argument. 
Normalize the values of the unknown function $m$, by requiring that for some value $\bar{x}$ of $x$, and for all $t$

$$
m(q(t, \bar{x}), t)=P_{z}(t)
$$

Then, as it is shown in Heckman, Matzkin, and Nesheim (2002), under these restrictions and normalizations, both the distribution of $\varepsilon$ and the function $m$, are nonparametrically identified from the conditional distribution of $z$ given $x$.

The weak separability restriction in $U_{z}$ allows one to recover the marginal utility when the supply function is given. The normalization that fixes the value of the function $m$ at one point of $x$ allows one to recover the supply function $s$ and the distribution of $\varepsilon$ from the conditional distribution of $z$ given $x$. To see this last point, note that the normalization restriction together with the first order conditions imply that for all $\varepsilon$

$$
s(\bar{x}, \varepsilon)=\varepsilon
$$


since, when $x=\bar{x}$ and $z=\varepsilon$

$$
U_{z}(\varepsilon, \bar{x}, \varepsilon)=m(q(\varepsilon, \bar{x}), \varepsilon)=P_{z}(\varepsilon) .
$$

The strict monotonicity of $s$ in $\varepsilon$ and the statistical independence between $x$ and $\varepsilon$, imply that, for all values of $x$ and $\varepsilon$,

$$
F_{\varepsilon}(e)=\operatorname{Pr}(\varepsilon \leq e)=\operatorname{Pr}(\varepsilon \leq e \mid x)=\operatorname{Pr}(s(x, \varepsilon) \leq s(x, e))
$$

and that

$$
\operatorname{Pr}(s(x, \varepsilon) \leq s(x, e))=\operatorname{Pr}(z \leq s(x, e) \mid x)=F_{z \mid x}(s(x, e)) .
$$

Letting $x=\bar{x}$, this implies that

$$
F_{\varepsilon}(e)=F_{z \mid \bar{x}}(s(\bar{x}, e))=F_{z \mid \bar{x}}(e)
$$

Hence, we can recover the distribution of $\varepsilon$ from the conditional distribution of $z$ given $x=\bar{x}$. Next, since for all $x$ and $e, F_{\varepsilon}(e)=F_{z \mid x}(s(x, e))$, it follows 
that, under conditions guaranteeing that $F_{z \mid x}$ is strictly increasing,

$$
s(x, e)=F_{z \mid x}^{-1}\left(F_{\varepsilon}(e)\right)=F_{z \mid x}^{-1}\left(F_{z \mid \bar{x}}(e)\right)
$$

Hence, we can recover the function $s$ from the conditional distribution of $z$ given $x$. (See Matzkin (2003) for details.)

To see that under the separability restriction, the function $m$ is identified, let $\left(t_{1}, t_{2}\right)$ denote a vector on the domain of $m$. Find $x^{*}$ such that

$$
q\left(s\left(x^{*}, t_{2}\right), x^{*}\right)=t_{1}
$$

Then, since by the definition of $m$ and the first order conditions of utility maximization

$$
m\left(t_{1}, t_{2}\right)=U_{z}\left(s\left(x^{*}, t_{2}\right), x^{*}, t_{2}\right)=P_{z}\left(s\left(x^{*}, t_{2}\right)\right)
$$

it follows that, from knowledge of the function $s$ and of the marginal price function, we can recover the function $m$, which gives the values of the marginal utility function (See Heckman, Matzkin, and Nesheim (2002) for details). Estimation of the function $m$ and the distribution of $\varepsilon$ follows the steps 
described above and is detailed in section 5.2.

\section{Estimation}

Several estimation techniques are available to implement the analysis presented in sections 4.1 and 4.2. All make use of the structure that the additive and nonadditive models impose on $F_{z \mid y}$. In the case of the nonadditive model, a fully nonparametric estimator is described in section 5.2. In the case of the additive model, this information is sufficient to identify the structural parameters up to location and scale. Additionally, if parametric restrictions are placed on $m_{f}(z)$ in the additive model, the generic nonlinearity of the hedonic model can be exploited to estimate the scale. To show how this scale parameter can be estimated and for ease of exposition in developing an estimator and presenting the estimation results for the additive model, we focus on a semiparametric estimator that exploits knowledge of the functional forms of $m_{f}(z)$ and $n_{f}(y)$ but which makes no further assumptions on the distribution of $\eta$. This estimator is semiparametric in that $m_{f}$ and $n_{f}$ are known up to a finite dimensional parameter set while the distribution of $\eta$ is unknown. We also restrict the exposition to the case where the dimension of 
$y$ is 1 . We develop this estimator for the additive model in the next section.

\subsection{Estimation of the additive model}

In our limited Monte Carlo investigations, we generate data from and develop an estimator for specifications of $m_{f}(z)$ and $n_{f}(y)$ in (13) that are linear in the parameters. The first order condition (1) may then be written:

$$
P_{z}(z)=\sum_{i=0}^{N_{z f}} \beta_{i} z^{i}+\sum_{j=0}^{N_{\nu}} \nu_{j} y^{j}+\eta
$$

We assume that a random sample of data on $\left(P_{z}\left(z_{n}\right), P_{z z}\left(z_{n}\right), z_{n}, y_{n}\right)$ for $n=1, \ldots, N$ are available for a single market. To focus on the estimation of preferences or technology we assume that $P_{z}(z)$ and $P_{z z}(z)$ are known. If instead prices were observed measured with error, the technique described below would need to be augmented to allow for estimation of the pricing function. The technique exploits all the information in the model and uses the generic nonlinearity in the model to identify not only the shape of the marginal product function but also the scale.

$$
\text { Using } m_{f}(z)=\sum_{i=0}^{N_{z f}} \beta_{i} z^{i} \text { and } n_{f}(y)=\sum_{j=0}^{N_{\nu}} \nu_{j} y^{j} \text {, the density of the } n^{\prime} t h
$$


observation $z_{n}$ conditional on $y_{n}$ is

$f_{z \mid y}\left(z_{n}, y_{n}\right)=\widehat{f_{\eta}}\left(P_{z}\left(z_{n}\right)-\sum_{i=0}^{N_{z f}} \beta_{i} z_{n}^{i}-\sum_{j=0}^{N_{\nu}} \nu_{j} y_{n}^{j}\right) \cdot\left(P_{z z}\left(z_{n}\right)-\sum_{i=1}^{N_{z f}} i \beta_{i} z_{n}^{i-1}\right)$

where

$$
\widehat{f}_{\eta}(\eta)=(N h)^{-1} \sum_{k=1}^{N} K\left(\frac{\eta_{k}-\eta}{h}\right)
$$

is the kernel density estimator of $f_{\eta}$. We propose to estimate the parameters by maximizing the likelihood function for the sample.

Let $\beta$ and $\nu$ be the vectors of parameters excluding $\beta_{0}$ and $\nu_{0}$. The log-likelihood function for the sample is

$$
\begin{aligned}
l\left(\beta, \nu, \beta_{0}, \nu_{0}\right)= & \sum_{n=1}^{N} \ln \widehat{f}_{\eta}\left(P_{z}\left(z_{n}\right)-\beta_{0}-\sum_{i=1}^{N_{z f}} \beta_{i} z_{n}^{i}-\nu_{0}-\sum_{j=1}^{N_{\nu}} \nu_{j} y_{n}^{j}\right)+ \\
& \sum_{n=1}^{N} \ln \left(P_{z z}\left(z_{n}\right)-\sum_{i=1}^{N_{z f}} i \beta_{i} z_{n}^{i-1}\right)
\end{aligned}
$$

which simplifies to

$$
\begin{aligned}
& l(\beta, \nu)=\sum_{n=1}^{N} \ln \left((N h)^{-1} \sum_{k=1}^{N} K\left(\xi_{k n}\right)\right)+ \\
& \sum_{n=1}^{N} \ln \left(P_{z z}\left(z_{n}\right)-\sum_{i=1}^{N_{z f}} i \beta_{i} z_{n}^{i-1}\right)
\end{aligned}
$$


where

$$
\xi_{k n}=\frac{P_{z}\left(z_{k}\right)-P_{z}\left(z_{n}\right)-\sum_{i=1}^{N_{z f}} \beta_{i}\left(z_{k}^{i}-z_{n}^{i}\right)-\sum_{j=1}^{N_{\nu}} \nu_{j}\left(y_{k}^{j}-y_{n}^{j}\right)}{h}
$$

It is immediately obvious that $\beta_{0}$ and $\nu_{0}$ are not identified; they drop out of the expression for $\xi_{k n}$. We ignore them in the rest of the discussion. It is also immediate that the parameters $\beta$ are identified if and only if $P_{z}(z)$ is not a polynomial of degree less than or equal to $N_{z f}$. Theorem (1) in Ekeland, Heckman, and Nesheim (2002) guarantees that generically the slope parameters in $\beta$ are identified. The maximum likelihood estimators of $\beta$ and $\nu$ are found by maximizing the log-likelihood subject to $P_{z z}\left(z_{n}\right)-\sum_{i=1}^{N_{z f}} i \beta_{i} z_{n}^{i-1}>$ 0 for all $n$. An estimator of $f_{\eta}$ is

$$
\widehat{f}_{\eta}(\eta)=(N h)^{-1} \sum_{k=1}^{N} K\left(\frac{\widehat{\eta}_{k}-\eta}{h}\right)
$$

where $\widehat{\eta}_{k}=P_{z}\left(z_{k}\right)-\sum_{i=1}^{N_{z f}} \widehat{\beta}_{i} z_{k}^{i}-\sum_{j=1}^{N_{\nu}} \widehat{\nu}_{j} y_{k}^{j}$. 


\subsection{Estimation of the nonadditive model}

The most direct procedure to estimate the function $m$ and of the distribution of $\varepsilon$ in the nonadditive model follows the steps described in section 4.2. First, $F_{z \mid x}$ is estimated nonparametrically using data on the joint distribution of $(z, x)$. this nonparametric estimator is $\widehat{F}_{z \mid x}$ Then this estimator is substituted into equation (20). This defines an estimator of $s(x, \varepsilon)$, $\widehat{s}(x, e)=\widehat{F}_{z \mid x}^{-1}\left(\widehat{F}_{z \mid \bar{x}}(\varepsilon)\right)$. Heckman, Matzkin, and Nesheim (2002) have shown that when $\widehat{F}_{z \mid x}$ is a kernel estimator for $F_{z \mid x}$, defined by

$$
\widehat{F}_{z \mid x}(t)=\frac{\sum_{i=1}^{N} \widetilde{K}\left(\frac{t-z_{i}}{\sigma}\right) K\left(\frac{x-x_{i}}{\sigma}\right)}{\sum_{i=1}^{N} K\left(\frac{x-x_{i}}{\sigma}\right)}
$$

where $K$ is a kernel function, $\widetilde{K}$ is the integral of a kernel function, and $\sigma$ is a bandwidth, the estimators for the distribution of $\varepsilon$ and for the function $m$ are consistent and asymptotically normally distributed. Finally, using this estimator $\widehat{s}(x, \varepsilon)$, data on the marginal price $P_{z}(z)$, and equations (21) and (22), $m$ is estimated as

$$
m\left(t_{1}, t_{2}\right)=P_{z}\left(\widehat{s}\left(x^{*}, t_{2}\right)\right)
$$


where $x^{*}$ solves $q\left(\widehat{s}\left(x^{*}, t_{2}\right), x^{*}\right)=t_{1}$.

\section{Simulation and estimation results}

In this section, we present simulation results from a range of specifications of 3 basic hedonic models. In each case, these simulations show the shape of the equilibrium pricing function, the population density at each point $z$, and the generic nonlinearity of the hedonic model. The accompanying estimation results demonstrate the performance of the estimation techniques described in sections 5.1 and 5.2 .

Models 1 and 2 are examples of additive hedonic models. For these models we study the equilibria for several sets of parameter values and study how the shape of the equilibrium price varies with alternate parameter values. Then we simulate data from these sample hedonic economies and test the performance of the estimation techniques on the simulated data. For each model we simulate data from 15 parameter specifications. For each specification, we generate 100 independent samples each with 1000 observations. Using these simulated data, we estimate the parameters using the technique described in section 5.1. The mean and the variance of the parameter es- 
timates are compared with the true parameter values used to generate the simulated data.

Model 3 is an example of a nonadditive hedonic model. The estimation technique that is used to estimate the parameters of the additive models is not applicable to data generated from the nonadditive economy. Instead, the technique from section 5.2 must be used. For model 3, we simulate data from a sample economy and study the performance of the nonadditive model estimator described in section 5.2.

All models are completely specified by the firms' technology $\Gamma(z, y, \eta)$, the workers' utility $U(z, x, \varepsilon)$, and the distributions of firm and worker heterogeneity $f_{y}, f_{\eta}, f_{x}$, and $f_{\varepsilon}$. For each model, these objects are specified and then standard numerical methods are used to approximate $P_{z}(z)$ the solution to the equilibrium differential equation (3). Throughout the exposition below $\phi(x, \mu, \Sigma)$ denotes the density function of a normal random variable with mean $\mu$ and covariance $\Sigma$. Model 1 is a quadratic model with nonnormal heterogeneity. Model 2 is an additive model in which $m_{f}, n_{f}, m_{w}$, and $n_{w}$ are low degree polynomials. For both models we discuss features of the equilibrium price function, simulate data, and estimate the structural parameters describing firm technologies. Model 3 is a nonadditive model in 
which firms are homogenous and workers have Cobb-Douglas utility. It is described further below.

\subsection{Model 1: Specification and simulation results}

The simplest generalization of the normal-quadratic Tinbergen model is the quadratic hedonic model with non-normal heterogeneity. This model speci-

fication imposes $N_{z f}=1$ and $N_{\nu}=1$ in equation (23) and in the analogous equation for workers. However, in contrast to the classical Tinbergen model, this model allows the heterogeneity parameters to be distributed as a mixture of normals. Details of model 1 are given in Table A1 in appendix A. This table details the exact functional forms that describe the model.

For this model, preliminary investigations simulated pricing functions for a large number of specifications. In all of these specifications, parameters were restricted to cases where worker and firm heterogeneity were distributed as mixtures of normals each with two components in the mixture. The extreme cases of these specifications include the Tinbergen-normal case when the weights on the two components of the mixture are 0 and 1 or 1 and 0 . In this set of specifications the parameters that most affected the shape of the pricing function were the mean and variance of worker and firm heterogeneity 
and the weights on the components of the mixture of normals distribution. In all cases the curvature of the pricing function is a linear combination of the curvatures of worker preferences and firm technologies. (See Ekeland, Heckman, and Nesheim, 2002 and Heckman, Matzkin, and Nesheim, 2002).

From among the specifications investigated we selected 15 to report here. The specifications are detailed in Tables A2 and A3. The tables list 5 specifications. For each of these specifications we allowed the parameter $\lambda_{\eta 1}=\lambda_{\varepsilon 1}=\lambda$ to vary from 0.1 , to 0.5 , to $0.9 .{ }^{6}$ Thus there are 3 variations of each of 5 specifications. Specifications 1 and 5 represent two extreme cases and specifications 2-4 represent linear combinations of those cases.

The price functions associated with each specification are depicted in Figures 1-20. For each economy, we display the slope of the price function, the population density at each location $z$, and the curvature of the price function. Figures 1-10 show how the slope, the curvature, and the density vary as $\lambda$ varies from 0.5 to 0.9 to 1.0 . Figures $11-20$ show how the slope, curvature, and the density vary as $\lambda$ varies from 0.0 to 0.1 to 0.5 . The figures largely tell the same story. When $\lambda=0.0$ or 1.0 , the slope is a straight line, the curvature is constant, and the density is a normal density. However,

\footnotetext{
${ }^{6}$ The cases in which $\lambda=0$ and $\lambda=1$, the Tinbergen, normal-quadratic cases, are displayed in the figures for comparison.
} 
when $\lambda=0.1$ or 0.9 the slope is not a straight line, the curvature is not constant, and the density is not a normal density. These deviations from the Tinbergen case are even stronger when $\lambda=0.5$. The figures also show that the slope of the price function deviates most sharply from a straight line in the two extreme cases, specification 1 and specification 5. All the figures show however, that the curvature deviates strongly from a constant when heterogeneity is not normal. The closer the distribution of heterogeneity is to normal, the closer the curvature is to a constant.

\subsection{Model 1: Estimation results}

The pictures described in the previous section show that there is nonlinearity in the marginal price function and non-constancy in the curvature of the price function when heterogeneity is not normally distributed in the population in this quadratic model. The question remains, is that nonlinearity sufficient to estimate the structural parameters in the model with precision? To shed light on this question, we generated data from the 15 specifications described in the previous section and in Tables A2 and A3 and estimated the Model 1 
version of equation (23),

$$
P_{z}(z)=-B z+\nu_{0}+\nu_{1}^{\prime} y+\eta
$$

We generated 100 datasets each of sample size 1000 for each specification and then estimated the parameters $B$ and $\nu_{1}$ using the technique discussed in section 5.1. The results are displayed in Tables A4, A5, and A6. Table A4 displays results for each of the 5 specifications listed when $\lambda_{\eta 1}=\lambda_{\varepsilon 1}=$ $\lambda=0.5$. Table A5 displays results for each specification when $\lambda=0.9$ and Table A6 displays results for each specification when $\lambda=0.1$. The contrast between Table A4 and Tables A5 and A6 give some indication of how much the precision of the results deteriorate when the economy is closer to the normal-quadratic Tinbergen economy which is not identified.

First consider the results in Table A4. The bias of the maximum likelihood (ML) estimator is never larger than 0.04. The standard errors range from 0.0349 in specification 1 to 0.287 in specification 4 . Specifications 3 and 4 have the highest standard errors. Looking at Tables A2 and A3, these are the specifications in which the two components of the mixtures of normals distribution are most similar. That is, these are the two specifications that 
are closest to being not identified.

Table A5 and A6 investigate how the results in Table A4 change when $\lambda=$ 0.9 and 0.1 . In these cases, the distributions or worker and firm heterogeneity are closer to being normally distributed and the price function is closer to being linear. In these cases, the ML results are essentially unchanged. The bias of the ML estimator is the same order of magnitude. The biggest increase is for specification 4 when $\lambda$ decreases from 0.5 to 0.1 . In this case, the bias of the ML estimator increases from 0.02 to 0.12 . This is still less than $7 \%$ of the parameter value. The standard errors of the estimates increase slightly. The biggest increase when $\lambda$ increases from 0.5 to 0.9 is in specification 1 when the standard errors increase from 0.0349 and 0.0518 to 0.12 and 0.194 . This is nearly a four-fold increase. Yet the standard errors after the increase are still only $12 \%$ and $20 \%$ of the parameter values.

The results indicate that the ML estimator can provide very good parameter estimates in a range of specifications. These results apply to the linear-quadratic model of Tinbergen with non-normal heterogeneity and to the linear approximations of Brown and Rosen (1982). Far from not being identified, the parameters are estimated with a high degree of precision. 


\subsection{Model 2: Simulation results}

The results in Ekeland, Heckman, and Nesheim (2002) apply to additive models more general than the quadratic model. Model 2 generalizes Model 1 by replacing the linear-quadratic terms in the production and utility functions with nonlinear terms. Model 2 is detailed in Table A7. The specification was chosen so that every function is a polynomial, $\nu(y)$ is strictly increasing $\left(\nu_{1}>0\right), \theta(x)$ is strictly increasing $\left(\theta_{1}>0\right)$, and $m_{f}(z)<m_{w}(z)$ This last restriction rules out bunching. The specification allows the curvatures of utility and preferences to be flexible and vary with $z$. It also allows for a flexible relation between preference heterogeneity $\nu(y)$ and observable firm traits $y$. While the power series representations detailed in Table A7 could be replaced with orthogonal polynomial representations or representations based on other basis functions, the power series representations were chosen for ease of exposition.

It was more costly in terms of computer time to simulate equilibria and generate data from model 2. We studied a more limited set of specifications. In particular 5 specifications were chosen at random from a compact parameter space. The five chosen are detailed in Tables A8 and A9. The parameters that were allowed to vary across specifications include $\beta, \nu_{1}, \mu_{\eta}, \sigma_{\eta}, \alpha_{0}, \alpha_{1}$, 
$\mu_{\varepsilon}$, and $\sigma_{\varepsilon}$. These parameters govern the curvature of the firms' technology, the minimum slope of $\nu(y)$, the mean and variance of unobservable firm heterogeneity, the curvature of worker preferences, and the mean and variance of worker heterogeneity.

Figures 21-30 display the slope of the price function, the curvature of the price function, and the density of $z$ for specifications 1 through 5. For each specification, three variations are plotted; one with $\lambda=0.5$, one with $\lambda=0.9$, and one with $\lambda=1.0$. Similar graphs depicting the case in which $\lambda=0.0$ and 0.1 are available from the authors upon request. Clearly a wide variety of shapes of the price function are possible. In all cases, it appears as if the price function might be well approximated by a quadratic or a cubic but there are sharp deviations from these shapes. Simple quadratic or cubic approximations to the price function would miss these important deviations. Also, noteworthy is the shape of the equilibrium density of $z$. In all the cases displayed, this density has many modes and is far from being a normal density. In particular, this model is capable of generating equilibria in which there are nearly gaps in the range of products marketed. In figure 21, the fraction of firms demanding $z<1$ is positive and large, the fraction demanding $z \in(1,2)$ is nearly zero (though positive), and the 
fraction demanding $z>2$ is positive and large. This gap in the product range reflects two factors, the distribution of heterogeneity in the population, and the curvature of preferences and technology.

The value of $\lambda$ does not appear to have large impacts on the slope, the price, or the density. It does cause deviations in the shapes of these objects but not large ones. This lack of impact is likely to be an artifact of the set of specifications investigated. In all specifications, the distribution of firm heterogeneity is determined by both the distribution of $\nu(y)$ and the distribution of $\eta$. Because $\nu(y)$ is a 5th order polynomial in $y$, the variance of $\nu(y)$ dominates the variance of $\eta$ in all specifications. Hence, $\eta$ only has small local effects on the equilibrium and does not have large non-local impacts. Nevertheless, the model is far from the normal model since $\nu(y)$ is far from being normal.

\subsection{Model 2: Estimation results}

Tables A10-A19 present the estimation results for model 2. Tables A10-A14 present results for specifications 1 through 5 when $\lambda=0.5$. Tables A15-A19 present results for the same specifications when $\lambda=0.9$. Results for the cases where $\lambda=0.1$ are omitted to economize on space. They are available from 
the authors upon request; they are largely similar to those presented.

In all the specifications estimated the ML estimator again performs very well. In Table A10 for instance the true value of $\beta_{1}$ is 0.623 . The average estimate is 0.637 . The bias is $2 \%$. This degree of bias is typical of all parameters estimates across all specifications. In all cases the bias has an order of magnitude never larger than 0.03 ; in the range of $2-3 \%$ of the parameter values. The standard error of the estimates of $\beta_{1}$ in Table A10 is 0.243 or $39 \%$ of the parameter value. In percentage terms this standard error is the fourth largest standard error for any parameter in any of the specifications. The other large standard errors are $\beta_{1}$ in Table A15, and $\beta_{1}$ in Tables A13 and A18. The largest standard error obtained is the standard error of the estimate of $\beta_{1}$ in Table A18 which is 0.405 or $63 \%$ of the true parameter value. Standard errors for all other parameter estimates in all specifications are much lower than this. In Table A10 the second largest standard error in percentage terms is the standard error on $\nu_{c 1}$ which is 0.256 or $13.8 \%$ of the parameter value. Most of the standard errors in Tables A10 through A19 range between $5 \%$ and $20 \%$.

Thus, the ML estimator performs very well. Despite the high degree of nonlinearity in these additive models, the technique recovers parameter 
estimates with small bias and reasonable standard errors.

\subsection{Model 3: Simulation and estimation results}

When the data reject the additive specifications above, alternative techniques are required. Some techniques for estimating nonadditive models are developed in sections 4.2 and 5.2. To evaluate the small sample properties of these estimators for economies where either the marginal utility function or the marginal product function, or both, are nonadditive in the unobservable characteristics, we consider an economy where workers differ in the value of the observable characteristic $x$ and the unobservable characteristic $\varepsilon$. To focus analysis on estimation of the utility functions we generate observations from an economy with homogenous production technologies. The marginal price function is given by the marginal product function. The specification that we use is described in Table 1.

Table 1: Model 3 Functional Forms

\begin{tabular}{|c|c|c|c|}
\hline Firm & Technology & $\Gamma(z)$ & $A z^{\alpha}$ \\
\hline Worker & Utility & $U(z, x, \varepsilon)$ & $B z^{\beta} x^{\beta-1} \varepsilon^{\delta}$ \\
\hline & Density of $x$ & $f_{x}(x)$ & $U[0.5,1.5]$ \\
\hline & Density of $\varepsilon$ & $f_{\varepsilon}(\varepsilon)$ & $U[3.0,4.0]$ \\
\hline
\end{tabular}


In this economy, profit maximization by each of the homogenous firms implies that the first order condition:

$$
A \alpha z^{\alpha-1}-P_{z}(z)=0
$$

and the second order condition:

$$
A \alpha(\alpha-1) z^{\alpha-2}-P_{z z}(z) \leq 0
$$

are satisfied. Since all the firms have the same production technology, the only possible equilibrium price function for this economy is given by

$$
P_{z}(z)=A \alpha z^{\alpha-1} .
$$

This is an example of Rosen's 1974 argument that when one side of the market is homogenous, the price function directly reveals parameters of that side of the market.

The first order condition for utility maximization of a worker with characteristics $(x, \varepsilon)$ is

$$
P_{z}(z)-B \beta z^{\beta-1} x^{\beta-1} \varepsilon^{\delta}=0
$$


and the second order condition is

$$
P_{z z}(z)-B \beta(\beta-1) z^{\beta-2} x^{\beta-1} \varepsilon^{\delta}<0
$$

Using the equilibrium price function, we get that the supply function of the worker, describing the quality of labor supplied is

$$
z=\left(\frac{A \alpha}{B \beta} x^{1-\beta} \varepsilon^{-\delta}\right)^{\frac{1}{\beta-\alpha}}
$$

as long as

$$
A \alpha(\alpha-1) z^{\alpha-2}-B \beta(\beta-1) z^{\beta-2} x^{\beta-1} \varepsilon^{\delta}<0
$$

The latter inequality is satisfied as long as

$$
A \alpha(\alpha-1)\left(\frac{A \alpha}{B \beta} x^{1-\beta} \varepsilon^{-\delta}\right)^{\frac{\alpha-2}{\beta-\alpha}}-B \beta(\beta-1)\left(\frac{A \alpha}{B \beta} x^{1-\beta} \varepsilon^{-\delta}\right)^{\frac{\beta-2}{\beta-\alpha}} x^{\beta-1} \varepsilon^{\delta}<0
$$

or

$$
(A \alpha)^{\frac{\beta-2}{\beta-\alpha}}(B \beta)^{\frac{\alpha-2}{\beta-\alpha}}\left(x^{1-\beta} \varepsilon^{-\delta}\right)^{\frac{\alpha-2}{\beta-\alpha}}[\alpha-\beta]<0
$$

Hence, when $A>0, B>0, \varepsilon>0$ and $x>0$, if $0<\alpha<\beta$, the equilibrium 
price function is

$$
P_{z}(z)=A \alpha z^{\alpha-1}
$$

and the supply function of a worker with characteristic $(x, \varepsilon)$ is

$$
z=\left(\frac{A \alpha}{B \beta}\right)^{\frac{1}{\beta-\alpha}} x^{\frac{1-\beta}{\beta-\alpha}} \varepsilon^{\frac{-\delta}{\beta-\alpha}}
$$

To evaluate the estimators for the marginal utility of the workers obtained using the estimators developed in Heckman, Matzkin, and Nesheim (2002), we will simulate observations for pairs $(x, z)$ generated by this supply function and the specifications described above for the distributions of $x$ and $\varepsilon$. We will require that for some strictly increasing function $m$

$$
U_{z}(z, x, \varepsilon)=m(z x, \varepsilon) .
$$

The utility function described in Table 1 satisfies this restriction. The true function $m$ is $m(z x, \varepsilon)=\beta B(z x)^{\beta-1} \varepsilon^{\delta}$. Assuming the true function is unknown and assuming the true distribution of $\varepsilon$ is unknown, we impose the 
normalization that, for all $t$ within the relevant domain,

$$
m(t \bar{x}, t)=P_{z}(t)
$$

Since the equilibrium marginal price function satisfies: $P_{z}(z)=A \alpha z^{\alpha-1}$, the normalization implies that

$$
m(t \bar{x}, t)=A \alpha t^{\alpha-1}
$$

We could choose any value for $\bar{x}$, and use a transformation to modify the true marginal utility function $m$ and the true distribution of $\varepsilon$ to be within the set of marginal utilities and distributions that are consistent with the normalization imposed by our particular choice of $\bar{x}$. However, for simplicity, we choose the value of $\bar{x}$ and of the other parameters to be such that the true function $m$ and the distribution of $\varepsilon$ are consistent with the normalization generated by that $\bar{x}$. Our choices for the parameters are

\begin{tabular}{|c|c|c|c|c|c|}
\hline$A$ & $\alpha$ & $B$ & $\beta$ & $\delta$ & $\bar{x}$ \\
\hline .55 & .3 & .15 & 1.1 & -.8 & 1. \\
\hline
\end{tabular}

The equilibrium marginal price function for this economy is depicted with a solid line in Figure 31. The dotted lines represent bounds on feasible 
marginal prices produced by the restriction that an equilibrium marginal price must satisfy equation (25) for some $(x, \varepsilon)$ and $(x, \varepsilon)$ are elements of a compact set.

Using these parameters and the derivations above we generated 100 independent samples each with 100 observations of $(z, x)$ pairs. Using these samples we estimated the distribution of $\varepsilon$, the workers' supply function $z=s(x, \varepsilon)$, and the marginal utility function $m$. To estimate the conditional distribution of $z$ given $x$, bandwidths were chosen by cross validation.

Figure 32 displays estimates of the distribution function of $\varepsilon$. In both panels of the figure, the solid line displays the true distribution function. The dashed line displays an estimate. The dashed lines in the lower panel display the median and average of the 100 estimates of the distribution function. These track the true distribution function quite closely. The maximum gap in the tails of the distribution is about 0.05 . The maximum gap outside the tails is negligible. The dotted lines in the panel plot the 5th and 95th percentile of the estimates. The maximum gap between these two quantiles and the true distribution function is about 0.2 . This graph shows that the nonparametric estimate of the distribution function tracks the true distribution function very well. 
Figures 33 and 34 plot the estimates of the supply function (26). The upper panels display 3-dimensional graphs of this function. The lower panels display cross sections of the function at particular values of $x$ and $\varepsilon$. Figure 33 illustrates how well a single estimate of the supply function can do. Figure 34 illustrates the median and average estimate of the 100 independent estimates. The dashed lines in the lower panels show that when $\varepsilon=3.45$ or when $x=0.62$, the median and average estimates track the true supply function with negligible error. The dotted lines depict the 5th and 95th percentile of these estimates.

Finally, Figures 35 and 36 portray estimates of the marginal utility function. Figure 35 show how well a single estimate can do, showing the three dimensional $m(z x, \varepsilon)$ in the upper panels and the marginal utility $m(z x, \varepsilon)$ for fixed values of $\varepsilon$ and $z x$ respectively in the lower two panels. Figure 36 illustrates the average and median estimates as well as the 5th and 95th percentile. When $\varepsilon$ is fixed at $\varepsilon=3.45$, the median and average estimates of $m(z x, 3.45)$, plotted with dashed lines, track the true function with negligible error. Similarly when $z x$ is fixed at $z x=3.56$, the median and average estimates are indistinguishable from the true function. Again, the dotted lines show the 5th and 95th percentile estimates. 


\section{Conclusion}

Much of previous analysis of hedonic models has neglected to consider the strong implications imposed by equilibrium on the data generated from a hedonic model. In particular, strong restrictions on preferences and technologies like additive separability impose a great deal of structure on the joint distribution of observable random variables. In this paper, we have developed these points in our discussion and illustrated them with two computational examples. The graphical displays of equilibrium prices in the array of models we consider demonstrate the strong nonlinearities that are generic features of hedonic models. Nonlinearity in these models is not arbitrary, but emerges quite naturally. This nonlinearity in conjunction with restrictions like additivity allow for identification and estimation of the hedonic model. In finite samples of size 1000, the semiparametric maximum likelihood technique performs well in recovering estimates of structural parameters in both the linear-quadratic model and in model 2 where the nonlinearities in the structure of the economy are more severe. Finally, in model 3, with sample sizes of only 100, the nonparametric techniques developed for nonadditive models work very well.

The structural parameter estimates obtained in these exercises are cru- 
cial for any general equilibrium analysis that seeks to address the welfare consequences of policy changes in hedonic markets. They are also crucial for employing hedonic methods to correct cost of living indices for changes in the characteristics of marketed goods. Without estimation of the structural parameters underlying a hedonic market, changes in the hedonic pricing relationship are uninterpretable. 
Figure 1: Model 1, Specification 1: Slope of price function
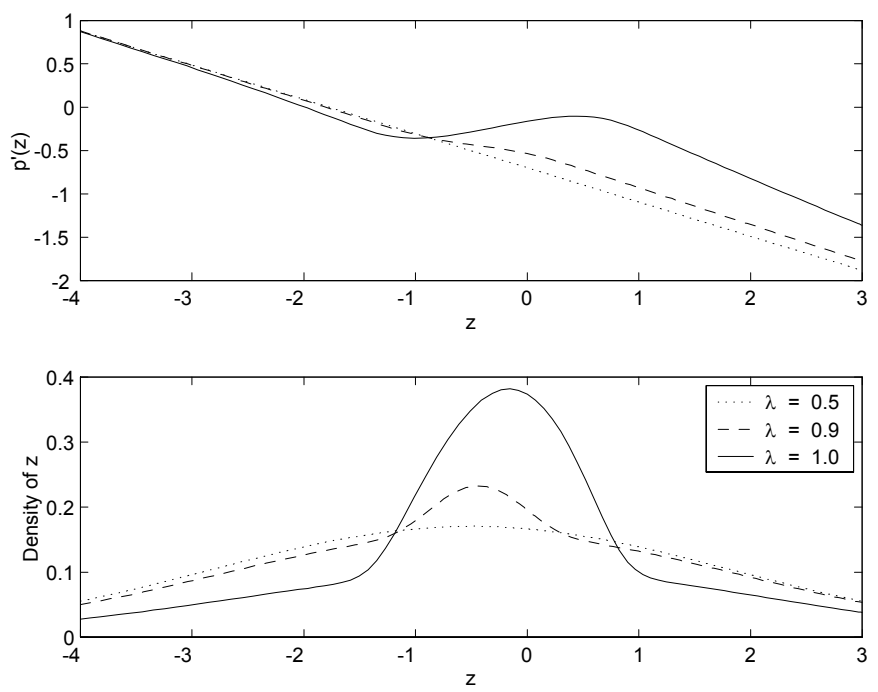

Figure 2: Model 1, Specification 1: Curvature of price function
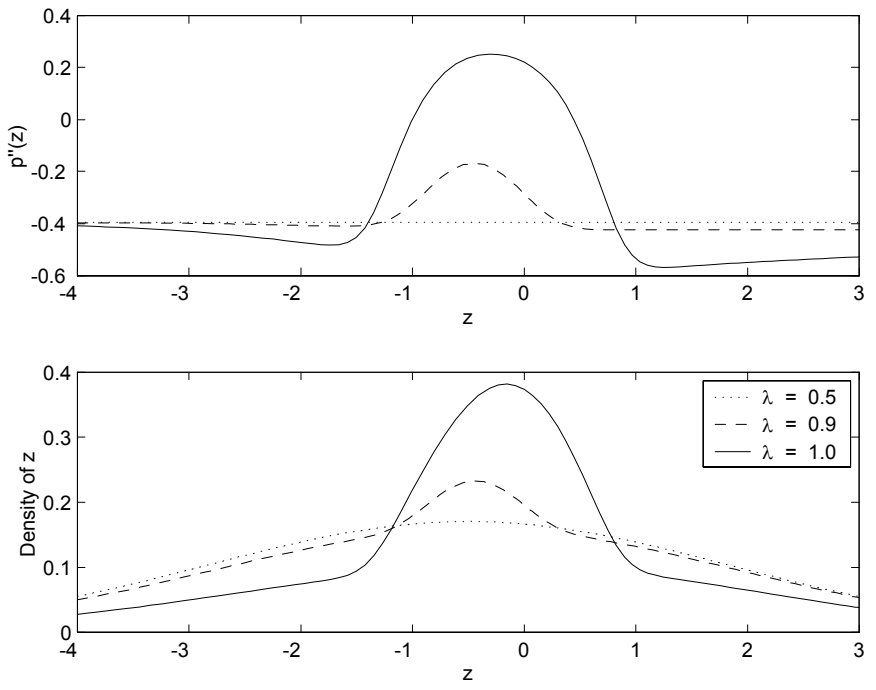
Figure 3: Model 1, Specification 2: Slope of price function
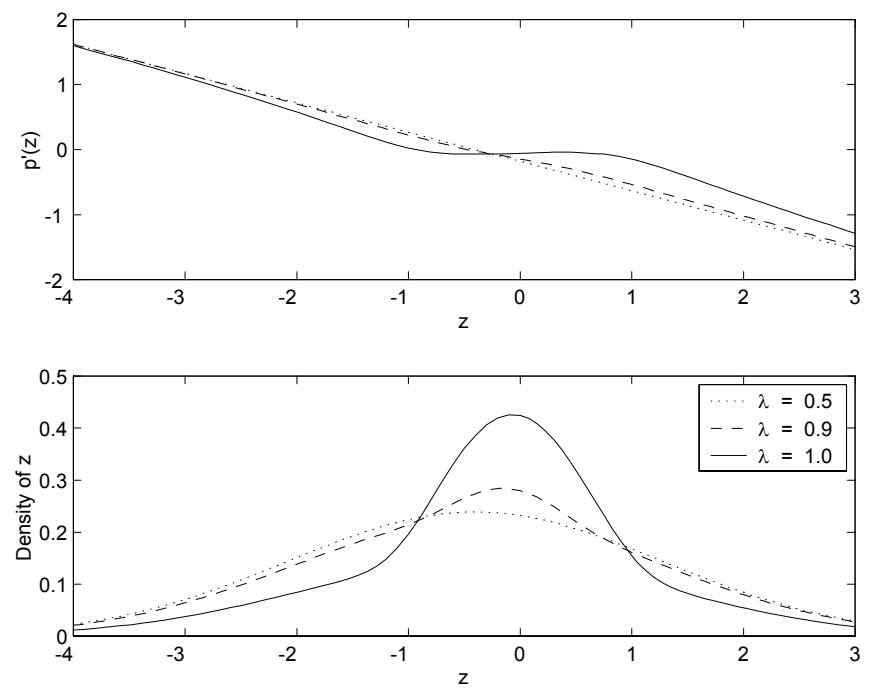

Figure 4: Model 1, Specification 2: Curvature of price function
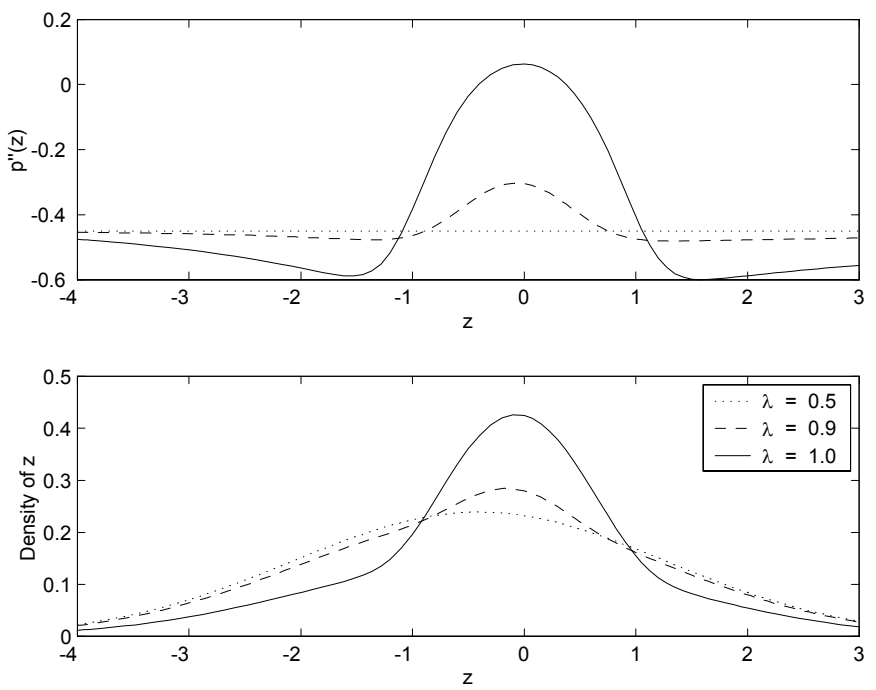
Figure 5: Model 1, Specification 3: Slope of price function
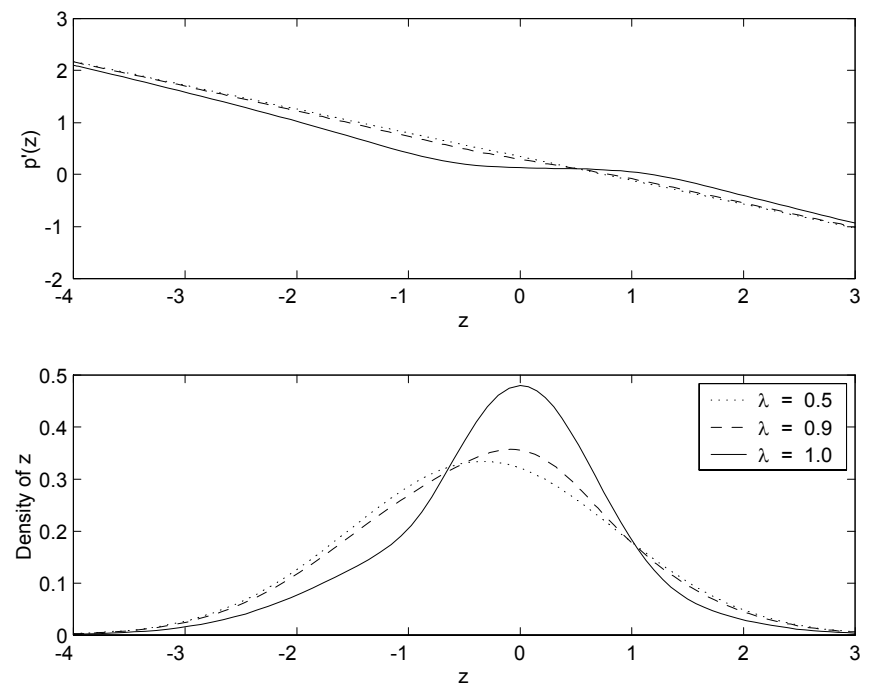

Figure 6: Model 1, Specification 3: Curvature of price function
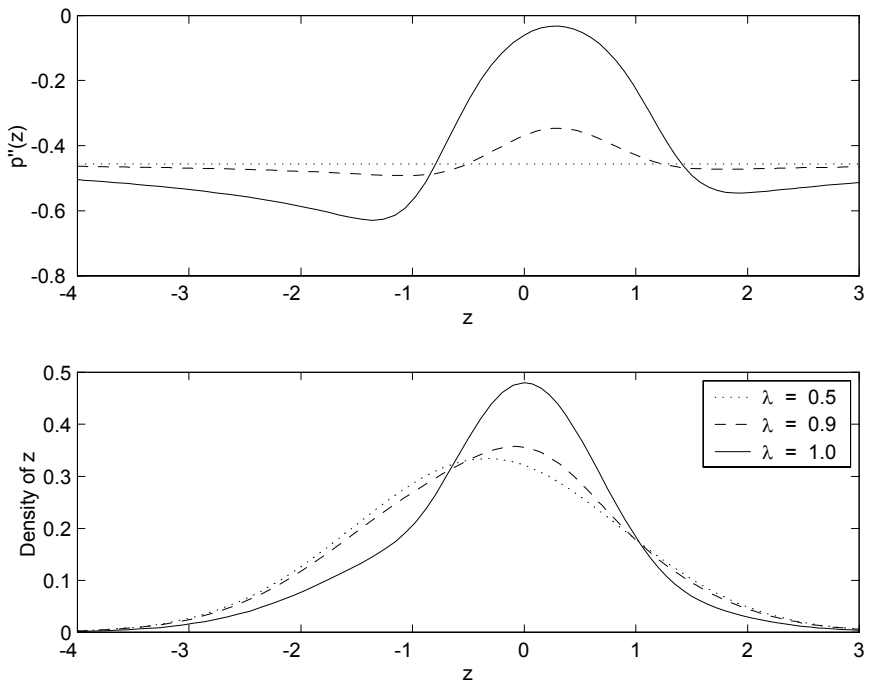
Figure 7: Model 1, Specification 4: Slope of price function
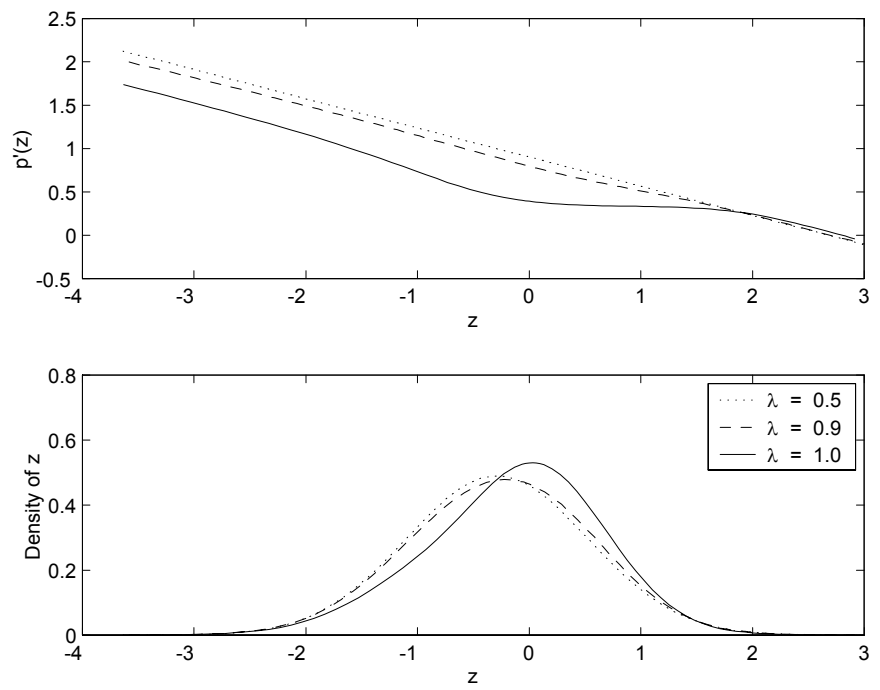

Figure 8: Model 1, Specification 4: Curvature of price function
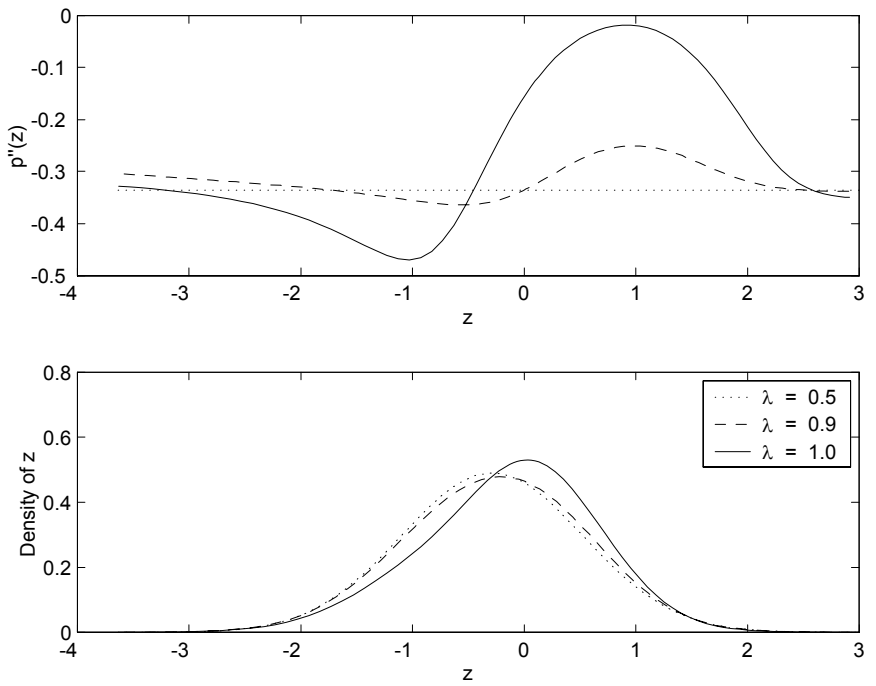
Figure 9: Model 1, Specification 5: Slope of price function
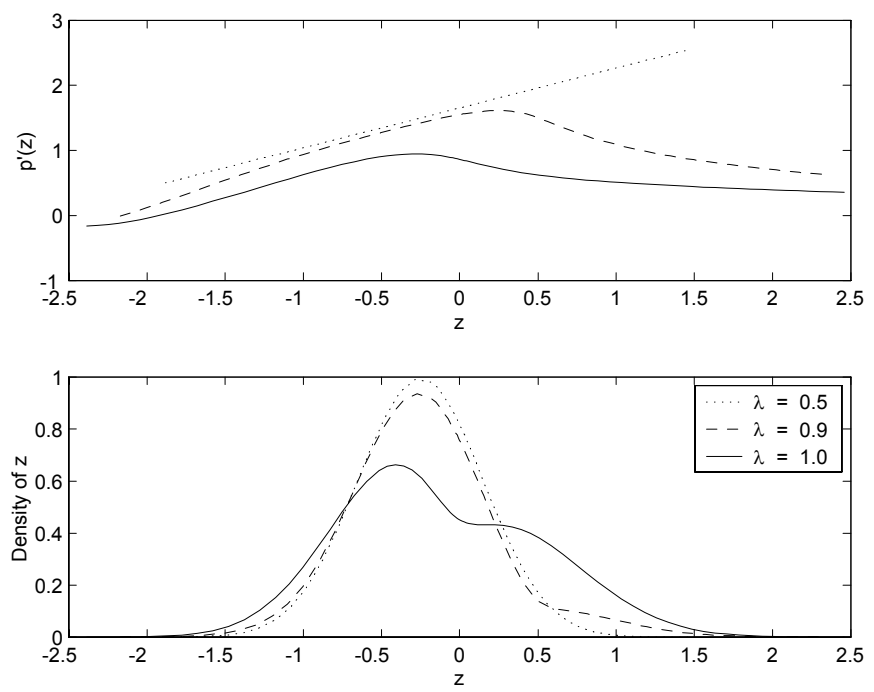

Figure 10: Model 1, Specification 5: Curvature of price function
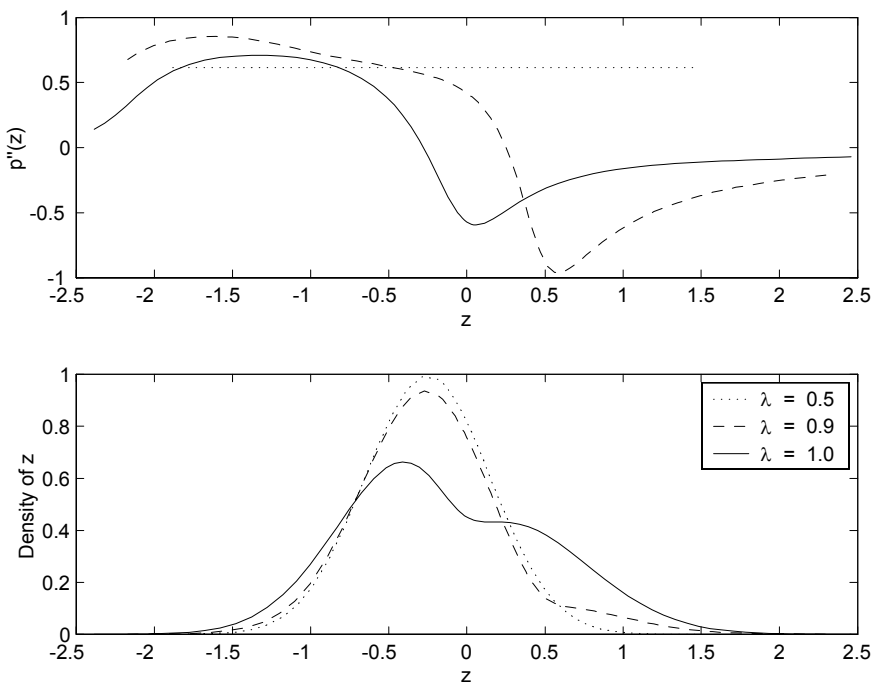
Figure 11: Model 1, Specification 1: Slope of price function
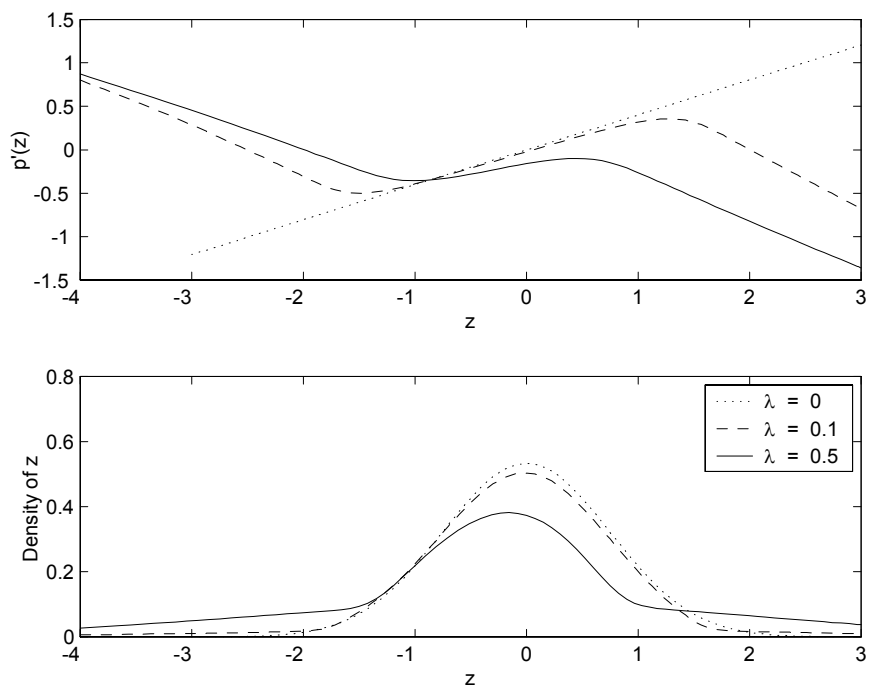

Figure 12: Model 1, Specification 1: Curvature of price function
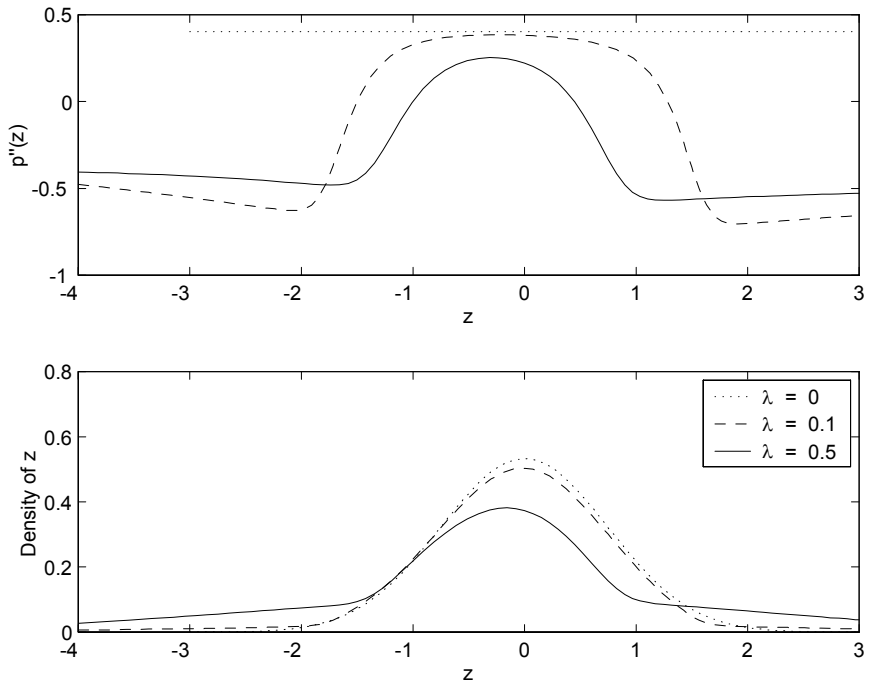
Figure 13: Model 1, Specification 2: Slope of price function
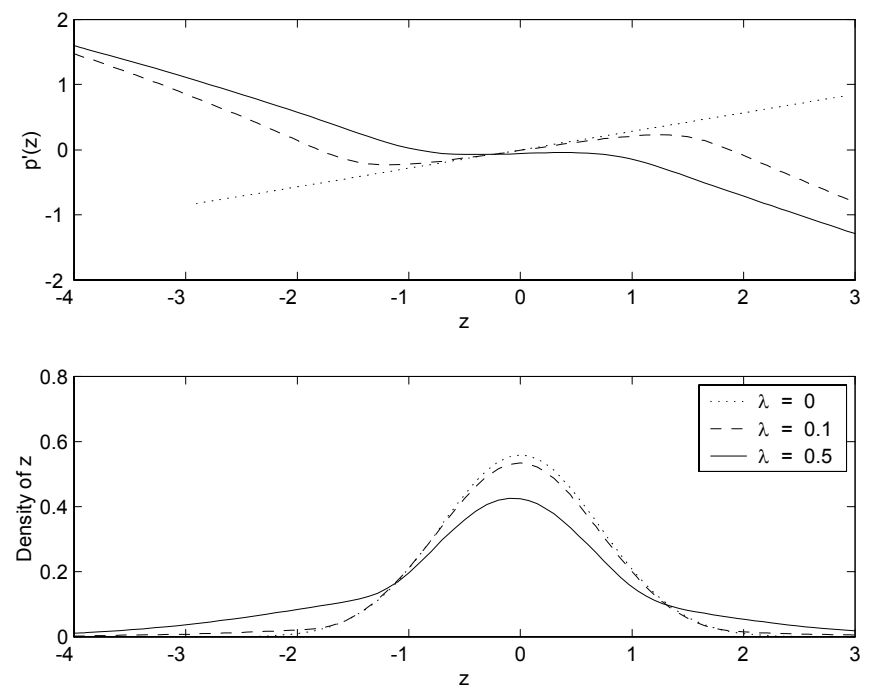

Figure 14: Model 1, Specification 2: Curvature of price function
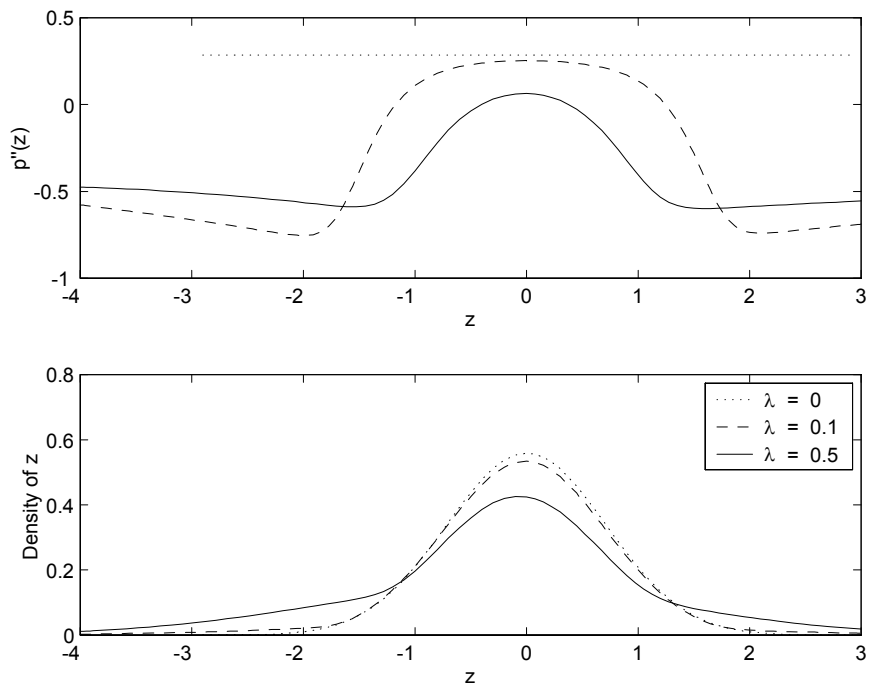
Figure 15: Model 1, Specification 3: Slope of price function
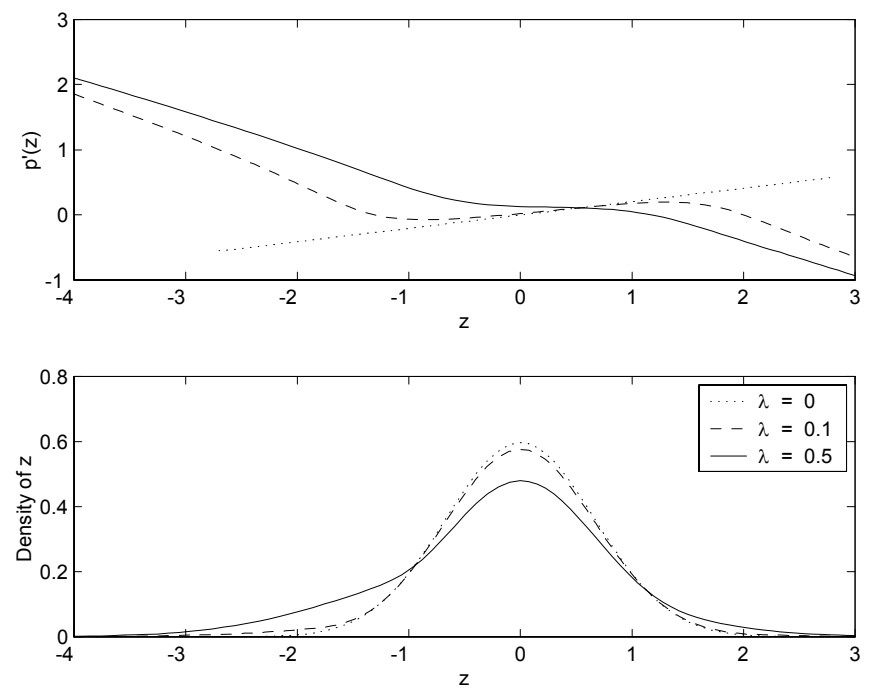

Figure 16: Model 1, Specification 3: Curvature of price function
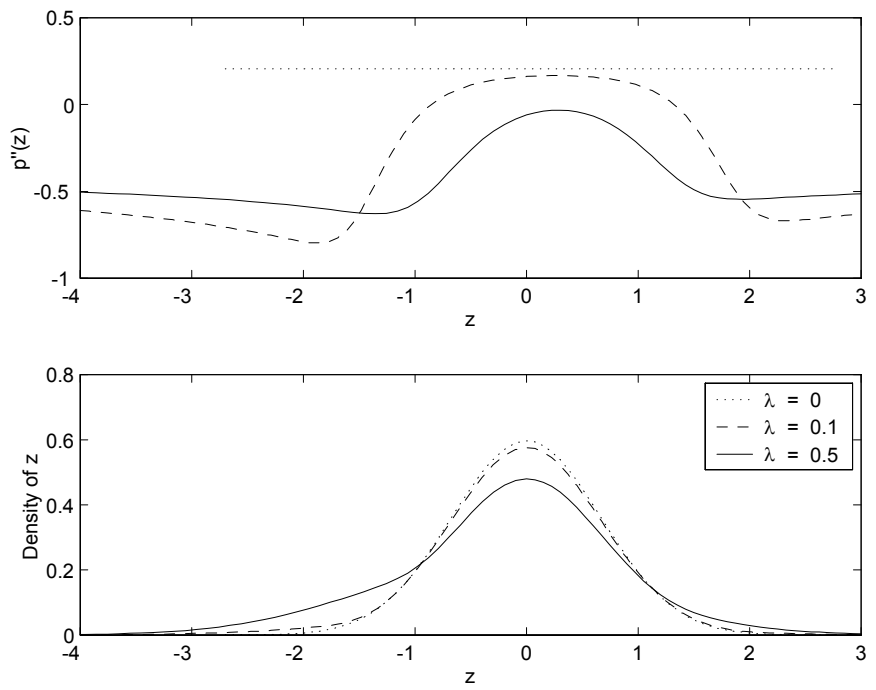
Figure 17: Model 1, Specification 4: Slope of price function
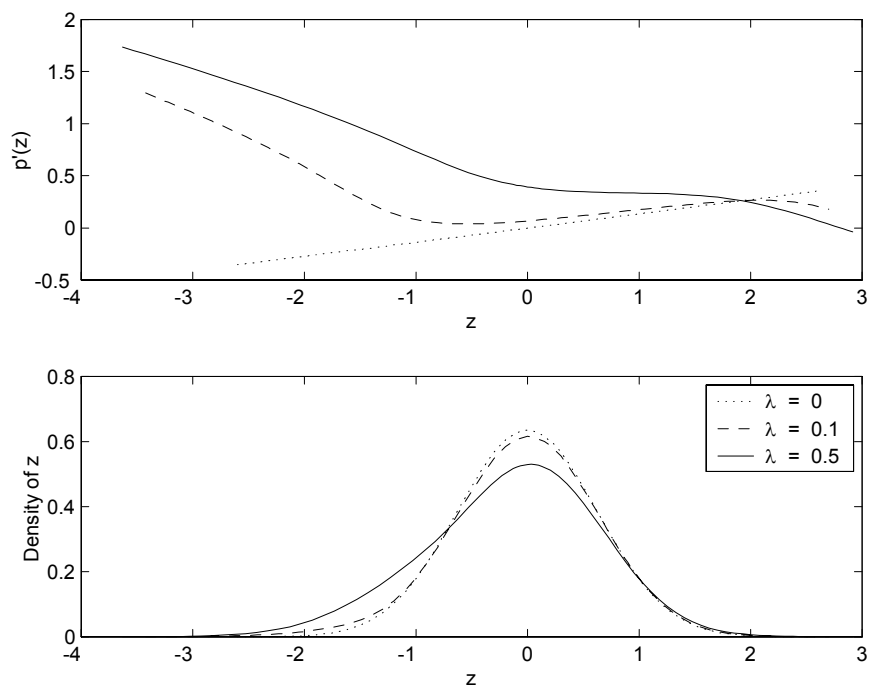

Figure 18: Model 1, Specification 4: Curvature of price function
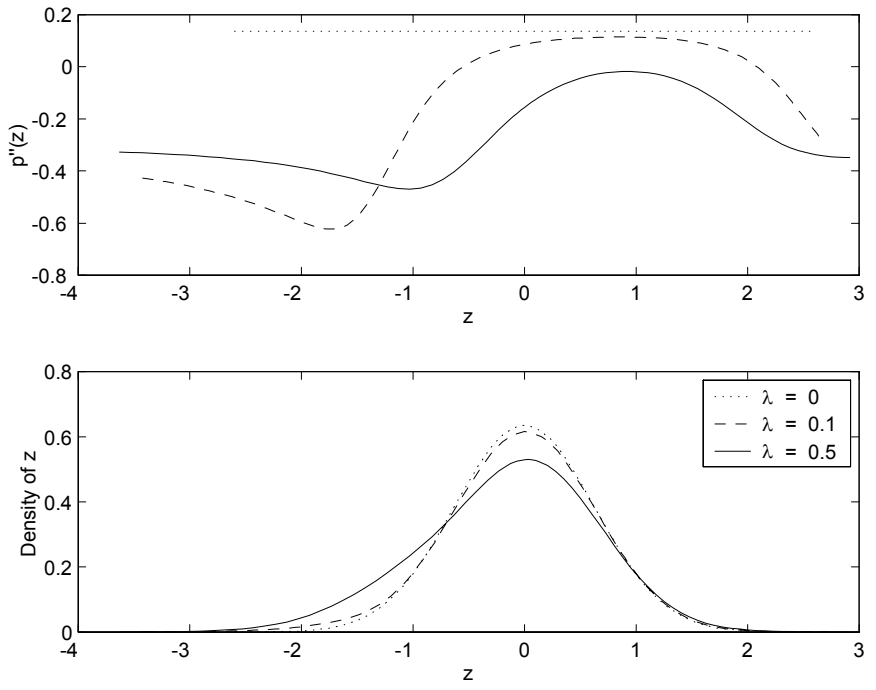
Figure 19: Model 1, Specification 5: Slope of price function
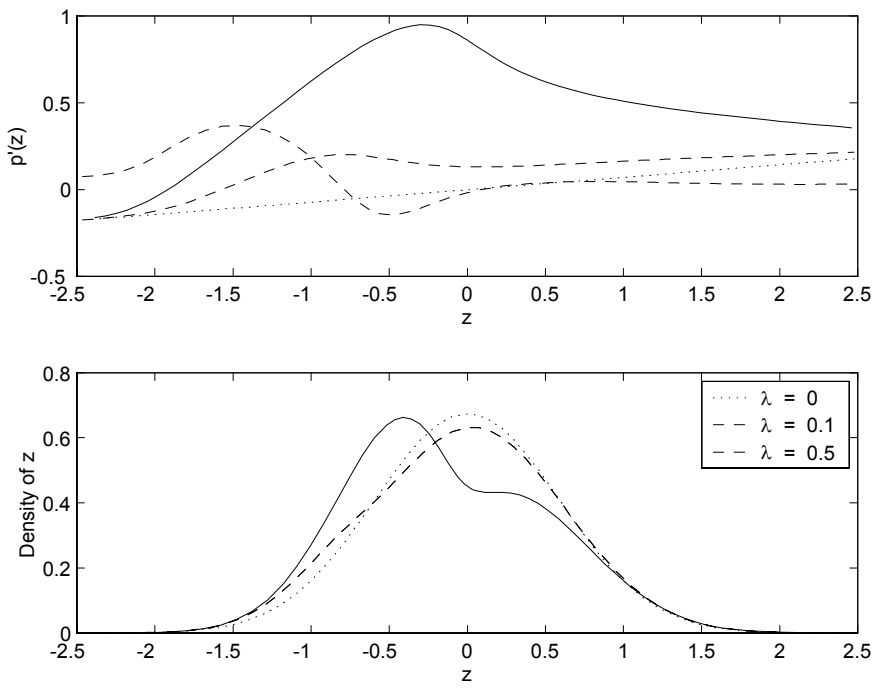

Figure 20: Model 1, Specification 5: Curvature of price function
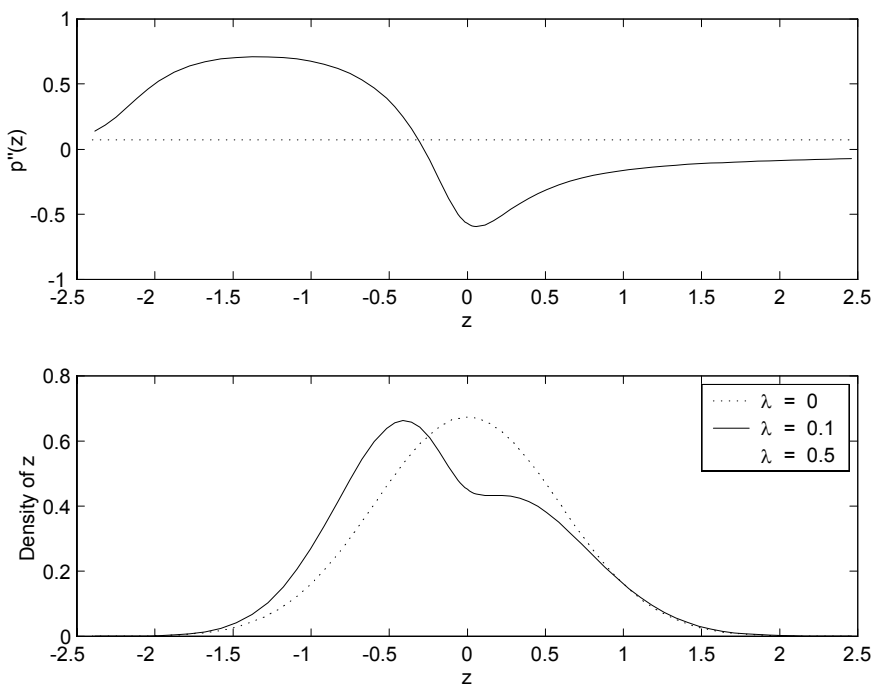
Figure 21: Model 2, Specification 1: Slope of price function
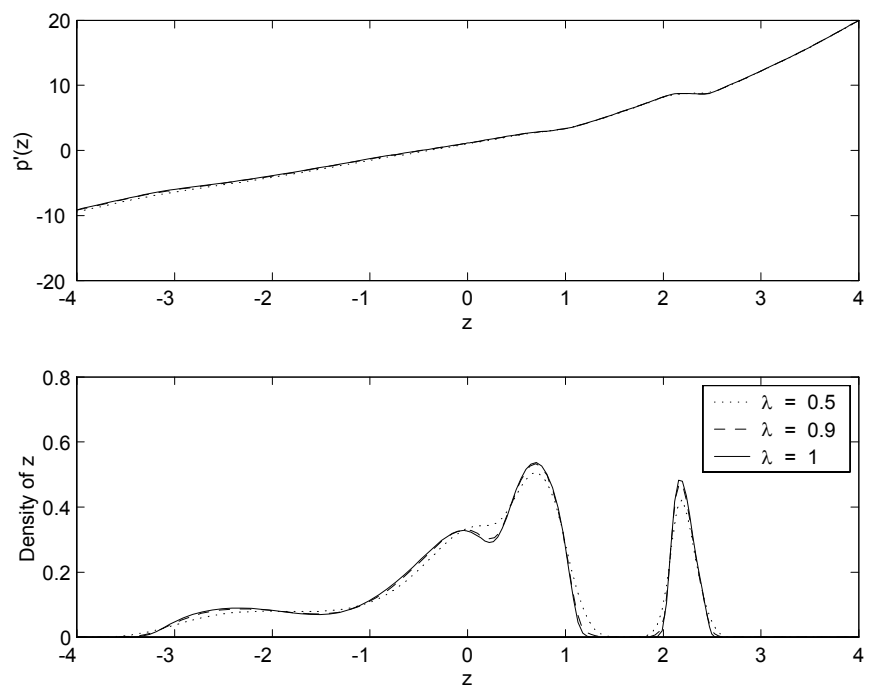

Figure 22: Model 2, Specification 1: Curvature of price function
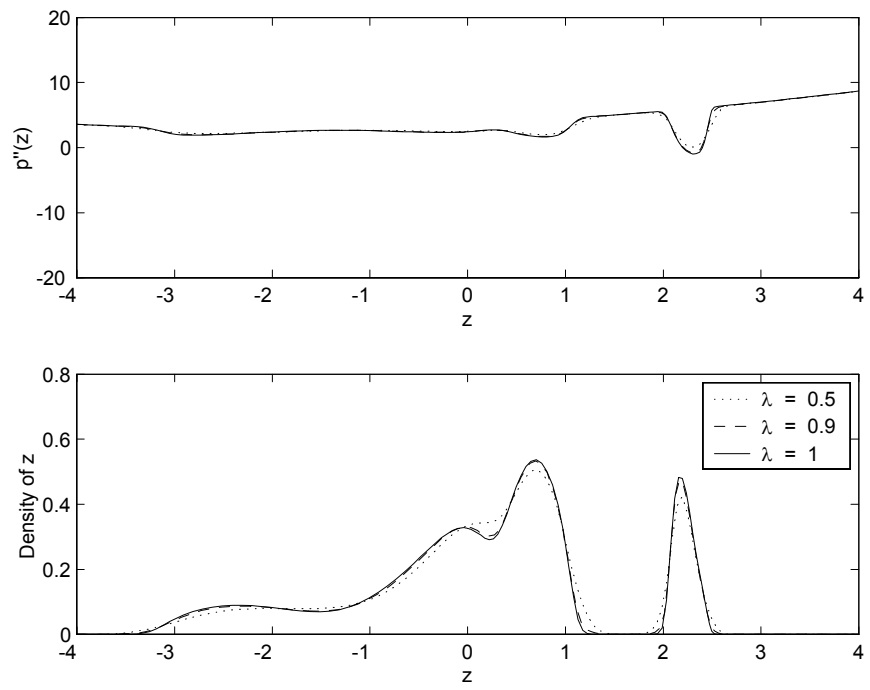
Figure 23: Model 2, Specification 2: Slope of price function
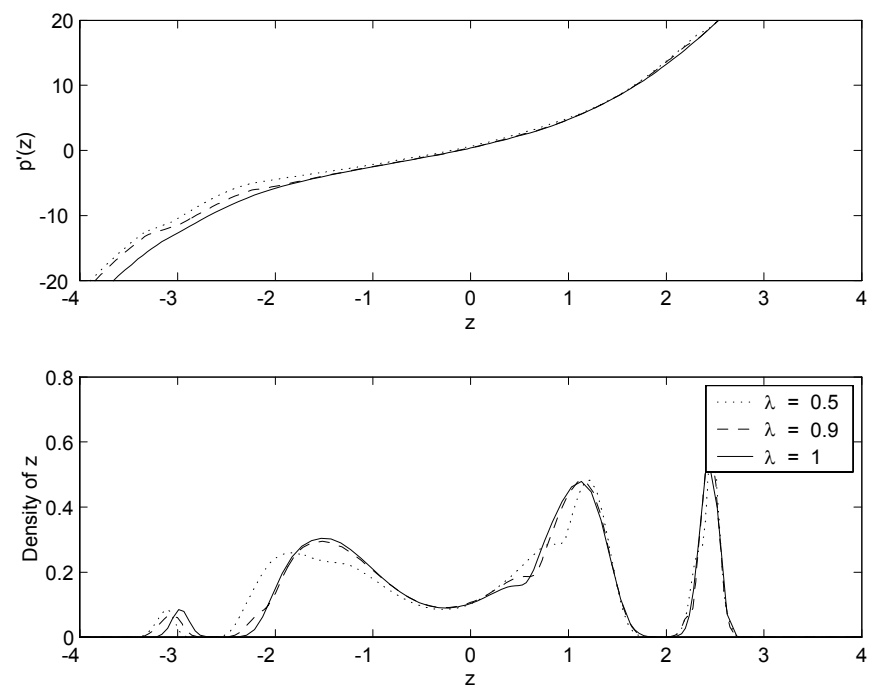

Figure 24: Model 2, Specification 2: Curvature of price function
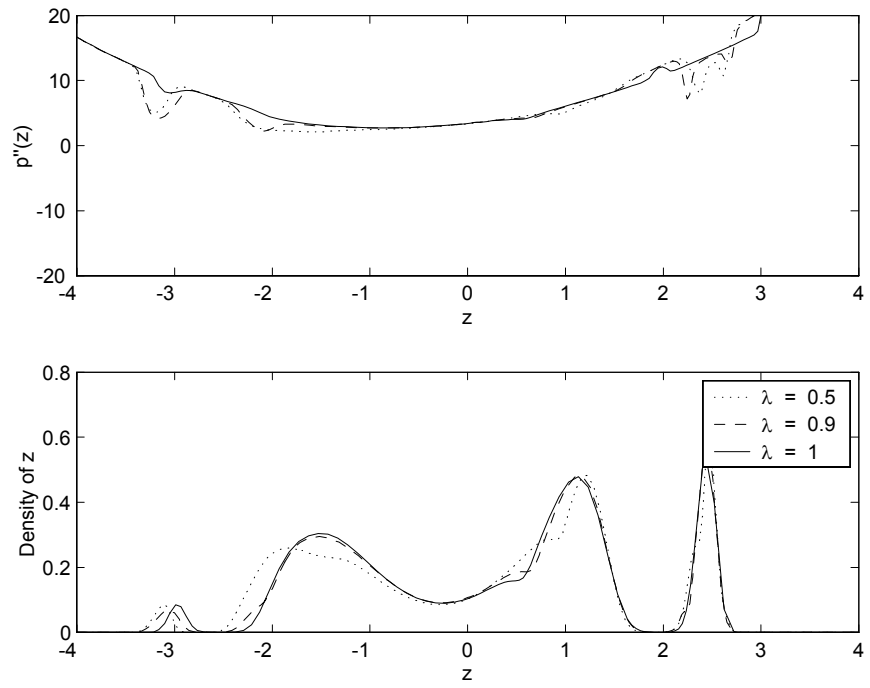
Figure 25: Model 2, Specification 3: Slope of price function
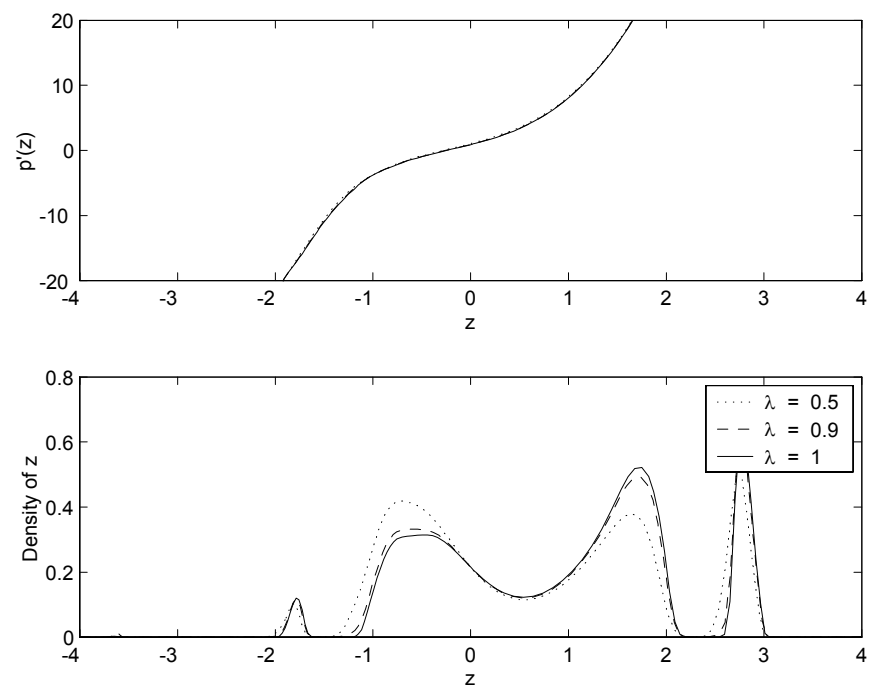

Figure 26: Model 2, Specification 3: Curvature of price function
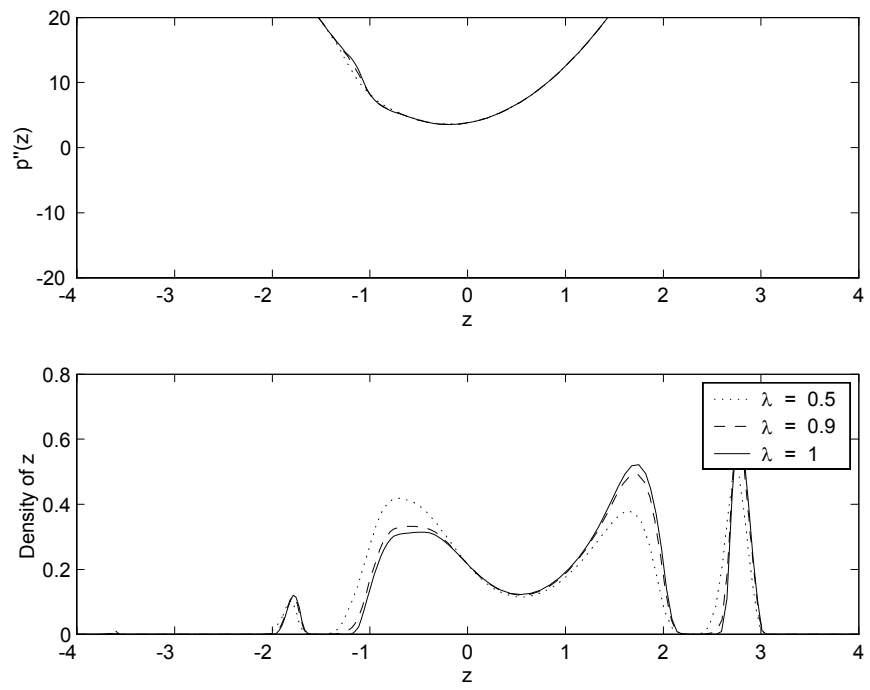
Figure 27: Model 2, Specification 4: Slope of price function
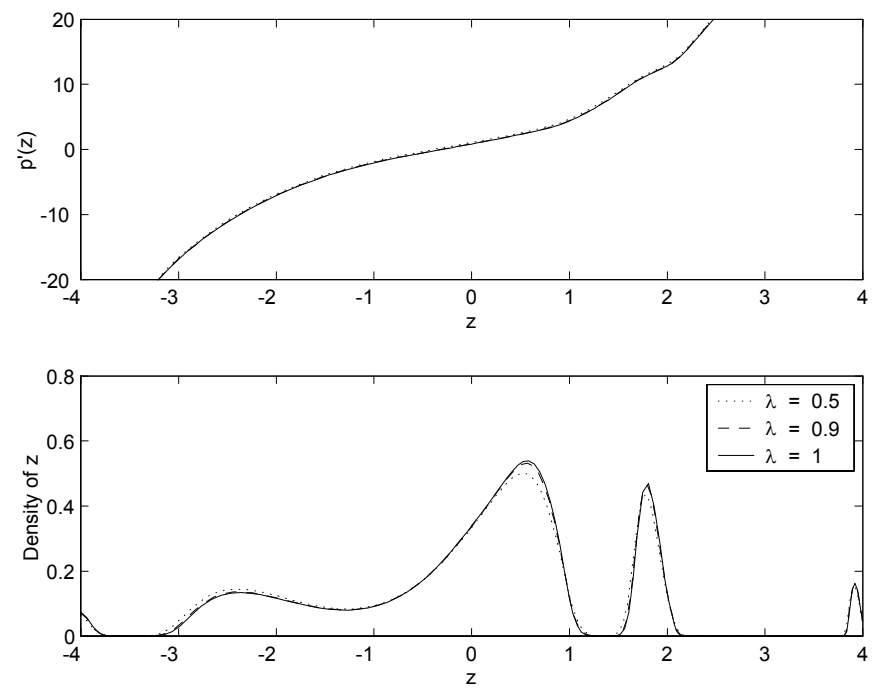

Figure 28: Model 2, Specification 4: Curvature of price function
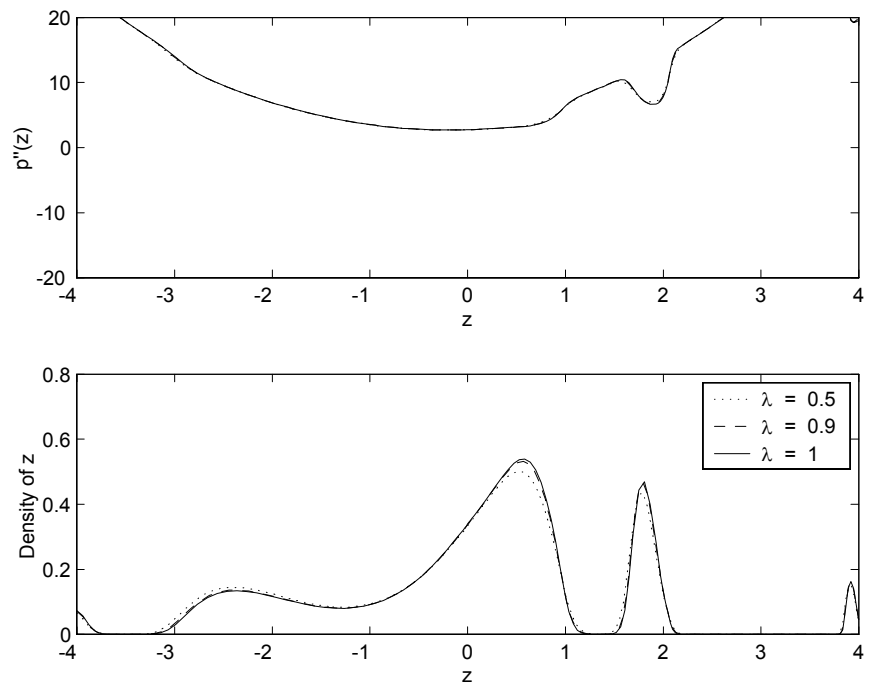
Figure 29: Model 2, Specification 5: Slope of price function
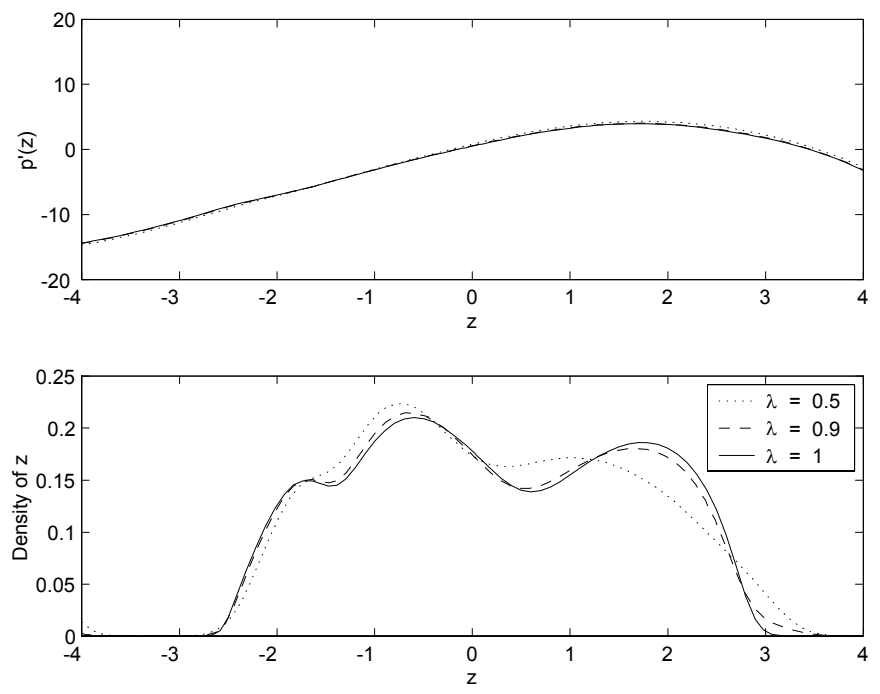

Figure 30: Model 2, Specification 5: Curvature of price function
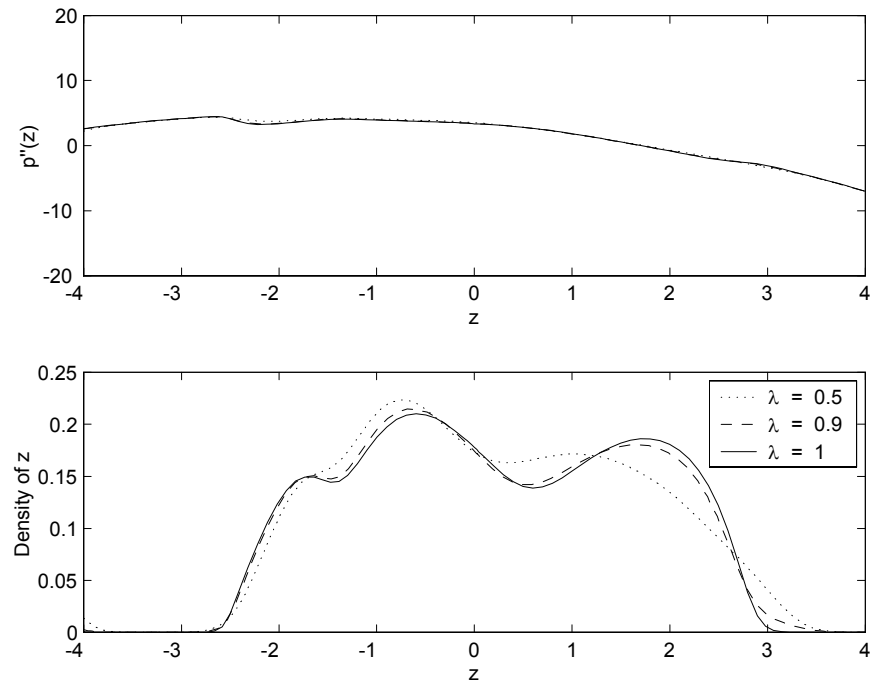
Figure 31: Model 3: Slope of price function

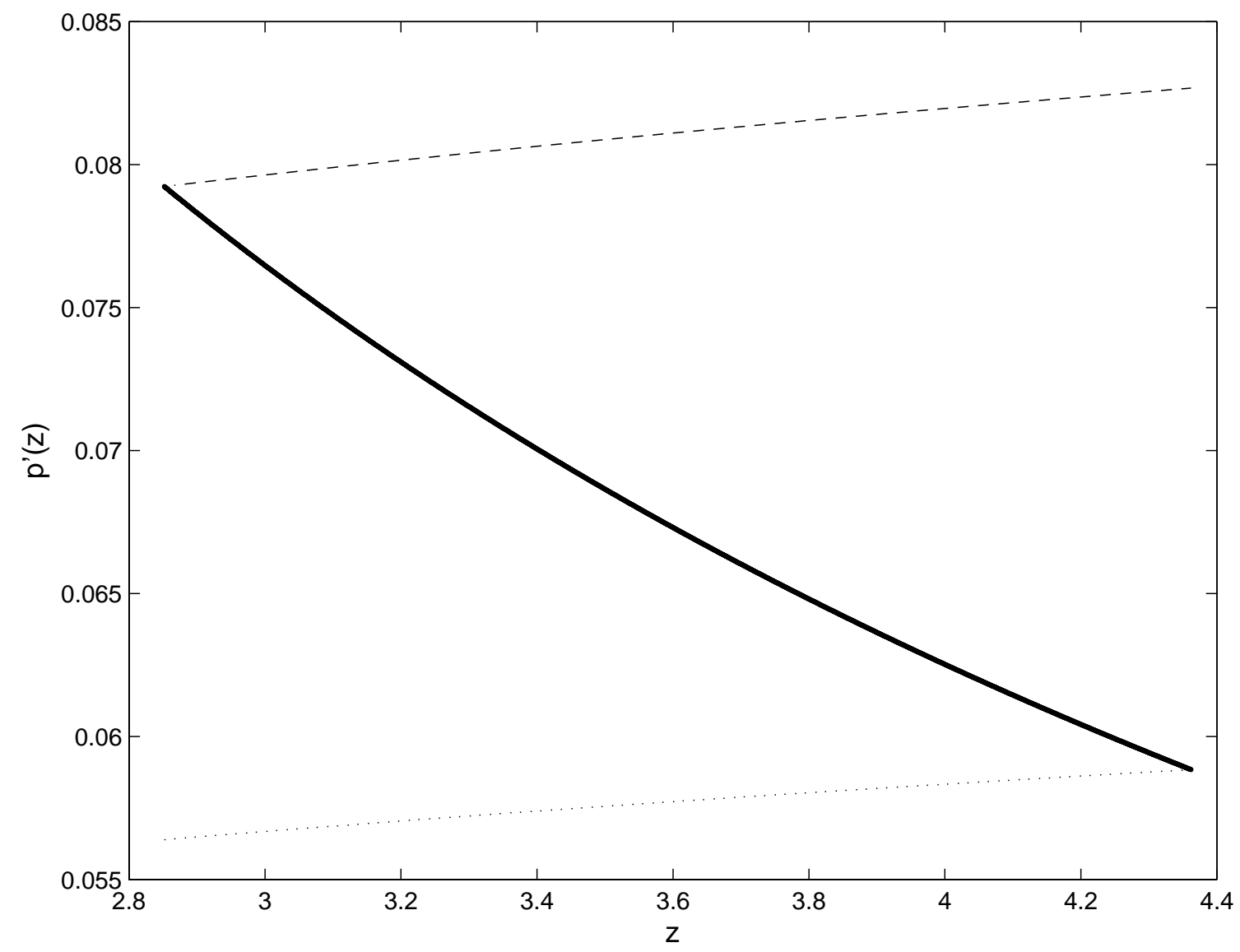



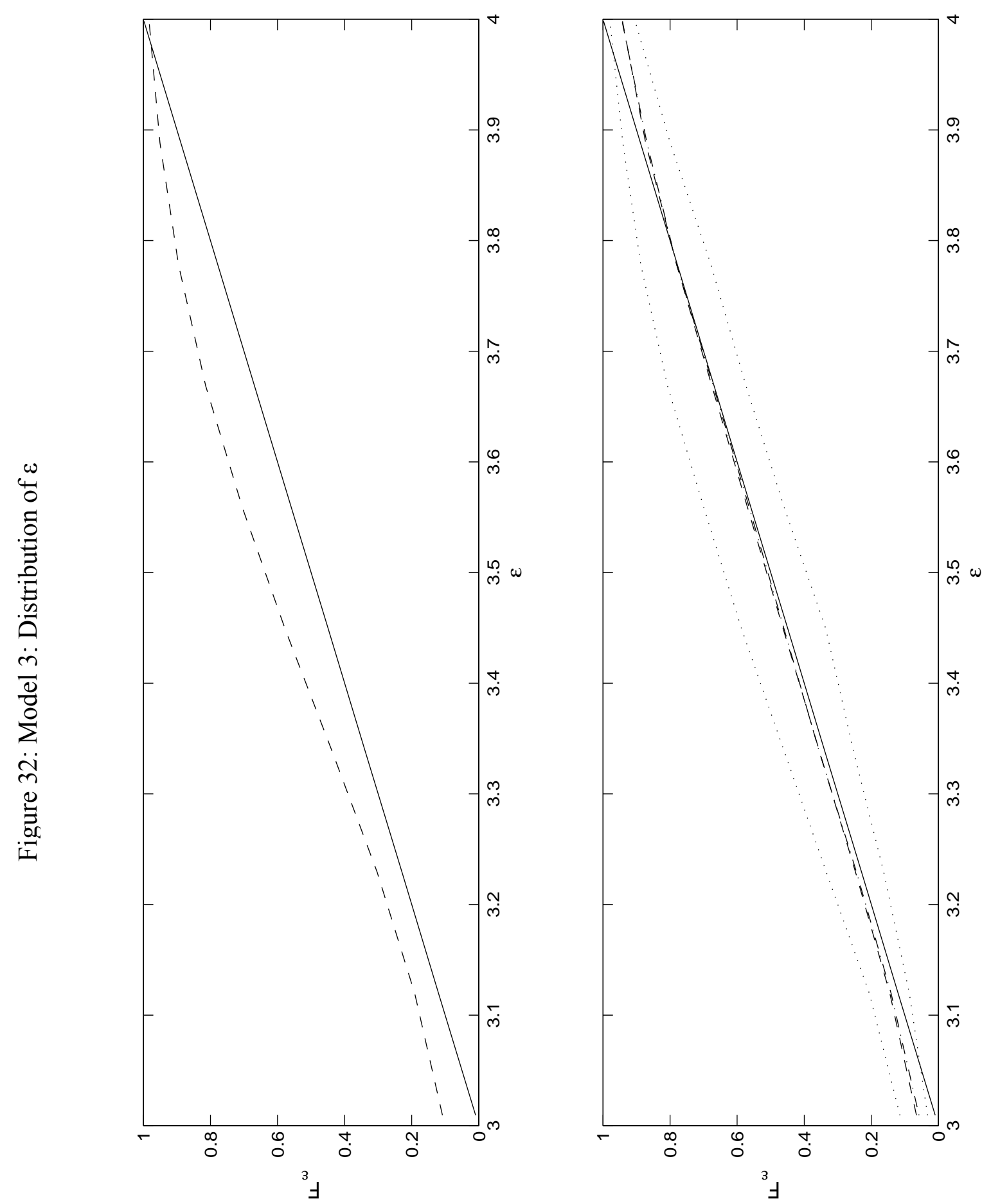

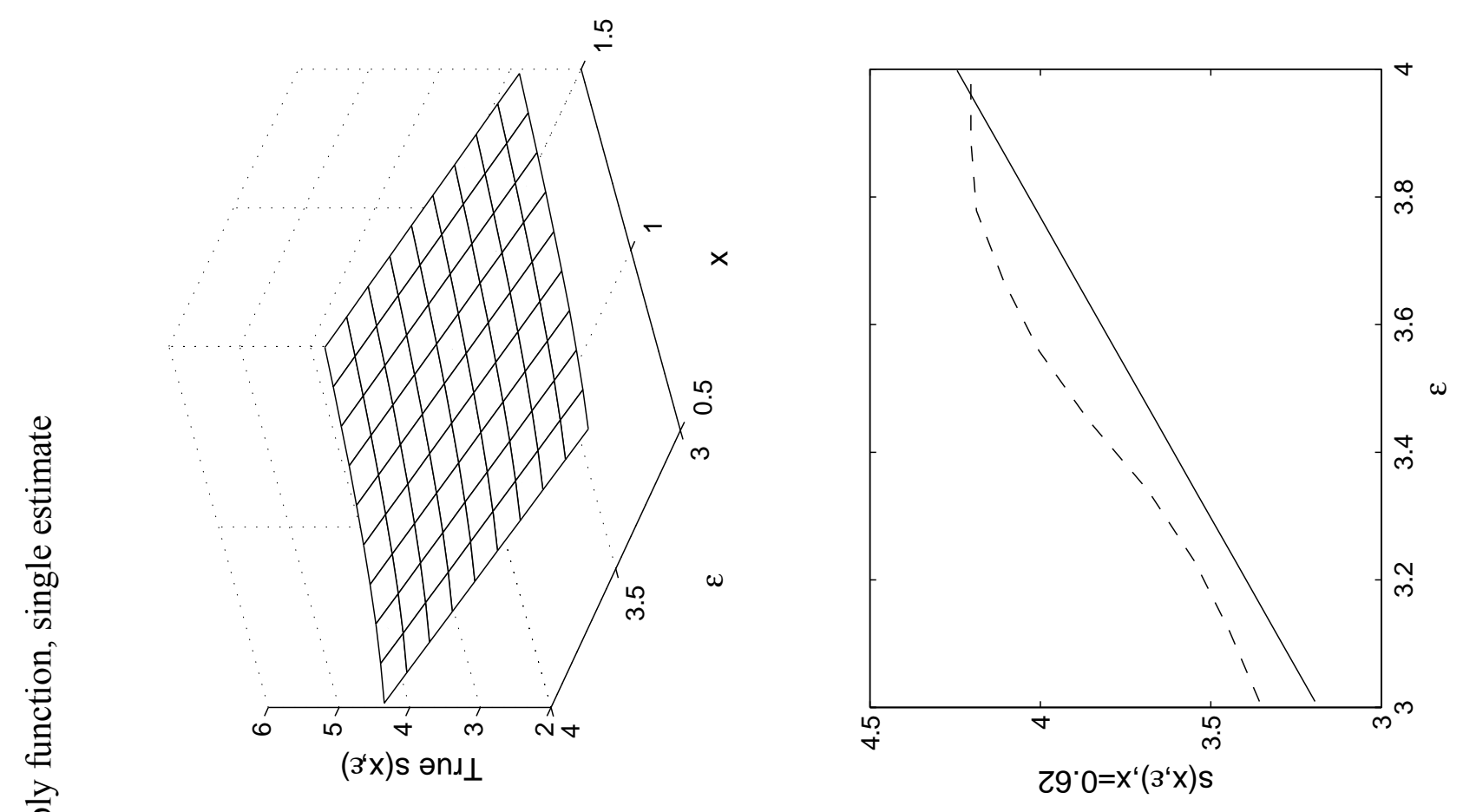

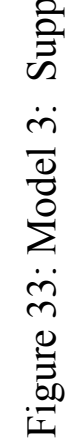



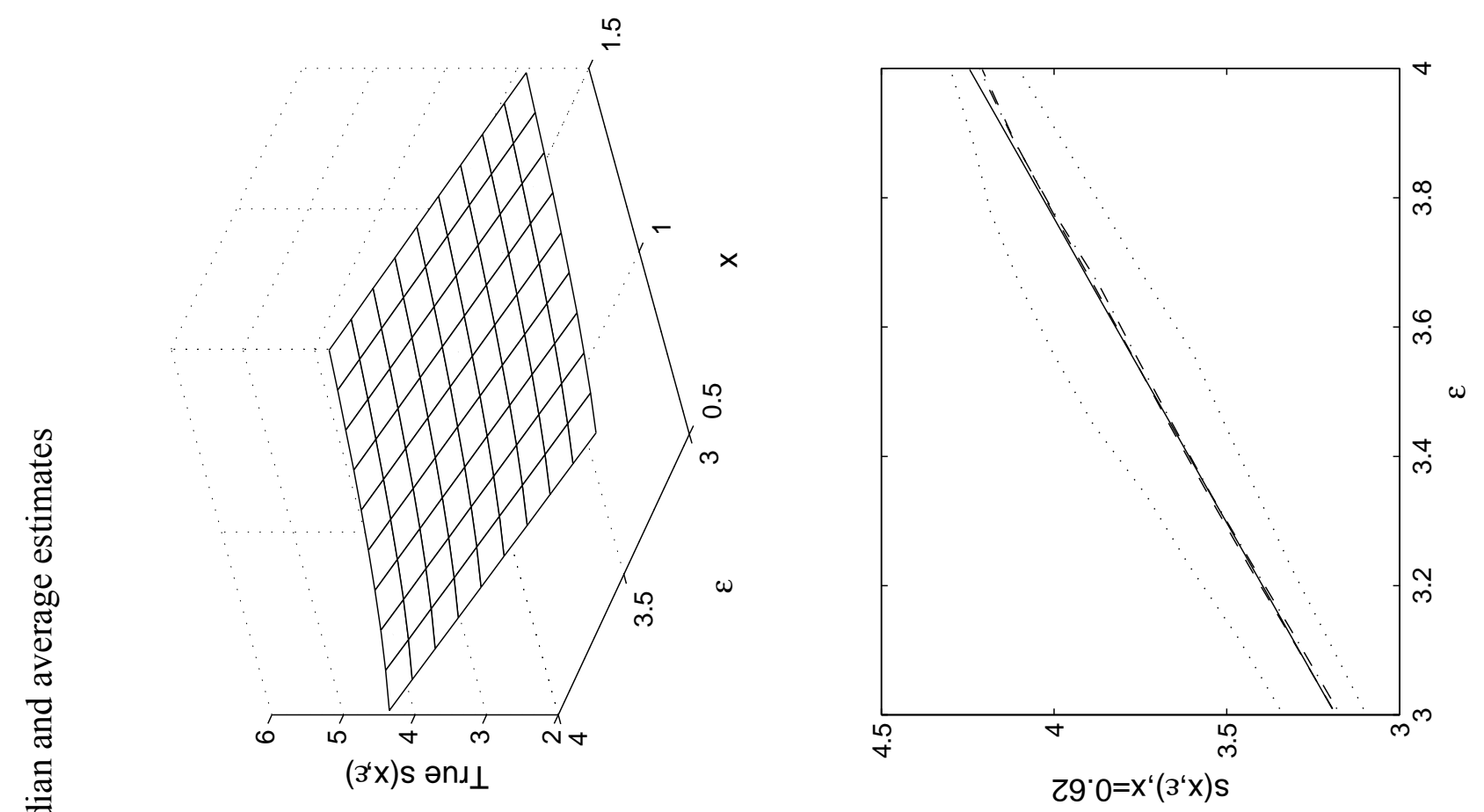

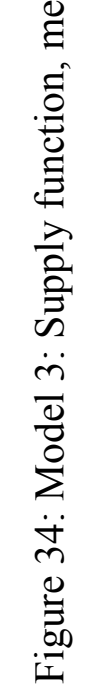




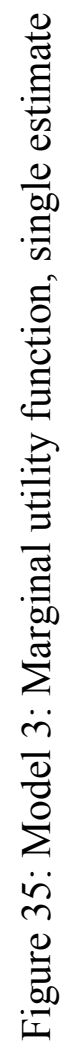
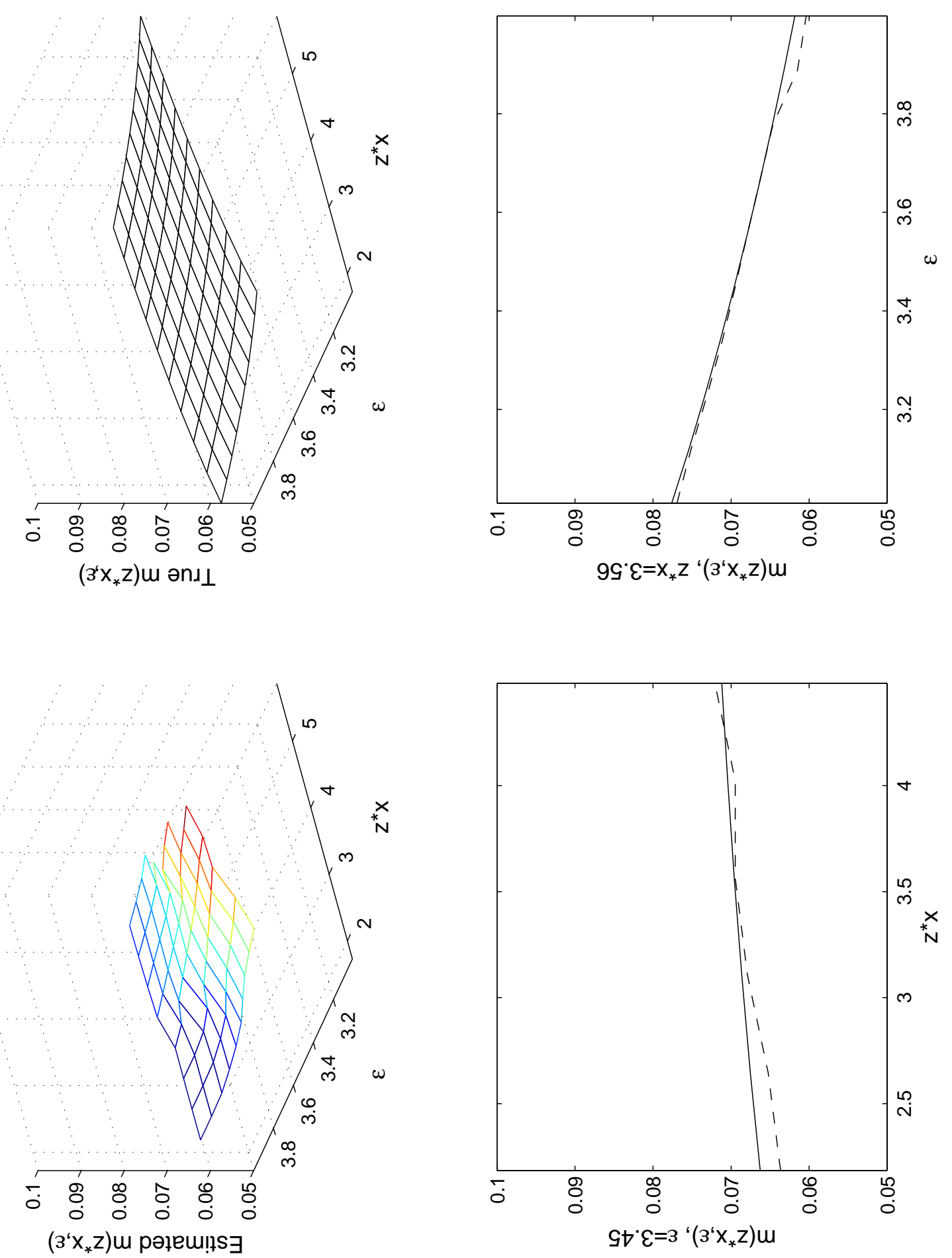

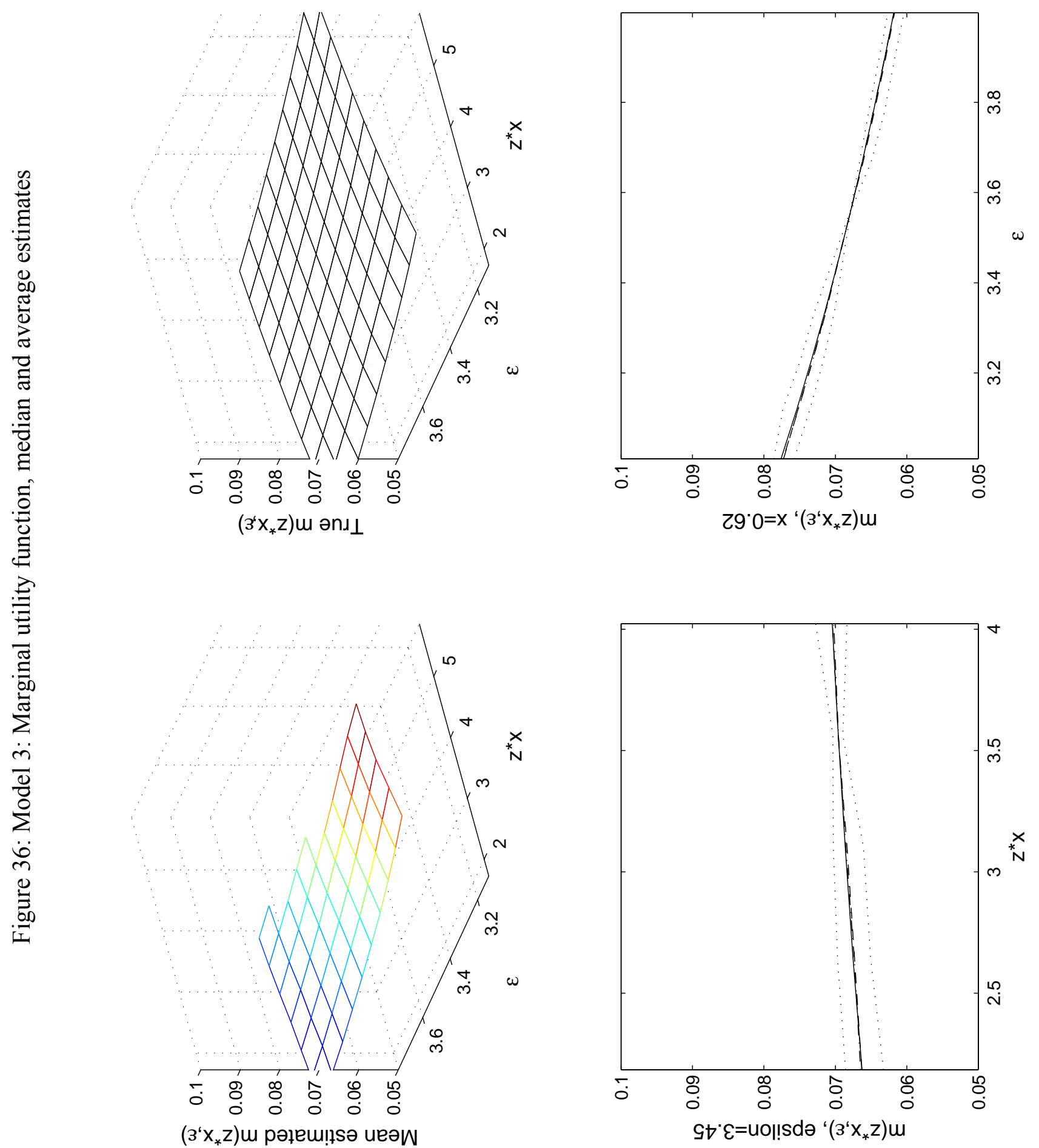


\section{A Tables}

Table A1:

Model 1 functional forms

\begin{tabular}{|c|c|c|c|}
\hline Firm & Technology & $\Gamma(z, y, \eta)$ & $\left(\nu_{0}+\nu_{1} y+\eta\right) z-\frac{B z^{2}}{2}$ \\
\hline & Density of $y$ & $f_{y}(y)$ & $\sum_{i=1}^{M_{y}} \lambda_{y i} \phi\left(y, \mu_{y i}, \Sigma_{y i}\right)$ \\
\hline & Density of $\eta$ & $f_{\eta}(\eta)$ & $\sum_{i=1}^{M_{\eta}} \lambda_{\eta i} \phi\left(\eta, \mu_{\eta i}, \Sigma_{\eta i}\right)$ \\
\hline Worker & Utility & $U(z, x, \varepsilon)$ & $\left(\theta_{0}+\theta_{1} x+\varepsilon\right) z-\frac{A z^{2}}{2}$ \\
\hline & Density of $x$ & $f_{x}(x)$ & $\sum_{i=1}^{M_{x}} \lambda_{x i} \phi\left(x, \mu_{x i}, \Sigma_{x i}\right)$ \\
\hline & Density of $\varepsilon$ & $f_{\varepsilon}(\varepsilon)$ & $\sum_{i=1}^{M_{\varepsilon}} \lambda_{\varepsilon i} \phi\left(\varepsilon, \mu_{\varepsilon i}, \Sigma_{\varepsilon i}\right)$ \\
\hline
\end{tabular}

$(z, \eta, \varepsilon)$ are all scalars. $y$ is of dimension $n_{y}$ and $x$ is of dimension $n_{x}$. The parameters $\left(B, \nu_{0}, \nu_{1}\right)$ and $\left(A, \theta_{0}, \theta_{1}\right)$ are common across all firms and workers respectively. 
:

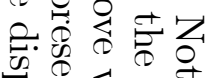

류류.

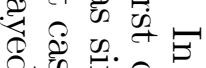

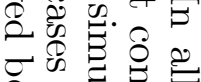

运㐘

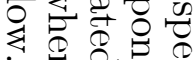

एव $\stackrel{9}{\circ}$

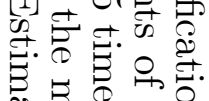

范怘点

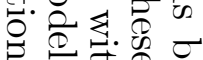

-

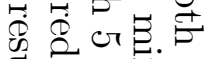

局

क 峞节

थ०त की

- $\stackrel{2}{2} x^{m}$

के

司若气

胥。品

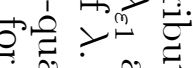

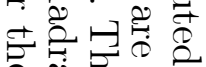

के कृ

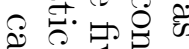

की

×

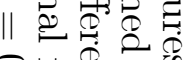

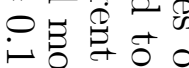

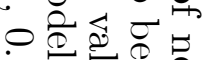

نे

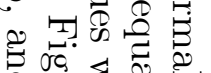

记

○ी०

的工

莣.

家ㅇㅇㅇ 0

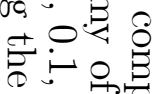

응무유

प्र

0

क

吉记

त ट्र

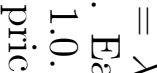

द्धे है के

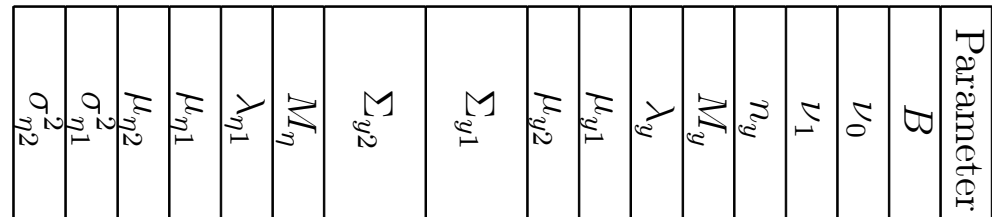

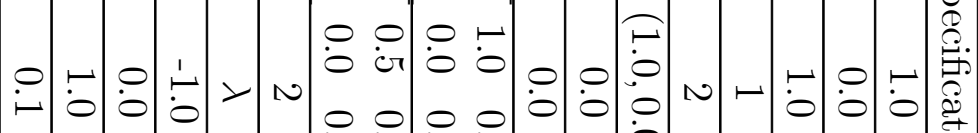

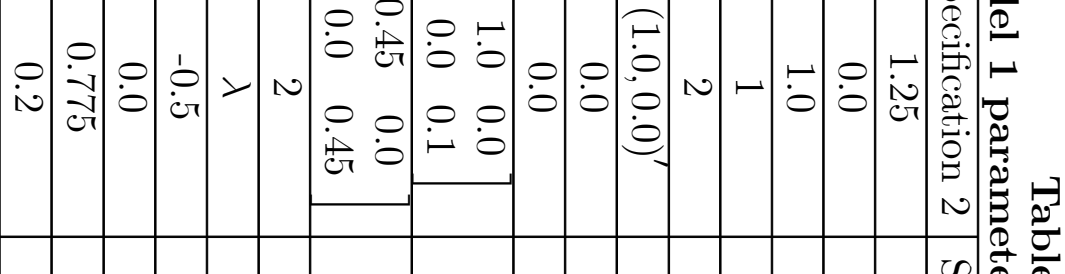

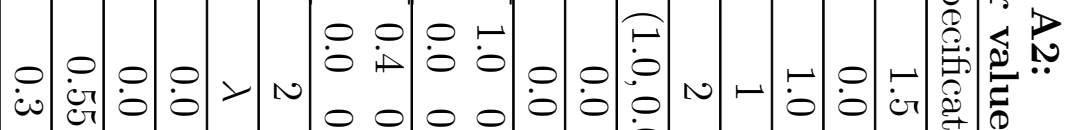

它定

节声吉

0 is

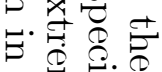

苟若

की

की की 
뉴유

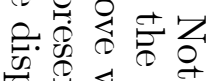

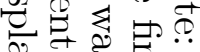

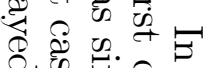

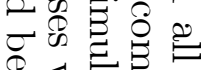

¿

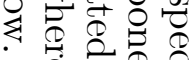

ज记蚂

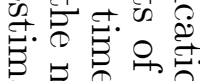

常.

욜요

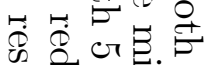

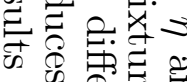

की

눈

की 0 过

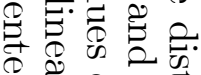

运禹

웅을

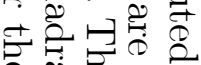

(2)

ช คิ

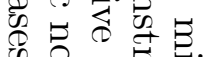

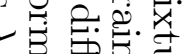

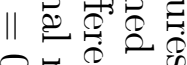

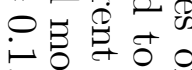

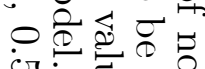
구

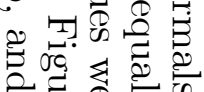

范舟

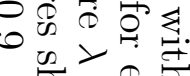
告车官

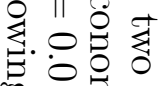

舟每

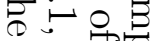

in 으용

당

D० 0 ?

우울.

氜

드욤

․․․

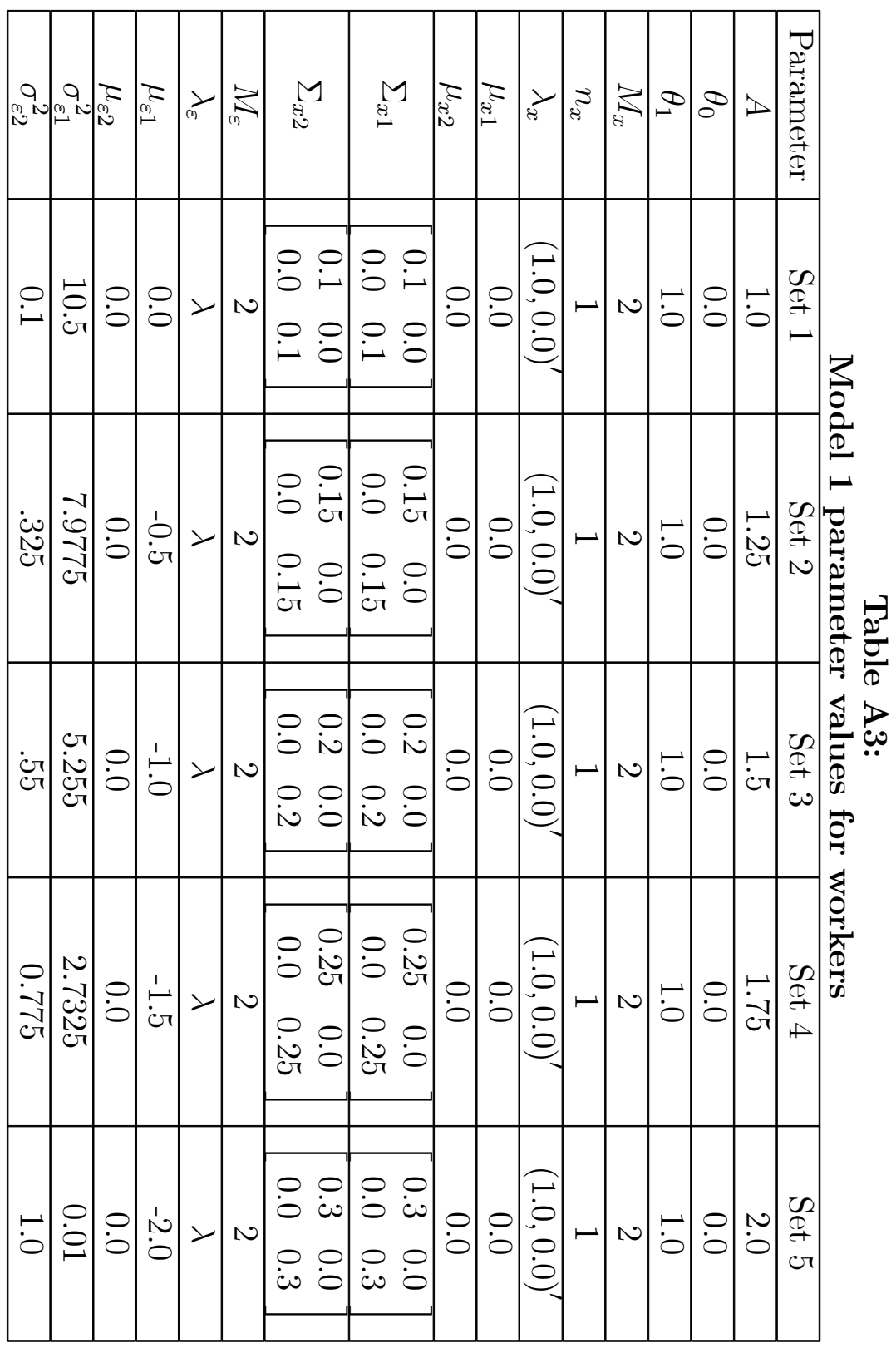

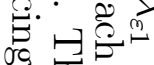

承。

苛志志

o d o

ㄱ.

苞苗

के

की 


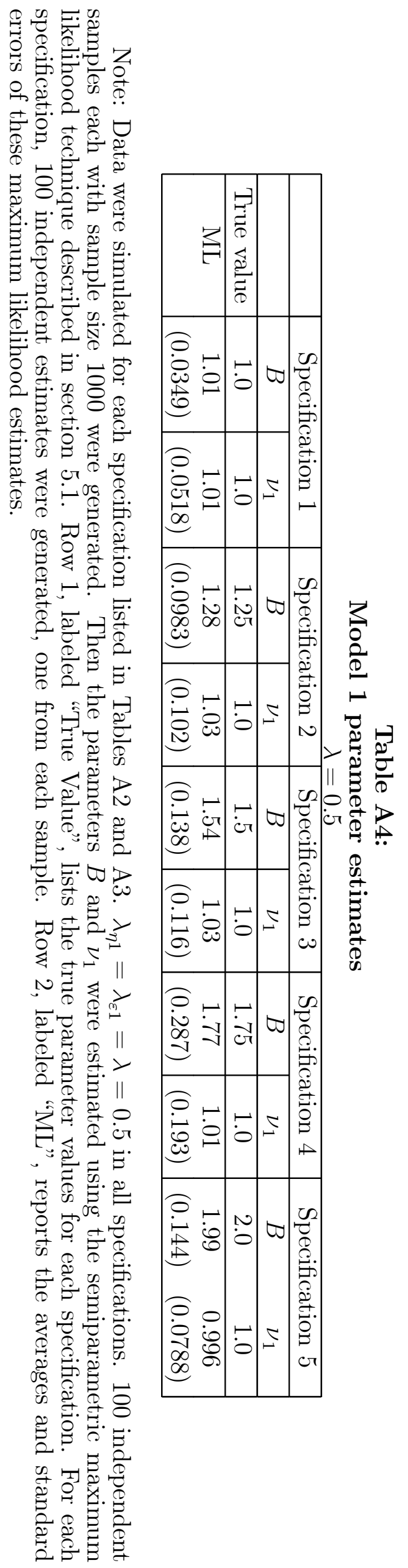




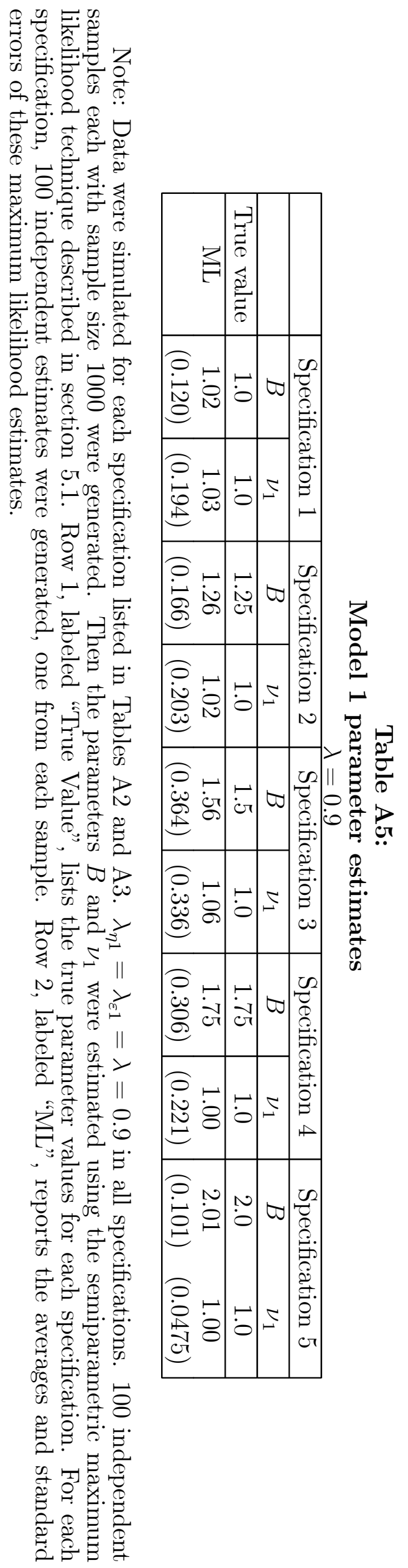




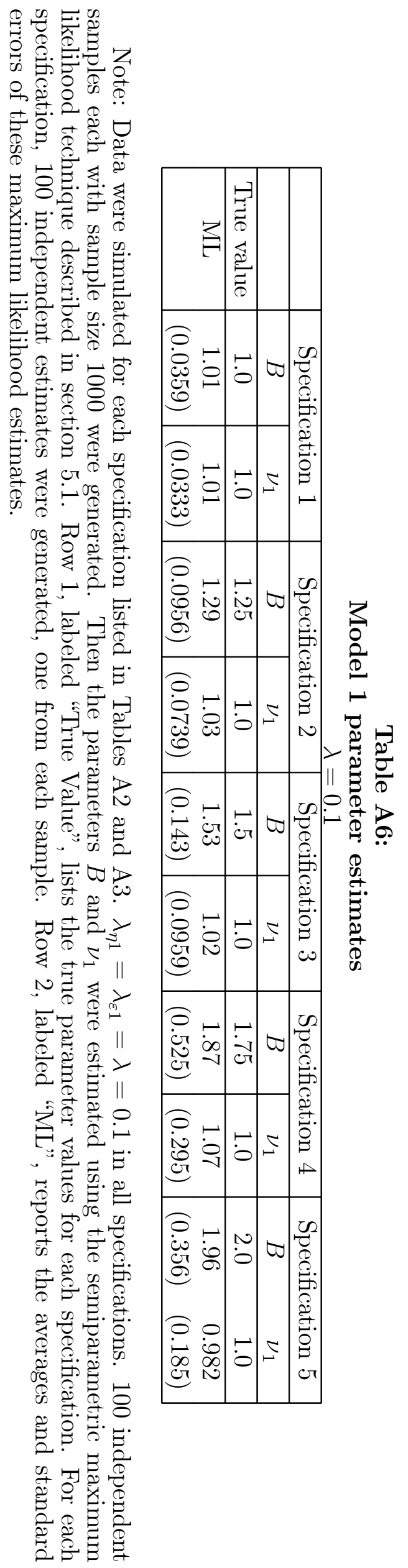




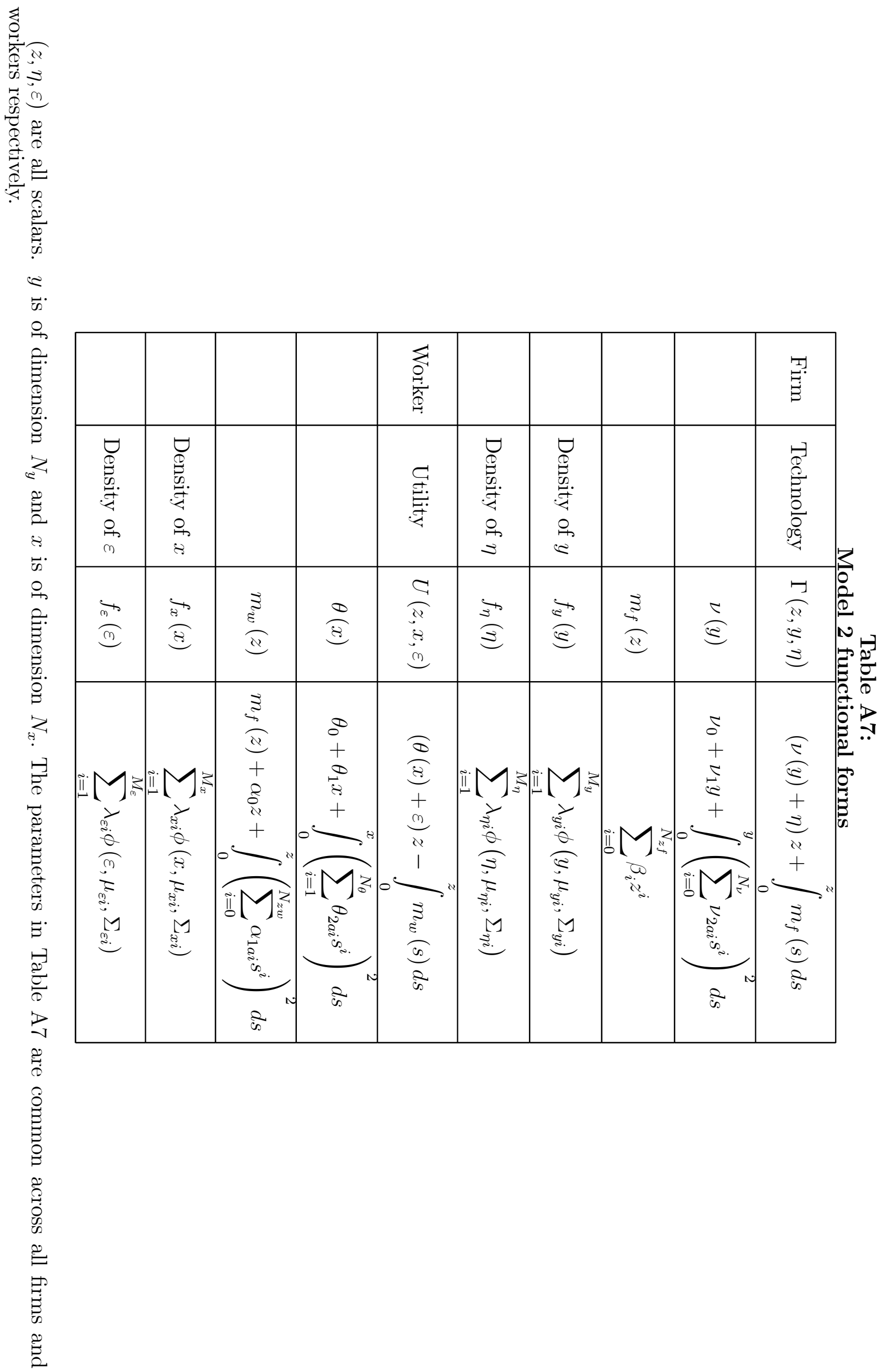




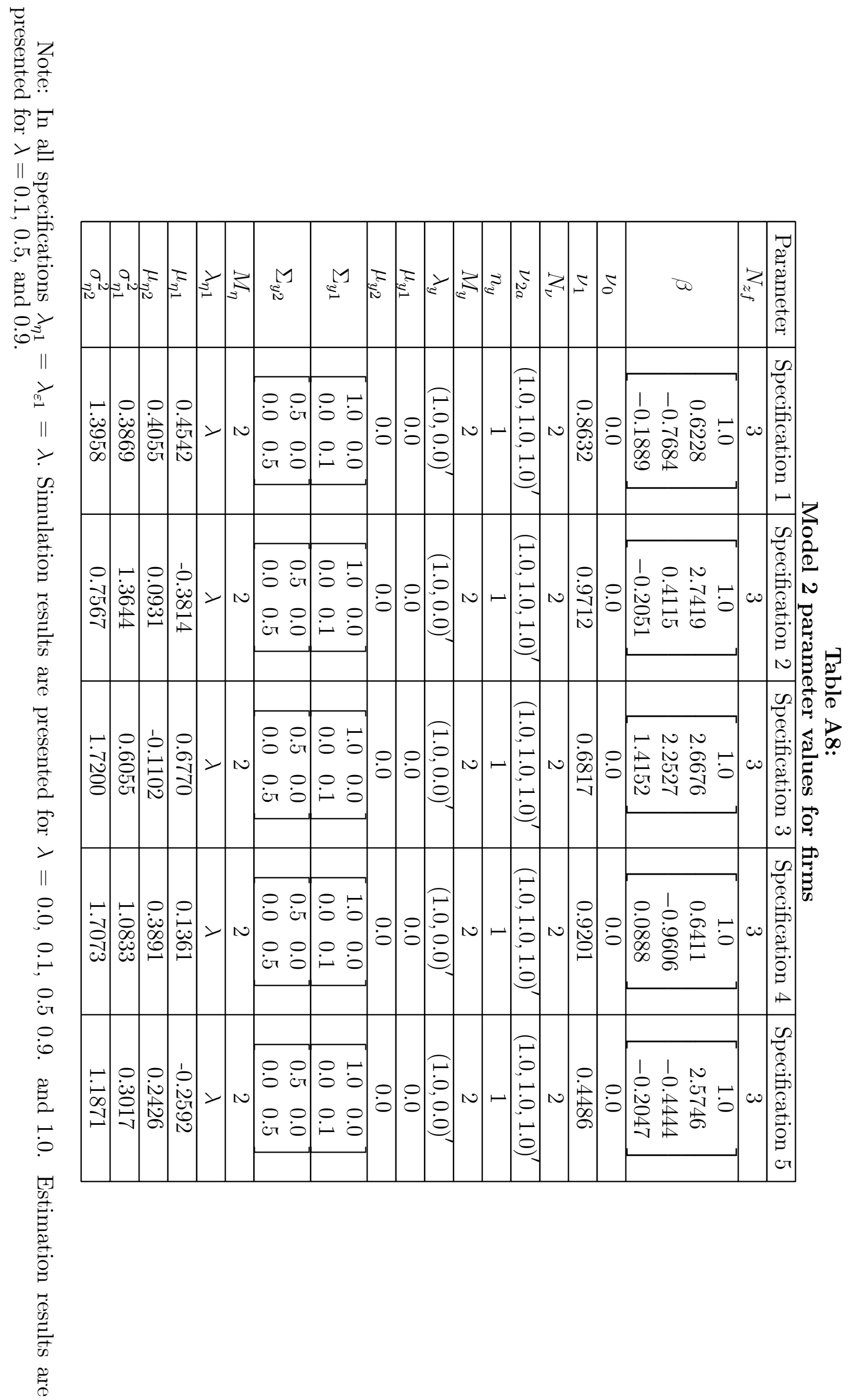




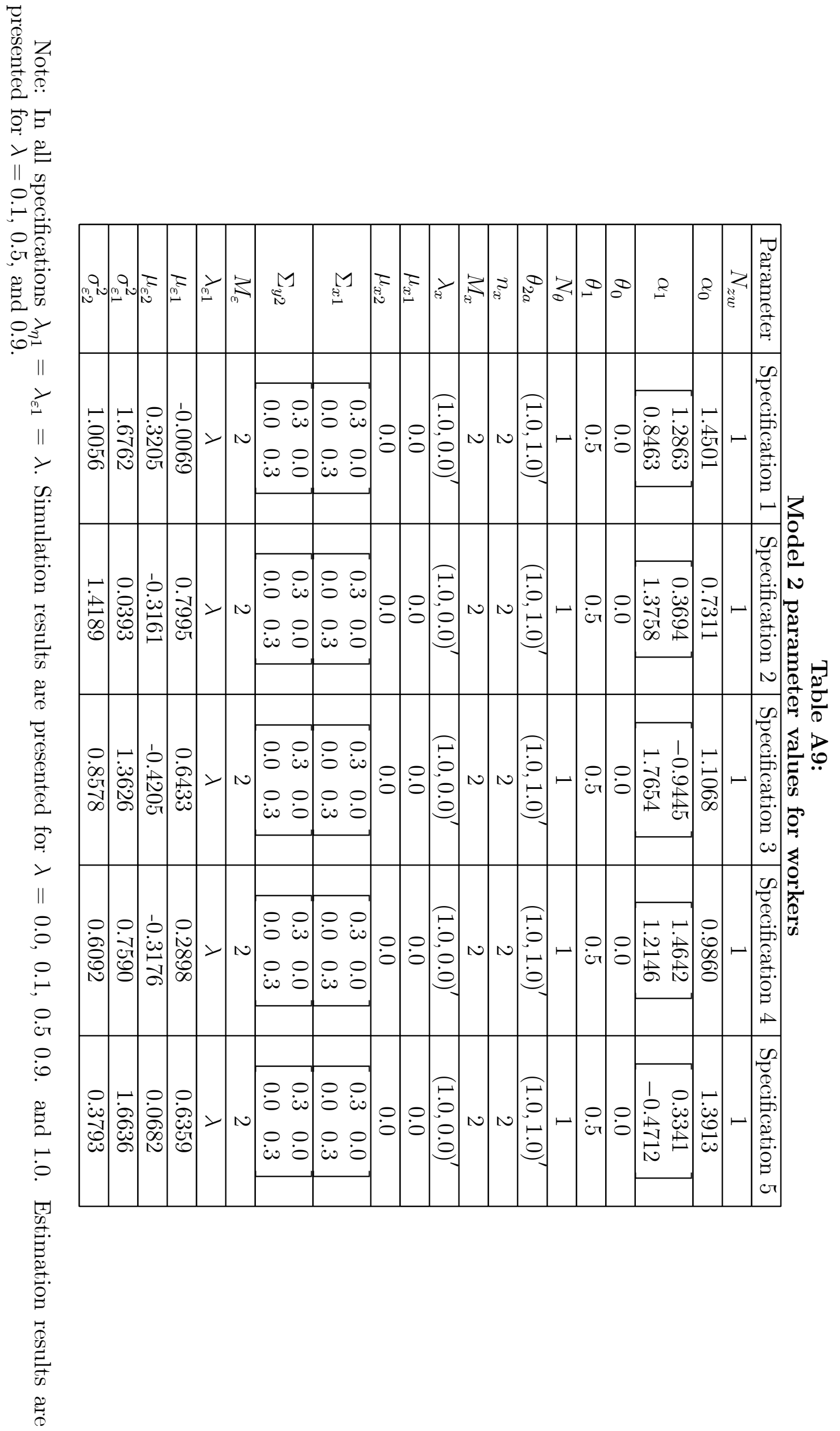


Table A10:

Model 2, Specification 1 parameter estimates

\begin{tabular}{|c|c|c|}
\multicolumn{3}{|c|}{$\lambda=0.5$} \\
\hline & True Value & ML \\
\hline$\beta_{1}$ & 0.623 & 0.637 \\
& & $(0.243)$ \\
\hline$\beta_{2}$ & -0.768 & -0.767 \\
& & $(0.0902)$ \\
\hline$\beta_{3}$ & -0.189 & -0.192 \\
& & $(0.0231)$ \\
\hline$\nu_{c 1}$ & 1.86 & 1.86 \\
& & $(0.256)$ \\
\hline$\nu_{c 2}$ & 1.00 & 0.992 \\
& & $(0.113)$ \\
\hline$\nu_{c 3}$ & 1.00 & 1.00 \\
& & $(0.0978)$ \\
\hline$\nu_{c 4}$ & 0.500 & 0.501 \\
& & $(0.0426)$ \\
\hline$\nu_{c 5}$ & 0.200 & 0.203 \\
& & $(0.0202)$ \\
\hline
\end{tabular}

Note: $\beta_{i}, i=1, . ., 3$ and $\nu_{c j}, j=1, \ldots, 5$ are the subset of structural parameters of firm technologies in Model 2 that are identified. $\beta_{0}$, which equals 1.0 in all specifications is not identified. The parameters $\nu_{c j}$ satisfy $\sum_{j=1}^{5} \nu_{c j} y^{j-1}=\nu_{1}+\left(\sum_{i=0}^{2} \nu_{2 a i} y^{i}\right)^{2} \cdot 100$ independent samples each of size 1000 were generated using the parameter values in Tables A8 and A9. In all specifications, $\lambda_{\eta 1}=\lambda_{\varepsilon 1}=0.5$. Column 1 reports the true values of the parameters used to simulate the data. 100 independent estimates were obtained using the maximum likelihood technique described in section 5.1. Column 2, labeled "ML", reports the averages and standard errors of these estimates. 
Table A11:

Model 2, Specification 2 parameter estimates

\begin{tabular}{|c|c|c|}
\hline & True Value & ML \\
\hline$\beta_{1}$ & 2.74 & 2.75 \\
& & $(0.0744)$ \\
\hline$\beta_{2}$ & 0.412 & 0.408 \\
& & $(0.0629)$ \\
\hline$\beta_{3}$ & -0.205 & -0.210 \\
& & $(0.0570)$ \\
\hline$\nu_{1}$ & 1.97 & 1.98 \\
& & $(0.246)$ \\
\hline$\nu_{2}$ & 1.00 & 1.00 \\
& & $(0.131)$ \\
\hline$\nu_{3}$ & 1.00 & 1.00 \\
& & $(0.123)$ \\
\hline$\nu_{4}$ & 0.500 & 0.504 \\
& & $(0.0480)$ \\
\hline$\nu_{5}$ & 0.200 & 0.202 \\
& & $(0.0175)$ \\
\hline
\end{tabular}

Note: $\beta_{i}, i=1, . ., 3$ and $\nu_{c j}, j=1, \ldots, 5$ are the subset of structural parameters of firm technologies in Model 2 that are identified. $\beta_{0}$, which equals 1.0 in all specifications is not identified. The parameters $\nu_{c j}$ satisfy $\sum_{j=1}^{5} \nu_{c j} y^{j-1}=\nu_{1}+\left(\sum_{i=0}^{2} \nu_{2 a i} y^{i}\right)^{2} \cdot 100$ independent samples each of size 1000 were generated using the parameter values in Tables A8 and A9. In all specifications, $\lambda_{\eta 1}=\lambda_{\varepsilon 1}=0.5$. Column 1 reports the true values of the parameters used to simulate the data. 100 independent estimates were obtained using the maximum likelihood technique described in section 5.1. Column 2, labeled "ML", reports the averages and standard errors of these estimates. 
Table A12:

Model 2, Specification 3 parameter estimates

\begin{tabular}{|c|c|c|}
\multicolumn{3}{|c|}{$\lambda=0.5$} \\
\hline & True Value & ML \\
\hline$\beta_{1}$ & 2.67 & $\begin{array}{c}2.67 \\
(0.200)\end{array}$ \\
\hline$\beta_{2}$ & 2.25 & $\begin{array}{c}2.27 \\
(0.206)\end{array}$ \\
\hline$\beta_{3}$ & 1.42 & 1.40 \\
& & $(0.120)$ \\
\hline$\nu_{1}$ & 1.68 & 1.69 \\
& & $(0.275)$ \\
\hline$\nu_{2}$ & 1.00 & 1.01 \\
& & $(0.146)$ \\
\hline$\nu_{3}$ & 1.00 & 1.01 \\
& & $(0.145)$ \\
\hline$\nu_{4}$ & 0.500 & 0.507 \\
& & $(0.0593)$ \\
\hline$\nu_{5}$ & 0.200 & 0.202 \\
& & $(0.0225)$ \\
\hline
\end{tabular}

Note: $\beta_{i}, i=1, . ., 3$ and $\nu_{c j}, j=1, \ldots, 5$ are the subset of structural parameters of firm technologies in Model 2 that are identified. $\beta_{0}$, which equals 1.0 in all specifications is not identified. The parameters $\nu_{c j}$ satisfy $\sum_{j=1}^{5} \nu_{c j} y^{j-1}=\nu_{1}+\left(\sum_{i=0}^{2} \nu_{2 a i} y^{i}\right)^{2} \cdot 100$ independent samples each of size 1000 were generated using the parameter values in Tables A8 and A9. In all specifications, $\lambda_{\eta 1}=\lambda_{\varepsilon 1}=0.5$. Column 1 reports the true values of the parameters used to simulate the data. 100 independent estimates were obtained using the maximum likelihood technique described in section 5.1. Column 2, labeled "ML", reports the averages and standard errors of these estimates. 
Table A13:

Model 2, Specification 4 parameter estimates

\begin{tabular}{|c|c|c|}
\hline & True Value & ML \\
\hline$\beta_{1}$ & 0.641 & 0.627 \\
& & $(0.337)$ \\
\hline$\beta_{2}$ & -0.961 & -0.984 \\
& & $(0.216)$ \\
\hline$\beta_{3}$ & 0.0888 & 0.0781 \\
& & $(0.0651)$ \\
\hline$\nu_{1}$ & 1.92 & 1.95 \\
& & $(0.330)$ \\
\hline$\nu_{2}$ & 1.00 & 1.00 \\
& & $(0.167)$ \\
\hline$\nu_{3}$ & 1.00 & 1.01 \\
& & $(0.151)$ \\
\hline$\nu_{4}$ & 0.500 & 0.509 \\
& & $(0.0636)$ \\
\hline$\nu_{5}$ & 0.200 & 0.204 \\
& & $(0.0259)$ \\
\hline
\end{tabular}

Note: $\beta_{i}, i=1, . ., 3$ and $\nu_{c j}, j=1, \ldots, 5$ are the subset of structural parameters of firm technologies in Model 2 that are identified. $\beta_{0}$, which equals 1.0 in all specifications is not identified. The parameters $\nu_{c j}$ satisfy $\sum_{j=1}^{5} \nu_{c j} y^{j-1}=\nu_{1}+\left(\sum_{i=0}^{2} \nu_{2 a i} y^{i}\right)^{2} \cdot 100$ independent samples each of size 1000 were generated using the parameter values in Tables A8 and A9. In all specifications, $\lambda_{\eta 1}=\lambda_{\varepsilon 1}=0.5$. Column 1 reports the true values of the parameters used to simulate the data. 100 independent estimates were obtained using the maximum likelihood technique described in section 5.1. Column 2, labeled "ML", reports the averages and standard errors of these estimates. 
Table A14:

Model 2 Specification 5 parameter estimates

$\lambda=0.5$

\begin{tabular}{|c|c|c|}
\hline & True Value & ML \\
\hline$\beta_{1}$ & 2.5746 & 2.58 \\
& & $(0.103)$ \\
\hline$\beta_{2}$ & -0.4444 & -0.440 \\
& & $(0.0251)$ \\
\hline$\beta_{3}$ & -0.2047 & -0.206 \\
& & $(0.0072)$ \\
\hline$\nu_{1}$ & 1.4486 & 1.44 \\
& & $(0.193)$ \\
\hline$\nu_{2}$ & 1.00 & 0.988 \\
& & $(0.141)$ \\
\hline$\nu_{3}$ & 1.00 & 1.01 \\
& & $(0.111)$ \\
\hline$\nu_{4}$ & 0.500 & 0.512 \\
& & $(0.0513)$ \\
\hline$\nu_{5}$ & 0.200 & 0.205 \\
& & $(0.0203)$ \\
\hline
\end{tabular}

Note: $\beta_{i}, i=1, . ., 3$ and $\nu_{c j}, j=1, \ldots, 5$ are the subset of structural parameters of firm technologies in Model 2 that are identified. $\beta_{0}$, which equals 1.0 in all specifications is not identified. The parameters $\nu_{c j}$ satisfy $\sum_{j=1}^{5} \nu_{c j} y^{j-1}=\nu_{1}+\left(\sum_{i=0}^{2} \nu_{2 a i} y^{i}\right)^{2} \cdot 100$ independent samples each of size 1000 were generated using the parameter values in Tables A8 and A9. In all specifications, $\lambda_{\eta 1}=\lambda_{\varepsilon 1}=0.5$. Column 1 reports the true values of the parameters used to simulate the data. 100 independent estimates were obtained using the maximum likelihood technique described in section 5.1. Column 2, labeled "ML", reports the averages and standard errors of these estimates. 
Table A15:

Model 2 Specification 1 parameter estimates

\begin{tabular}{|c|c|c|}
\hline \\
\hline & True Value & $\mathrm{ML}$ \\
\hline$\beta_{1}$ & 0.623 & $\begin{array}{c}0.619 \\
(0.291)\end{array}$ \\
\hline$\beta_{2}$ & -0.768 & $\begin{array}{c}-0.776 \\
(0.115)\end{array}$ \\
\hline$\beta_{3}$ & -0.189 & $\begin{array}{c}0.194 \\
(0.0302)\end{array}$ \\
\hline$\nu_{1}$ & 1.86 & $\begin{array}{c}1.88 \\
(0.283)\end{array}$ \\
\hline$\nu_{2}$ & 1.00 & $\begin{array}{c}1.00 \\
(0.149)\end{array}$ \\
\hline$\nu_{3}$ & 1.00 & $\begin{array}{c}1.01 \\
(0.132)\end{array}$ \\
\hline$\nu_{4}$ & 0.500 & $\begin{array}{c}0.505 \\
(0.0551)\end{array}$ \\
\hline$\nu_{5}$ & 0.200 & $\begin{array}{c}0.204 \\
(0.0252)\end{array}$ \\
\hline
\end{tabular}

Note: $\beta_{i}, i=1, . ., 3$ and $\nu_{c j}, j=1, \ldots, 5$ are the subset of structural parameters of firm technologies in Model 2 that are identified. $\beta_{0}$, which equals 1.0 in all specifications is not identified. The parameters $\nu_{c j}$ satisfy $\sum_{j=1}^{5} \nu_{c j} y^{j-1}=\nu_{1}+\left(\sum_{i=0}^{2} \nu_{2 a i} y^{i}\right)^{2} \cdot 100$ independent samples each of size 1000 were generated using the parameter values in Tables A8 and A9. In all specifications, $\lambda_{\eta 1}=\lambda_{\varepsilon 1}=0.9$. Column 1 reports the true values of the parameters used to simulate the data. 100 independent estimates were obtained using the maximum likelihood technique described in section 5.1. Column 2, labeled "ML", reports the averages and standard errors of these estimates. 
Table A16:

Model 2, Specification 2 parameter estimates

\begin{tabular}{|c|c|c|}
\hline & True Value & ML \\
\hline$\beta_{1}$ & 2.74 & 2.75 \\
& & $(0.0705)$ \\
\hline$\beta_{2}$ & 0.412 & 0.403 \\
& & $(0.0553)$ \\
\hline$\beta_{3}$ & -0.205 & -0.216 \\
& & $(0.0506)$ \\
\hline$\nu_{1}$ & 1.97 & 2.00 \\
& & $(0.228)$ \\
\hline$\nu_{2}$ & 1.00 & 1.01 \\
& & $(0.122)$ \\
\hline$\nu_{3}$ & 1.00 & 1.02 \\
& & $(0.108)$ \\
\hline$\nu_{4}$ & 0.500 & 0.510 \\
& & $(0.0422)$ \\
\hline$\nu_{5}$ & 0.200 & 0.203 \\
& & $(0.0154)$ \\
\hline
\end{tabular}

Note: $\beta_{i}, i=1, . ., 3$ and $\nu_{c j}, j=1, \ldots, 5$ are the subset of structural parameters of firm technologies in Model 2 that are identified. $\beta_{0}$, which equals 1.0 in all specifications is not identified. The parameters $\nu_{c j}$ satisfy $\sum_{j=1}^{5} \nu_{c j} y^{j-1}=\nu_{1}+\left(\sum_{i=0}^{2} \nu_{2 a i} y^{i}\right)^{2} \cdot 100$ independent samples each of size 1000 were generated using the parameter values in Tables A8 and A9. In all specifications, $\lambda_{\eta 1}=\lambda_{\varepsilon 1}=0.9$. Column 1 reports the true values of the parameters used to simulate the data. 100 independent estimates were obtained using the maximum likelihood technique described in section 5.1. Column 2, labeled "ML", reports the averages and standard errors of these estimates. 
Table A17:

Model 2 Specification 3 parameter estimates

\begin{tabular}{|c|c|c|}
\multicolumn{3}{c}{$\lambda=0.9$} \\
\hline & True Value & ML \\
\hline$\beta_{1}$ & 2.67 & $\begin{array}{c}2.66 \\
(0.218)\end{array}$ \\
\hline$\beta_{2}$ & 2.25 & $\begin{array}{c}2.27 \\
(0.230)\end{array}$ \\
\hline$\beta_{3}$ & 1.42 & $\begin{array}{c}1.40 \\
(0.149)\end{array}$ \\
\hline$\nu_{1}$ & 1.68 & 1.72 \\
& & $(0.322)$ \\
\hline$\nu_{2}$ & 1.00 & 1.02 \\
& & $(0.191)$ \\
\hline$\nu_{3}$ & 1.00 & $\begin{array}{c}1.02 \\
(0.183)\end{array}$ \\
\hline$\nu_{4}$ & 0.500 & 0.512 \\
& & $(0.0766)$ \\
\hline$\nu_{5}$ & 0.200 & 0.203 \\
& & $(0.0284)$ \\
\hline
\end{tabular}

Note: $\beta_{i}, i=1, . ., 3$ and $\nu_{c j}, j=1, \ldots, 5$ are the subset of structural parameters of firm technologies in Model 2 that are identified. $\beta_{0}$, which equals 1.0 in all specifications is not identified. The parameters $\nu_{c j}$ satisfy $\sum_{j=1}^{5} \nu_{c j} y^{j-1}=\nu_{1}+\left(\sum_{i=0}^{2} \nu_{2 a i} y^{i}\right)^{2} \cdot 100$ independent samples each of size 1000 were generated using the parameter values in Tables A8 and A9. In all specifications, $\lambda_{\eta 1}=\lambda_{\varepsilon 1}=0.9$. Column 1 reports the true values of the parameters used to simulate the data. 100 independent estimates were obtained using the maximum likelihood technique described in section 5.1. Column 2, labeled "ML", reports the averages and standard errors of these estimates. 
Table A18:

Model 2, Specification 4 parameter estimates

\begin{tabular}{|c|c|c|}
\hline & True Value & ML \\
\hline$\beta_{1}$ & 0.641 & 0.640 \\
& & $(0.405)$ \\
\hline$\beta_{2}$ & -0.961 & -0.980 \\
& & $(0.266)$ \\
\hline$\beta_{3}$ & 0.0888 & 0.079 \\
& & $(0.0809)$ \\
\hline$\nu_{1}$ & 1.92 & 1.933 \\
& & $(0.366)$ \\
\hline$\nu_{2}$ & 1.00 & 0.998 \\
& & $(0.196)$ \\
\hline$\nu_{3}$ & 1.00 & 1.01 \\
& & $(0.190)$ \\
\hline$\nu_{4}$ & 0.500 & 0.508 \\
& & $(0.0785)$ \\
\hline$\nu_{5}$ & 0.200 & 0.204 \\
& & $(0.0316)$ \\
\hline
\end{tabular}

Note: $\beta_{i}, i=1, . ., 3$ and $\nu_{c j}, j=1, \ldots, 5$ are the subset of structural parameters of firm technologies in Model 2 that are identified. $\beta_{0}$, which equals 1.0 in all specifications is not identified. The parameters $\nu_{c j}$ satisfy $\sum_{j=1}^{5} \nu_{c j} y^{j-1}=\nu_{1}+\left(\sum_{i=0}^{2} \nu_{2 a i} y^{i}\right)^{2} \cdot 100$ independent samples each of size 1000 were generated using the parameter values in Tables A8 and A9. In all specifications, $\lambda_{\eta 1}=\lambda_{\varepsilon 1}=0.9$. Column 1 reports the true values of the parameters used to simulate the data. 100 independent estimates were obtained using the maximum likelihood technique described in section 5.1. Column 2, labeled "ML", reports the averages and standard errors of these estimates. 
Table A19:

Model 2, Specification 5 parameter estimates

\begin{tabular}{|c|c|c|}
\multicolumn{3}{|c|}{$\lambda=0.9$} \\
\hline & True Value & ML \\
\hline$\beta_{1}$ & 2.57 & 2.58 \\
& & $(0.137)$ \\
\hline$\beta_{2}$ & -0.444 & -0.440 \\
& & $(0.0271)$ \\
\hline$\beta_{3}$ & -0.205 & -0.206 \\
& & $(0.0101)$ \\
\hline$\nu_{1}$ & 1.45 & 1.44 \\
& & $(0.217)$ \\
\hline$\nu_{2}$ & 1.00 & 0.987 \\
& & $(0.165)$ \\
\hline$\nu_{3}$ & 1.00 & 1.01 \\
& & $(0.152)$ \\
\hline$\nu_{4}$ & 0.500 & 0.514 \\
& & $(0.0746)$ \\
\hline$\nu_{5}$ & 0.200 & 0.205 \\
& & $(0.0279)$ \\
\hline
\end{tabular}

Note: $\beta_{i}, i=1, . ., 3$ and $\nu_{c j}, j=1, \ldots, 5$ are the subset of structural parameters of firm technologies in Model 2 that are identified. $\beta_{0}$, which equals 1.0 in all specifications is not identified. The parameters $\nu_{c j}$ satisfy $\sum_{j=1}^{5} \nu_{c j} y^{j-1}=\nu_{1}+\left(\sum_{i=0}^{2} \nu_{2 a i} y^{i}\right)^{2} \cdot 100$ independent samples each of size 1000 were generated using the parameter values in Tables A8 and A9. In all specifications, $\lambda_{\eta 1}=\lambda_{\varepsilon 1}=0.9$. Column 1 reports the true values of the parameters used to simulate the data. 100 independent estimates were obtained using the maximum likelihood technique described in section 5.1. Column 2, labeled "ML", reports the averages and standard errors of these estimates. 


\section{References}

[1] Armstrong, M. (1996). "Multiproduct Nonlinear Pricing." Econometrica, 64(1): 51-75.

[2] Banzhaf, H., H. Sieg, V. K. Smith, and R. Walsh (2000). "Estimating the General Equilibrium Benefits of Large Changes in Spatially Delineated Public Goods," NBER Working Paper No. 7744.

[3] Bayer, P. (2000). "Tiebout Sorting and Discrete Choices: A New Explanation for Socioeconomic Differences in the Consumption of School Quality," mimeo, Yale University.

[4] Bresnahan, T. and R. Gordon (1997). The Economics of New Goods, Chicago: University of Chicago Press.

[5] Brown, J. and H. Rosen (1982). "On the Estimation of Structural Hedonic Price Models," Econometrica, 50: 765-769.

[6] Ekeland, I., J. Heckman and L. Nesheim (2002). "Identification and Estimation of Hedonic Models," forthcoming Journal of Political Economy.

[7] Epple, D. (1987). "Hedonic Prices and Implicit Markets: Estimating Demand and Supply Functions for Differentiated Products," Journal of Political Economy, 95: 59-80.

[8] Epple, D. and H. Sieg (1999). "Estimating Equilibrium Models of Local Jurisdictions," Journal of Political Economy, 107: 645-681.

[9] Gretsky, N., J. Ostroy and W. R. Zame (1999). "Perfect Competition in the Continuous Assignment Model," Journal of Economic Theory, 88: 60-118.

[10] Heckman J., Matzkin R., and Nesheim, L. (2002), "Non-parametric estimation of nonadditive hedonic models," unpublished working paper.

[11] Horowitz, J. (1996). "Semiparametric Estimation of a Regression Model with an Unknown Transformation of the Dependent Variable," Econometrica, 64: 103-137.

[12] Kahn, S. and K. Lang (1988). "Efficient Estimation of Structural Hedonic Systems," International Economic Review, 29: 157-166.

[13] Kniesner, T. and J. Leeth (1988). "Simulating Hedonic Labor Market Models: Computational Issues and Policy Applications," International Economic Review, 29(4): 755-789. 
[14] Kniesner, T. and J. Leeth (1995). Simulating Workplace Safety Policy, Boston: Kluwer Academic.

[15] Lancaster, K. (1966). "A New Approach to Consumer Theory," Journal of Political Economy, 74(2): 132-157.

[16] Lancaster, K. (1975). "Socially Optimal Product Differentiation," American Economic Review, 65(4): 567-585.

[17] Matzkin, R. (1999). "Nonparametric Estimation of Nonadditive Random Functions," 1st version, working paper, Northwestern University.

[18] Matzkin, R. (2003). "Nonparametric Estimation of Nonadditive Random Functions," forthcoming, Econometrica.

[19] Matzkin, R.L. (2002). "Estimation of Nonadditive Functions Using Functional Restrictions," working paper, Northwestern University.

[20] Nesheim, L. (2001). "Equilibrium Sorting of Heterogeneous Consumers Across Locations: Theory and Empirical Implications," Ph.D. Thesis, University of Chicago.

[21] Rochet, J.-C., and L. Stole (2001). "The Economics of Multidimensional Screening," working paper, The University of Chicago.

[22] Rosen, S. (1974). "Hedonic Prices and Implicit Markets: Product Differentiation in Pure Competition," Journal of Political Economy, 82: $34-55$.

[23] Sattinger, M. (1975). "Comparative Advantage and the Distributions of Earnings and Abilities," Econometrica, 43(3): 455-468.

[24] Sattinger, M. (1980). Capital and the Distribution of Labor Earnings, New York: North Holland.

[25] Sattinger, M. (1993). "Assignment Models of the Distribution of Earnings," Journal of Economic Literature, 31(2): 831-880.

[26] Smith, V. K., and J. C. Huang (1995). "Can Markets Value Air Quality: A Meta-Analysis of Hedonic Property Value Models," Journal of Political Economy, 103(1):209-227.

[27] Teulings, C. (1995). "The Wage Distribution in a Model of the Assignment of Jobs to Skills," Journal of Political Economy, 103(2): 280-315.

[28] Teulings, C. and T. van Rens (2002). "Education Growth and Income Inequality," Tinbergen Institute Discussion Paper TI 2002-001/3. 
[29] Thaler, R. and S. Rosen (1975). "The Value of Saving A Life: Evidence From The Labor Market," in Nester Terleckyj (ed.), Household Production and Consumption, National Bureau of Economic Research.

[30] Tinbergen, J. (1956). "On The Theory of Income Distribution," Weltwirtschaftliches Archiv, 77: 155-73.

[31] Triplett, J. (2000). Handbook on Quality Adjustment of Price Indexes for Information and Communication Technology Products, Paris: OECD.

[32] Wilson, R. (1993). Nonlinear Pricing, Oxford: Oxford University Press.

[33] Wooders, M. (1994). "Equivalence of Games and Markets," Econometrica, 62(5): 1141-1160. 


\section{IZA Discussion Papers}

\begin{tabular}{|c|c|c|c|c|}
\hline No. & Author(s) & Title & Area & Date \\
\hline 829 & $\begin{array}{l}\text { J. J. Heckman } \\
\text { X. Li }\end{array}$ & $\begin{array}{l}\text { Selection Bias, Comparative Advantage and } \\
\text { Heterogeneous Returns to Education: Evidence } \\
\text { from China in } 2000\end{array}$ & 6 & $07 / 03$ \\
\hline 830 & T. J. Hatton & $\begin{array}{l}\text { Emigration from the UK, } 1870-1913 \text { and } 1950- \\
1998\end{array}$ & 1 & $07 / 03$ \\
\hline 831 & $\begin{array}{l}\text { J. H. Abbring } \\
\text { G. J. van den Berg }\end{array}$ & $\begin{array}{l}\text { Analyzing the Effect of Dynamically Assigned } \\
\text { Treatments Using Duration Models, Binary } \\
\text { Treatment Models, and Panel Data Models }\end{array}$ & 6 & $07 / 03$ \\
\hline 832 & P.-C. Michaud & Joint Labour Supply Dynamics of Older Couples & 3 & $07 / 03$ \\
\hline 833 & H. Gersbach & $\begin{array}{l}\text { Structural Reforms and the Macroeconomy: The } \\
\text { Role of General Equilibrium Effects }\end{array}$ & 2 & $07 / 03$ \\
\hline 834 & $\begin{array}{l}\text { T. Boeri } \\
\text { J. I. Conde-Ruiz } \\
\text { V. Galasso }\end{array}$ & $\begin{array}{l}\text { Protecting Against Labour Market Risk: } \\
\text { Employment Protection or Unemployment } \\
\text { Benefits? }\end{array}$ & 3 & $07 / 03$ \\
\hline 835 & $\begin{array}{l}\text { G. Joseph } \\
\text { O. Pierrard } \\
\text { H. R. Sneessens }\end{array}$ & $\begin{array}{l}\text { Job Turnover, Unemployment and Labor Market } \\
\text { Institutions }\end{array}$ & 3 & $07 / 03$ \\
\hline 836 & M.-S. Yun & $\begin{array}{l}\text { A Simple Solution to the Identification Problem } \\
\text { in Detailed Wage Decompositions }\end{array}$ & 3 & $07 / 03$ \\
\hline 837 & W. Schnedler & $\begin{array}{l}\text { What You Always Wanted to Know About } \\
\text { Censoring But Never Dared to Ask - Parameter } \\
\text { Estimation for Censored Random Vectors }\end{array}$ & 6 & $07 / 03$ \\
\hline 838 & $\begin{array}{l}\text { S. Girma } \\
\text { H. Görg } \\
\text { E. Strobl }\end{array}$ & $\begin{array}{l}\text { Government Grants, Plant Survival and } \\
\text { Employment Growth: A Micro-Econometric } \\
\text { Analysis }\end{array}$ & 6 & $07 / 03$ \\
\hline 839 & $\begin{array}{l}\text { A. Bassanini } \\
\text { G. Brunello }\end{array}$ & $\begin{array}{l}\text { Is Training More Frequent When Wage } \\
\text { Compression is Higher? Evidence from the } \\
\text { European Community Household Panel }\end{array}$ & 6 & 08/03 \\
\hline 840 & $\begin{array}{l}\text { E. Koskela } \\
\text { R. Stenbacka }\end{array}$ & $\begin{array}{l}\text { Equilibrium Unemployment Under Negotiated } \\
\text { Profit Sharing }\end{array}$ & 1 & 08/03 \\
\hline 841 & $\begin{array}{l}\text { B. H. Baltagi } \\
\text { D. P. Rich }\end{array}$ & $\begin{array}{l}\text { Skill-Biased Technical Change in U.S. } \\
\text { Manufacturing: A General Index Approach }\end{array}$ & 1 & 08/03 \\
\hline 842 & $\begin{array}{l}\text { M. Svarer } \\
\text { M. Rosholm } \\
\text { J. R. Munch }\end{array}$ & Rent Control and Unemployment Duration & 3 & $08 / 03$ \\
\hline 843 & $\begin{array}{l}\text { J. J. Heckman } \\
\text { R. Matzkin } \\
\text { L. P. Nesheim }\end{array}$ & Simulation and Estimation of Hedonic Models & 6 & 08/03 \\
\hline
\end{tabular}

An updated list of IZA Discussion Papers is available on the center's homepage www.iza.org. 